\title{
Radiation and dissipation of internal waves generated by geostrophic motions impinging on small-scale topography \\ by \\ Maxim Nikurashin
}

M.S., Moscow Institute of Physics and Technology, Russia, 2002

Submitted in partial fulfillment of the requirements for the degree of

Doctor of Philosophy

at the

MASSACHUSETTS INSTITUTE OF TECHNOLOGY

and the

WOODS HOLE OCEANOGRAPHIC INSTITUTION

February 2009

(C) Maxim Nikurashin, 2009

The author hereby grants to MIT and to WHOI permission to reproduce and distribute publicly paper and electronic copies of this thesis document in whole or in part.

Author

Joint Program in Physical Oceanography Massachusetts Institute of Technology Woods Hole Oceanographic Institution September 26, 2008

Certified by

Raffaele Ferrari Associate Professor

Thesis Supervisor

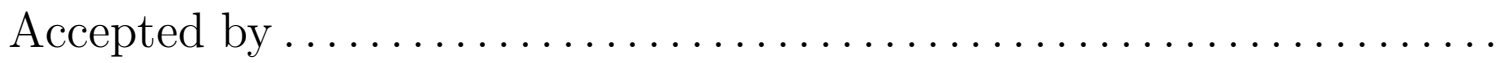

Maria T. Zuber

E.A. Griswold Professor of Geophysics

Head, Department of Earth, Atmospheric and Planetary Sciences 


\title{
Radiation and dissipation of internal waves generated by geostrophic motions impinging on small-scale topography
}

\author{
by \\ Maxim Nikurashin \\ Submitted to the Joint Program in Physical Oceanography - Massachusetts \\ Institute of Technology / Woods Hole Oceanographic Institution \\ on September 26, 2008, in partial fulfillment of the \\ requirements for the degree of \\ Doctor of Philosophy
}

\begin{abstract}
Observations and inverse models suggest that small-scale turbulent mixing is enhanced in the Southern Ocean in regions above rough topography. The enhancement extends $1 \mathrm{~km}$ above the topography suggesting that mixing is supported by breaking of gravity waves radiated from the ocean bottom. In other regions, gravity wave radiation by bottom topography has been primarily associated with the barotropic tide. In this study, we explore the alternative hypothesis that the enhanced mixing in the Southern Ocean is sustained by internal waves generated by geostrophic motions flowing over bottom topography. Weakly-nonlinear theory is used to describe the internal wave generation and the feedback of the waves on the zonally averaged flow. A major finding is that the waves generated at the ocean bottom at finite inverse Froude numbers drive vigorous inertial oscillations. The wave radiation and dissipation at equilibrium is therefore the result of both geostrophic flow and inertial oscillations and differs substantially from the classical lee wave problem. The theoretical predictions are tested versus two-dimensional and three-dimensional high resolution numerical simulations with parameters representative of the Drake Passage region. Theory and fully nonlinear numerical simulations are used to estimate internal wave radiation from LADCP, CTD and topography data from two regions in the Southern Ocean: Drake Passage and the Southeast Pacific. The results show that radiation and dissipation of internal waves generated by geostrophic motions reproduce the magnitude and distribution of dissipation measured in the region.
\end{abstract}

Thesis Supervisor: Raffaele Ferrari

Title: Associate Professor 


\section{Acknowledgments}

I am deeply grateful to many people who contributed to this thesis. First and foremost, I would like to thank my advisor, Raffaele Ferrari, for his enthusiasm, help, and guidance over the course of my doctoral study. This work would not have been possible without his endless patience and continuous support.

I am grateful to my dissertation committee members, Glenn Flierl, Jim Ledwell, John Marshall, Alan Plumb, and Kurt Polzin for their participation, helpful input, and useful criticism. I wish to thank Alberto Naveira-Garabato for his help with the observations and useful suggestions, and Jean-Michel Campin for his assistance with the MITgcm. I also wish to thank Carl Wunsch for chairing my defense as well as for his expert advice.

The Academic Programs Office at WHOI and the Education Office at MIT have been very helpful. I would especially like to thank Julia Westwater, Marsha Gomes, Ronni Schwartz, and Carol Sprague.

My friends, classmates, and officemates have been always a source of help, information and fun. I thank them for making my graduate student career an enjoyable experience.

A special thanks to my family, my wonderful wife and best friend Tatiana for her love, support, and encouragement, and my 15-month-old son Denis for making me

proud. Finally, I dedicate this thesis to my parents, Anatoly and Ludmila. I can never thank them enough for all that they have given me. 


\section{Contents}

1 Introduction $\quad 17$

1.1 Observational estimates of abyssal mixing . . . . . . . . . . . . 18

1.2 Major contributors to mixing . . . . . . . . . . . . . 21

1.2.1 Internal tides . . . . . . . . . . . . . . . . . . . . . . . 21

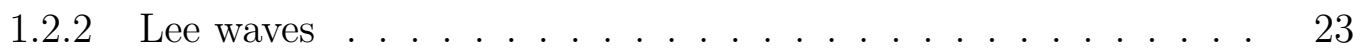

1.3 Large-scale flow - topography interaction . . . . . . . . . . . . . . 27

1.3.1 Steady flow . . . . . . . . . . . . . . . 28

1.3.2 Oscillatory flow . . . . . . . . . . . . . . . 30

1.3.3 Abyssal flows in the ocean . . . . . . . . . . . . . 31

1.4 Overview of the thesis . . . . . . . . . . . . . . . . . . 32

$2 \quad 2-D$ Theory and Simulations $\quad 37$

2.1 Introduction . . . . . . . . . . . . . . . 37

2.2 Simulations . . . . . . . . . . . . . . . . . . 40

2.2.1 Experiment setup . . . . . . . . . . . . 41

2.2.2 Simulation results . . . . . . . . . . . . . . . . . . 42

2.3 Dynamical regimes . . . . . . . . . . . . . . . . . . 44

2.4 Theory ........................... 46

2.4.1 Problem formulation . . . . . . . . . . . . . 48

2.4.2 $O\left(\epsilon^{0}\right)$ solution: mean flow . . . . . . . . . . . . . . 50

2.4.3 $O\left(\epsilon^{1}\right)$ solution: wave radiation . . . . . . . . . . 50

2.4.4 $O\left(\epsilon^{3}\right)$ solution: feedback of waves on mean flow . . . . . . 53

2.5 Results . . . . . . . . . . . . . . . . 57 
2.5.1 Inertial oscillations . . . . . . . . . . . . . 57

2.5.2 Wave radiation . . . . . . . . . . . . . . 59

2.5 .3 Energy pathways . . . . . . . . . . . . . . 61

2.5.4 Wave breaking and dissipation . . . . . . . . . 65

2.6 Conclusions . . . . . . . . . . . . . . . . . . . . . . 66

3 Application to the Southern Ocean $\quad 85$

3.1 Introduction . . . . . . . . . . . . . 85

3.2 Data . . . . . . . . . . . . . . . . . 87

3.2 .1 Mean flow characteristics . . . . . . . . . . 88

3.2 .2 Topography characteristics . . . . . . . . . . . . . . . . 89

3.3 Energy radiation theory $\ldots \ldots \ldots$. . . . . . . . . . . . 92

3.3 .1 Lee wave radiation $\ldots \ldots \ldots \ldots$

3.3 .2 Simplified theory . . . . . . . . . . . . . . . . . 94

3.4 Results . . . . . . . . . . . . . . . . . . . . 96

3.4.1 Effective topography spectrum . . . . . . . . . . . 96

3.4 .2 Inverse Froude number estimate . . . . . . . . . . . . 98

3.4.3 Energy radiation estimate . . . . . . . . . . . 100

3.5 Multichromatic topography simulations . . . . . . . . . . . . . 101

3.5.1 Experiment setup . . . . . . . . . . . . . . . . 102

3.5.2 Results . . . . . . . . . . . . . . . . . . . . 103

3.6 Conclusions . . . . . . . . . . . . . . . . . . . . . . . . . . . . . . . 104

4 3-D Theory and Simulations $\quad 129$

4.1 Introduction . . . . . . . . . . . . . . . . . . . . . . 129

4.2 Theory . . . . . . . . . . . . . . . . . . . . . 130

4.3 Experiment setup . . . . . . . . . . . . . . . . . . . . 133

4.4 Results . . . . . . . . . . . . . . . . . . . . . 134

4.4 .1 Inverse Froude number . . . . . . . . . . . . . . . . 134

4.4 .2 Inertial oscillations . . . . . . . . . . . . . . 135

4.4 .3 Wave radiation . . . . . . . . . . . . 136 
4.4.4 Wave breaking and dissipation . . . . . . . . . . . . . . 137

4.5 Conclusions . . . . . . . . . . . . . . . . . . . . 138

5 Summary and Conclusions $\quad 147$

5.1 Summary of the thesis . . . . . . . . . . . . . . . 147

5.2 Implications . . . . . . . . . . . . . . . . . . 150

5.2.1 Energetics and overturning circulation of the SO . . . . . . . 150

5.2.2 Geostrophic flows and abyssal mixing in the global ocean . . . 152

5.2.3 Geostrophic eddy energy budget . . . . . . . . . . . . 153

5.3 Future work . . . . . . . . . . . . . . . . 155

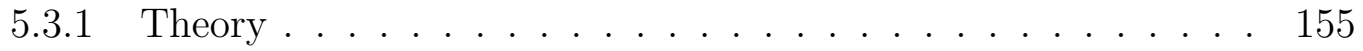

5.3 .2 Observations ....................... 156

A The equations of motion: scaling $\quad 159$

B The equations of motion: multiple scale expansion 161 


\section{List of Figures}

1-1 Bathymetry and depth-averaged current vectors along the I8S section (left panel). Vertical profile of $\mathrm{N}^{2}\left(\mathrm{~s}^{-2}\right)$ (thick lines) and $\kappa_{\rho}\left(\mathrm{m}^{2} \mathrm{~s}^{-1}\right)$ (thin lines) for two groups of twelve stations, one at $35^{\circ} \mathrm{S}$ and the other at $55^{\circ}$ S. From Polzin and Firing (1997). . . . . . . . . . . 33

1-2 Bathymetry of the Southern Ocean region around Drake Passage. CTD and LADCP stations are shown by colored diamonds. Open circles mark stations where only CTD data are available. The black lines indicate the northern and southern boundaries of the ACC. From NaveiraGarabato et al. (2004). Reprinted with permission from AAAS. . . .

1-3 Averaged profiles of $\mathrm{N}^{2}\left(\mathrm{~s}^{-2}\right), \epsilon\left(\mathrm{W} \mathrm{kg}^{-1}\right), \kappa_{\rho}\left(\mathrm{m}^{2} \mathrm{~s}^{-1}\right)$ for the Southeast Pacific (section A in Fig. 1-2) and the Drake Passage (section B in Fig. 1-2) regions. From Naveira-Garabato et al. (2004). Reprinted with permission from AAAS. . . . . . . . . . . . .

2-1 Snapshot of the wave zonal velocity $\left(\mathrm{m} \mathrm{s}^{-1}\right)$ from $F r^{-1}=0.1$ simulation. 70

2-2 Snapshot of the wave zonal velocity $\left(\mathrm{m} \mathrm{s}^{-1}\right)$ from $F r^{-1}=0.4$ simulation. 71

2-3 Time evolution of zonally averaged velocities $\left(\mathrm{m} \mathrm{s}^{-1}\right)$ from $\mathrm{Fr}^{-1}=$ 0.4 simulation: (upper panel) deviation from an externally prescribed $0.1 \mathrm{~m} \mathrm{~s}^{-1}$ zonal mean flow,(lower panel) meridional velocity component. 72 
2-4 Definition sketch illustrating interaction of a geostrophic flow $U_{G}$ and an inertial oscillation $\left(U_{I}, f\right)$ with periodic bottom topography $\left(k_{T}, h_{T}\right)$ in stably stratified fluid. Dashed gray lines are the phase lines of the waves and the dashed black line is the trajectory of a particle, illustrating its excursion during one period of oscillation. . . . . . . .

2-5 Sketch illustrating resonant forcing mechanism driving inertial oscillations. Straight and dashed vertical lines are the topography crests and troughs, respectively. Gray and white arrows show, respectively, the direction of inertial oscillation velocity vector and the direction of the wave momentum flux divergence. Circular (black) and elliptical (gray) trajectories indicate the direction of rotation for the inertial oscillation $(f>0)$ and flux divergence vectors, respectively. . . . . . . . . . . . 74

2-6 Evolution of the bottom value of the inertial oscillation amplitude (m $\mathrm{s}^{-1}$ ) from different simulations. . . . . . . . . . . . . . . . . 75

2-7 Evolution of the bottom value of the inertial oscillation amplitude normalized by its initial value, $\log \frac{U_{I}(t)}{U_{I}(0)}$, from the $F r^{-1}=0.4$ simulation (gray), resonant feedback theory prediction from (2.36) and (2.38) (solid black), and linear growth rate prediction (2.49) (dashed black). 76

2-8 Profiles of the vertical energy flux $\left(\mathrm{mW} \mathrm{m}^{-2}\right)$ from different simulations. 77

2-9 Energy conversion rate at the bottom as a function of $\mathrm{Fr}^{-2}$ : diagnosed from simulations (black squares), predicted from linear lee wave theory (gray line), and predicted from linear theory for inertial harmonics (black line). . . . . . . . . . . . . . . . . .

2-10 Zonal and meridional velocity components from $\mathrm{Fr}^{-1}=0.4$ simulation averaged over several inertial periods. . . . . . . . . . . . .

2-11 Vertical profiles of the total energy dissipation rate (gray) and energy conversion from the mean subinertial flow into internal waves (black) $\left(\mathrm{W} \mathrm{kg}^{-1}\right)$ for $\mathrm{Fr}^{-1}=0.4$ simulation. 
2-12 Diagram of energy pathways. Energy conversion and dissipation values $\left(\mathrm{mW} \mathrm{m}^{-2}\right)$ are from $F r^{-1}=0.4$ simulation. Values inside boxes represent growth (time derivative) of kinetic energy. . . . . . . . .

2-13 Wave zonal velocity $\left(\mathrm{m} \mathrm{s}^{-1}\right)$ from the linear solution in (2.32) corresponding to $\mathrm{Fr}^{-1}=0.4$ simulation. . . . . . . . . . . . . . 82

2-14 Vertical profiles of energy dissipation rate $\left(\mathrm{W} \mathrm{kg}^{-1}\right)$ from different simulations. . . . . . . . . . . . . . . . . . . 83

2-15 Energy dissipation rate $\left(\mathrm{mW} \mathrm{m}^{-2}\right)$ integrated in bottom $1 \mathrm{~km}$. . . . . 84

3-1 Buoyancy frequency $\left(\mathrm{s}^{-1}\right)$ in logarithmic scale from the ALBATROSS section, Drake Passage. . . . . . . . . . . . . . 107

3-2 Flow speed $\left(\mathrm{m} \mathrm{s}^{-1}\right)$ from the ALBATROSS section, Drake Passage. . 108

3-3 Buoyancy frequency $\left(\mathrm{s}^{-1}\right)$ in logarithmic scale from the P18 section, Southeast Pacific. . . . . . . . . . . . . . . . . . . . . . 109

3-4 Flow speed $\left(\mathrm{m} \mathrm{s}^{-1}\right)$ from the P18 section, Southeast Pacific. . . . . . 110

3-5 Bottom stratification $\left(\mathrm{s}^{-1}\right)$ for the Drake Passage and the Southeast Pacific regions. . . . . . . . . . . . . . . . . 111

3-6 Bottom speed $\left(\mathrm{m} \mathrm{s}^{-1}\right)$ for the Drake Passage and the Southeast Pacific

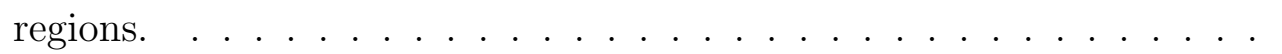

3-7 Multi-beam topography data (British Antarctic Survey) for the Drake Passage region superimposed on the bathymetry data from satellite altimetry (Smith and Sandwell, 1997). . . . . . . . . . . . 113

3-8 Two-dimensional topography spectrum estimated using multi-beam topography data from the Drake Passage (colored lines) and fitted with model spectrum (Goff and Jordan, 1988) (black lines); characteristic wavenumber range for lee wave radiation (yellow) . . . . . . . . .

3-9 Satellite bathymetry of the Southeast Pacific region (m); LADCP stations along P18 section (blue), shipboard topographic data (red). . .

3-10 One-dimensional topography spectrum estimated along ship tracks from the combination of satellite bathymetry and shipboard topography. . 116 
3-11 Effective topographic spectra for the Drake Passage region (red) and the Southeast Pacific region (blue) corresponding to the lower (dashed) and the upper (solid) limits of wave energy radiation. . . . . . . . . 117

3-12 Inverse Froude number spectrum (black) for a station in the Polar Front of the ACC in the Drake Passage region, lee wave radiation range (yellow).

3-13 Inverse Froude number estimates for the Drake Passage (red) and the Southeast Pacific (blue) regions estimated using the lower (dashed) and the upper (solid) limits of effective topography estimate. . . . . . 119

3-14 Energy radiation estimate $\left(\mathrm{mW} \mathrm{m}^{-2}\right)$ along the ALBATROSS section in the Drake Passage region. . . . . . . . . . . . . . . . . . . . . . 120

3-15 Energy radiation estimate $\left(\mathrm{mW} \mathrm{m}^{-2}\right)$ along the $\mathrm{P} 18$ section in the Southeast Pacific region. . . . . . . . . . . . . . . .

3-16 Bottom topography spectra used for simulations characteristic of the Drake Passage (red squares) and in the Southeast Pacific (blue squares) regions; corresponding Goff's model spectra are shown as black curves. 122

3-17 Snapshot of the wave zonal velocity $\left(\mathrm{m} \mathrm{s}^{-1}\right)$ from the Drake Passage simulation. . . . . . . . . . . . . . . . . . . . . 123

3-18 Snapshot of the wave zonal velocity $\left(\mathrm{m} \mathrm{s}^{-1}\right)$ from the Southeast Pacific simulation. . . . . . . . . . . . . . . . . . . . . 124

3-19 Time evolution of the zonally averaged meridional velocity component $\left(\mathrm{m} \mathrm{s}^{-1}\right)$ from the Drake Passage simulation. . . . . . . . . . . . . 125

3-20 Time evolution of the zonally averaged meridional velocity component $\left(\mathrm{m} \mathrm{s}^{-1}\right)$ from the Southeast Pacific simulation. . . . . . . . . . . . 126

3-21 Profiles of energy dissipation rate $\left(\mathrm{W} \mathrm{kg}^{-1}\right)$ diagnosed from the Drake Passage (red) and the Southeast Pacific (blue) simulations. . . . . . . 127

4-1 Bottom topography used in the 3-D simulations. Horizontal and ver-

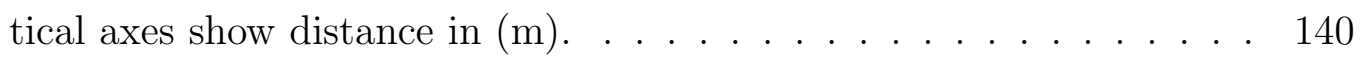


4-2 Time evolution of zonal and meridional velocity components $\left(\mathrm{m} \mathrm{s}^{-1}\right)$ averaged in both zonal and meridional directions from $h_{T}=60 \mathrm{~m}$ simulation: (upper panel) zonal velocity component, a deviation from an externally prescribed $0.1 \mathrm{~m} \mathrm{~s}^{-1}$ zonal mean flow, (lower panel) meridional velocity component. . . . . . . . . . . . . . . . . . 141

4-3 Evolution of the bottom value of inertial oscillation amplitude $\left(\mathrm{m} \mathrm{s}^{-1}\right)$ from different simulations. . . . . . . . . . . . . . . . . . . . . . . . . 142

4-4 Profiles of the vertical energy flux $\left(\mathrm{mW} \mathrm{m}^{-2}\right)$ from different simulations. 143

4-5 Energy conversion rate at the bottom as a function of $\mathrm{Fr}^{-2}$ : diagnosed from 2-D simulations (blue circles) and from 3-D simulations (red squares), predicted from linear lee wave theory (dashed lines), and predicted from linear theory for inertial harmonics (solid lines). . 144

4-6 Snapshot of an isopycnal surface (red) roughly $100 \mathrm{~m}$ above topography from the $h_{T}=60 \mathrm{~m}$ simulation and underlying bottom topography (blue). 145

4-7 Vertical profiles of energy dissipation rate $\left(\mathrm{W} \mathrm{kg}^{-1}\right)$ from different simulation. . . . . . . . . . . . . . . . . . 146

5-1 Bathymetry of the Southern Ocean (Smith and Sandwell, 1997). . . . 158

5-2 Map of the rms topographic height of the Southern Ocean computed over non-overlapping $1^{o} \times 1^{o}$ bins. . . . . . . . . . . . . . . . 158 


\section{Chapter 1}

\section{Introduction}

The Southern Ocean plays a significant role in the global climate system. It hosts the Antarctic Circumpolar Current (ACC), the largest zonal current in the world, which links the Atlantic, Indian and Pacific Oceans. The associated Meridional Overturning Circulation (MOC) is crucial to the meridional transport and storage of heat, salt, $\mathrm{CO}_{2}$ and other properties into the ocean. The MOC can be described by an upper and a lower cell (Speer et al., 2000). Theories suggest that the upper cell is driven by atmospheric forcing at the surface with an adiabatic return flow at depth (Rintoul et al., 2001; Marshall and Radko, 2003; Olbers et al., 2004). Much less is known about the dynamics of the lower cell, except that diabatic mixing is essential to explain the latitudinal change in deep water mass properties (Ito and Marshall, 2008). Inverse analyses of the Southern Ocean hydrography concur that high mixing rates in the deep Southern Ocean are required to close the heat and salt budgets (Ganachaud and Wunsch, 2000; Sloyan and Rintoul, 2001).

Several observational estimates from high wavenumber signatures in density and velocity profiles show enhanced turbulent mixing in the Southern Ocean associated with internal wave breaking (Polzin and Firing, 1997; Naveira-Garabato et al., 2004). This mixing is typically concentrated in the bottom kilometer in regions above rough topography. Enhanced abyssal mixing has been recently linked to internal tides in the Brazil Basin region (Polzin et al., 1997; Ledwell et al., 2000; St.-Laurent et al., 2001). However, in the Southern Ocean tidal currents are weak, $O(1) \mathrm{cm} \mathrm{s}^{-1}$ (Egbert 
et al., 1994), and abyssal flows are dominated by geostrophic eddies of $O(10) \mathrm{cm} \mathrm{s}^{-1}$ (Naveira-Garabato et al., 2003). It has been suggested (Polzin and Firing, 1997; Naveira-Garabato et al., 2004) that enhanced abyssal mixing in the Southern Ocean is sustained primarily by internal waves generated by the deep ocean geostrophic flows interacting with bottom topography.

The goal of this thesis is to understand what drives abyssal mixing in the Southern Ocean. The main hypothesis is that geostrophic eddies interact with small-scale topographic hills and generate internal waves which radiate into ocean interior, break and sustain observed mixing. First, this problem is studied for an idealized mean flow and topography using linear theory and numerical simulations. Then, linear theory and simulation results are used to estimate the contribution of topographic internal waves to mixing in the Southern Ocean from available data.

\subsection{Observational estimates of abyssal mixing}

Observations show that there are large variations in the levels of turbulent mixing throughout the ocean. Diapycnal diffusivities change by several orders of magnitude from low values of $10^{-5} \mathrm{~m}^{2} \mathrm{~s}^{-1}$ in the ocean thermocline (Ledwell et al., 1998), taken now as the typical background value, to greatly enhanced values of up to $10^{-2} \mathrm{~m}^{2} \mathrm{~s}^{-1}$ in the deep ocean above regions of rough topography in the Brazil Basin (Polzin et al., 1997; Ledwell et al., 2000), in the Southeast Indian Ocean (Polzin and Firing, 1997; Kunze et al., 2006), and in the Drake Passage region (Naveira-Garabato et al., 2004).

Estimates of turbulent dissipation rate $\epsilon$ and diapycnal diffusivity $\kappa_{\rho}$ in the Southern Ocean are inferred indirectly from the LADCP and CTD data (Polzin and Firing, 1997; Naveira-Garabato et al., 2004; Kunze et al., 2006). These data resolve vertical scales from full water depth down to about $50 \mathrm{~m}$. Fine-scale parametrizations (Gregg, 1989; Polzin et al., 1995) are then used to relate the $50 \mathrm{~m}$ shear to the dissipation $\epsilon$ at molecular scales. These parameterizations rely on the assumption that molecular dissipation of kinetic energy can be related to larger scale, i.e. $O(50) \mathrm{m}$, properties of the fine-scale internal wavefield. The combined error from these assumptions is 
estimated to lead to an uncertainty of a factor of 2 to 3 in the final estimates of $\epsilon$.

Polzin and Firing (1997) used velocity (LADCP), temperature, salinity and pressure (CTD) data from a South Indian section which extends from $30^{\circ} \mathrm{S}, 90^{\circ} \mathrm{E}$ on the Broken Plateau to $64^{\circ} \mathrm{S}, 82^{\circ} \mathrm{E}$ south of the Kerguelen Plateau (Fig. 1-1) to estimate turbulent energy dissipation and diapycnal diffusivity. They examined two groups of 12 stations centered respectively at $\sim 35^{\circ} \mathrm{S}$ and $\sim 55^{\circ} \mathrm{S}$, the first region being in the subtropical gyre of the Indian Ocean and the second poleward of the Antarctic Polar Front. The average vertical diffusivity from $55^{\circ} \mathrm{S}$ is estimated to be $4.4 \times 10^{-4} \mathrm{~m}^{2} \mathrm{~s}^{-1}$ which is 40 times larger than in the ocean thermocline (Gregg, 1989) and more than four times larger than the diffusivity at $35^{\circ} \mathrm{S}$. Depth integrated dissipation rates are estimated to be 4.6 and $0.95 \mathrm{~mW} \mathrm{~m}^{-2}$ for $55^{\circ} \mathrm{S}$ and $35^{\circ} \mathrm{S}$, respectively.

Polzin and Firing (1997) further show that the internal wave field is much more energetic at $55^{\circ} \mathrm{S}$ than at $35^{\circ} \mathrm{S}$. Using rotary spectra the authors determine that there is an upward radiation of internal waves, implying their generation by bottom currents impinging over rough topography. Bottom flows in the ocean are typically dominated by geostrophic eddies and barotropic tides which can generate, respectively, lee waves and internal tides. However, the barotropic tidal flows do not vary significantly between $35^{\circ} \mathrm{S}$ and $55^{\circ} \mathrm{S}$. The major difference between these two regions is the background geostrophic currents (Fig. 1-1). The rms of the depth-averaged velocity is $\sim 20 \mathrm{~cm} \mathrm{~s}^{-1}$ at $55^{\circ} \mathrm{S}$ and $\sim 4 \mathrm{~cm} \mathrm{~s}^{-1}$ at $35^{\circ} \mathrm{S}$ (Polzin and Firing, 1997).

Kunze et al. (2006), applying a similar fine-scale parameterization to a number of full depth lowered ADCP profiles collected during the World Ocean Circulation Experiment (WOCE), find somewhat lower values of $\kappa_{\rho}$ and $\epsilon$ in these regions. However, the main conclusion stands: mixing is enhanced at $55^{\circ} \mathrm{S}$ compared to $35^{\circ} \mathrm{S}$ and it is correlated with the magnitude of the bottom flow. Thus, it is plausible to speculate that the enhanced mixing at $55^{\circ} \mathrm{S}$ is supported by internal lee waves generated by the bottom current flowing over small-scale topographic features.

Naveira-Garabato et al. (2004) in an independent study also report dissipation rates from LADCP and CTD data (together with a fine-scale parameterization) in the Southeast Pacific and the Drake Passage sectors of the Southern Ocean (Fig. 1- 
2). Bottom topography is characterized by a series of abyssal plains in the Southeast Pacific and by complex structures in Drake Passage. Fig. 1-3 shows that the dissipation rates change dramatically between the two regions. In the Southeast Pacific over the abyssal plains the diffusivity is relatively low, about $10^{-5}-10^{-4} \mathrm{~m}^{2} \mathrm{~s}^{-1}$, with a corresponding vertically integrated dissipation rate of the order of $1 \mathrm{~mW} \mathrm{~m}^{-2}$. In Drake Passage, the diffusivity increases to values as large as $10^{-2} \mathrm{~m}^{2} \mathrm{~s}^{-1}$ and the dissipation rate is an an order of magnitude larger at $10 \mathrm{~mW} \mathrm{~m}^{-2}$ [The vertically integrated dissipation rate estimates quoted in (Naveira-Garabato et al., 2004) are biased high by a factor of 2 to 3 . In the letter attached at the end of the chapter, A. Naveira-Garabato and K. Polzin explain that the overestimate resulted from an oversight of not including a correction for the near-inertial frequency content of the observed velocity finestructure. The numbers with the correction are $0.8 \mathrm{~mW} \mathrm{~m}^{-2}$ in the Southeast Pacific and to $9 \mathrm{~mW} \mathrm{~m}^{-2}$ in Drake Passage.]

The correlation between high dissipation rates and bathymetric roughness is interpreted by Naveira-Garabato et al. (2004) as evidence that abyssal mixing is generated by internal waves radiated by deep flows impinging on rough topography. Although it is difficult to determine from data whether enhanced dissipation is sustained by tidal or geostrophic flows, the dominance of eddy flows in the Southern Ocean point towards a greater importance of geostrophic sources.

To summarize, turbulent energy dissipation rate and diapycnal diffusivity estimates for the Southern Ocean inferred from the observations suggest that mixing is greatly enhanced in the bottom kilometer above rough topography regions and it is accompanied by upward internal wave energy propagation. Dissipation rates increase in regions of geostrophic mean flows and rough topography. These characteristics are consistent with the hypothesis that topographic waves are generated by geostrophic flows, radiate upward and break, sustaining mixing. However, the hypothesis is not yet supported by a sound theoretical framework. 


\subsection{Major contributors to mixing}

In the global ocean abyssal flows are dominated by tides, particular the lunar $M_{2}$ tide, and by geostrophic motions. Barotropic tidal currents reach up to $O(5) \mathrm{cm} \mathrm{s}^{-1}$ in most of the ocean (Egbert et al., 1994). Typical velocities due to geostrophic eddies are of $O(1) \mathrm{cm} \mathrm{s}^{-1}$, but they reach $O(10) \mathrm{cm} \mathrm{s}^{-1}$ in western boundary currents and abyssal canyons (Dickson, 1990). Internal tides can radiate from large topographic features, like ridges, while geostrophic flows of $O(1) \mathrm{cm} \mathrm{s}^{-1}$ can radiate only from small topographic features with scales shorter than $100 \mathrm{~m}$. Hence, internal wave radiation in the global ocean is believed to be dominated by tidal flows. In the Southern Ocean, however, tidal velocities are smaller, $O(1) \mathrm{cm} \mathrm{s}^{-1}$ (Egbert et al., 1994), and an inverse cascade unarrested by lateral boundaries allows geostrophic eddies to develop a large barotropic component resulting in $O(10) \mathrm{cm} \mathrm{s}^{-1}$ abyssal flows (Polzin and Firing, 1997; Naveira-Garabato et al., 2003). It is therefore an open question whether topographic internal waves are generated primarily by geostrophic or tidal flows in the Southern Ocean.

\subsubsection{Internal tides}

Internal tides are generated in stratified regions where barotropic tidal currents flow over bottom topography, provided that their frequency $\omega$ is in the range $f<\omega<N$, where $N$ is the buoyancy frequency and $f$ is the Coriolis frequency. Thus, diurnal internal tides can only propagate equatorward of about $\pm 30^{\circ}$ latitude, while semidiurnal tides can freely propagate up to $\pm 74.5^{\circ}$. Beyond these latitudes internal tides are trapped over topography.

The expression for the vertical energy flux $E_{\text {tides }}$ radiated by a barotropic tidal current flowing over topography is reported here to illustrate some fundamental properties of internal tide generation in the ocean. The reader is referred to the vast literature on the subject, e.g. (Bell, 1975a,b; Garrett and Kunze, 2007), for a detailed discussion. The expression is based on a two-dimensional linear theory for the interaction of a barotropic tide, $U(t)=U_{0} \cos \omega t$, with a monochromatic topogra- 
phy, $h(x)=h_{0} \cos k x$, in the limit of sub-critical topographic slope and small tidal excursion,

$$
E_{\text {tides }}=\frac{1}{4} \rho_{0} \omega^{-1}\left[\left(N^{2}-\omega^{2}\right)\left(\omega^{2}-f^{2}\right)\right]^{1 / 2} U_{0}^{2} k h_{0}^{2},
$$

where $\rho_{0}$ is a reference density. The energy flux estimate $E_{\text {tides }}$ is averaged over one tidal period, $2 \pi / \omega$, and one topographic wavelength, $2 \pi / k$.

The radiated energy scales quadratically with both the barotropic tide amplitude $U_{0}$ and the topographic amplitude $h_{0}$. The frequency of internal tides $\omega$ is set by the barotropic tide, independent of the topographic scale $k$. Hence tides can radiate from a wide range of topographic scales provided that $f<\omega<N$, as long as their vertical scale is smaller that the local depth of the ocean (The constraint due to finite ocean depth is important as pointed out by Khatiwala (2003), because it suppresses otherwise very energetic modes). The topographic spectrum in the ocean abyss is dominated by large features, like ridges, with a steep roll-off at high wavenumbers (Smith and Sandwell, 1997). The dependence of the energy flux on topography implies that the internal tide energy flux is dominated by the largest topographic scales corresponding to isolated sea mountains and ridges on scales larger than 20-100 km.

Large topographic scales generate low-mode internal tides with large Richardson numbers (Garrett and Kunze, 2007). Wave-wave interactions seem to be ineffective at rapidly transferring energy from the low-mode waves, that dominate the energy flux, to higher modes (St.-Laurent and Garrett, 2002). The present understanding is that only a small fraction of energy flux, less than $30 \%$, is generated at high modes, i.e. with vertical scales short enough to interact nonlinearly and dissipate locally (St.-Laurent and Garrett, 2002). Low-mode internal tides can either interact with topography, as they bounce back from the surface, and scatter their energy to higher modes dissipating locally (Polzin, 2008), or radiate away horizontally and possibly contribute to mixing at remote locations (St.-Laurent and Garrett, 2002).

Internal wave generation by the dominant $\mathrm{M}_{2}$ tide component is estimated globally by Nycander (2005) using the linear theory of internal wave generation developed by Bell (1975a,b) and modified by Llewellyn-Smith and Young (2002) to account for 
the finite ocean depth. Nycander (2005) uses tidal velocities from the global tidal model of Egbert and Erofeeva (2002) and bottom topography from satellite altimetry (Smith and Sandwell, 1997). In the Drake Passage region, the tidal energy flux radiated into the ocean interior from topography deeper than $2 \mathrm{~km}$ is estimated to be about 1-2 $\mathrm{mW} \mathrm{m}^{-2}$. Thus, it appears that tidal energy available to support local mixing is an order of magnitude smaller than the dissipation rates estimated from LADCP data by Naveira-Garabato et al. (2004). Although radiation of internal tides and their contribution to mixing might be globally important, this process does not seem to explain the enhanced mixing observed in the Southern Ocean.

The linear theory estimates of tidal radiation presented so far assume that topographic slopes are sub-critical, i.e. the tidal wave slope is steeper than the topographic slope. At smaller scales topography is characterized by relatively steep slopes and linear theory is formally invalid. Theories and numerical simulations (St.-Laurent and Garrett, 2002; Balmforth et al., 2002; Llewellyn-Smith and Young, 2003; Legg and Huijts, 2006) suggest that, at critical and super-critical slopes, energy radiation and, more importantly, the fraction of local dissipation can significantly increase above the linear theory prediction. This suggests that the estimate of tidal dissipation of Nycander (2005) might be biased low. But the fundamental result holds. The bulk of tidal energy is radiated in low-modes from large topographic scales which are well described by linear theory and contribute little to dissipation.

\subsubsection{Lee waves}

The generation of mountain lee waves propagating upward and depositing their momentum at higher altitudes is an important process for the atmospheric circulation. In the ocean, lee wave generation is generally considered of little importance because bottom geostrophic flows are traditionally believed to be weak of $O(1) \mathrm{cm} \mathrm{s}^{-1}$ (Dickson, 1990). However, in the Southern Ocean, where bottom geostrophic flows are as large as $O(10) \mathrm{cm} \mathrm{s}^{-1}$ (Polzin and Firing, 1997; Naveira-Garabato et al., 2003), lee wave generation can be an important source of internal waves.

The theory of lee wave generation is reviewed here to better illustrate the proper- 
ties of lee waves. For simplicity the discussion focuses on a two-dimensional, horizontally periodic, and vertically semi-infinite domain with idealized bottom topography given by $h(x)=h_{0} \cos k x$. Assuming small amplitude waves and considering a constant, vertically uniform mean flow $U_{0}$ and stratification $N$, the linearized equations governing lee wave dynamics can be written as,

$$
\begin{aligned}
U_{0} u_{x}-f v & =-p_{x}, \\
U_{0} v_{x}+f u & =0, \\
U_{0} w_{x} & =-p_{z}+b, \\
U_{0} b_{x}+N^{2} w & =0 \\
u_{x}+w_{z} & =0 .
\end{aligned}
$$

where $\mathbf{u}=(u, v, w), b$, and $p$ are the wave velocity, buoyancy, and pressure fields respectively, and $f$ is the Coriolis parameter. This set of equations can be reduced to a single equation for the vertical velocity $w$,

$$
U_{0}^{2}\left(w_{x x}+w_{z z}\right)_{x x}+N^{2} w_{x x}+f^{2} w_{z z}=0 .
$$

Substituting plane wave solution of the form $w \sim e^{i(k x+\mu z)}$, one obtains the vertical scale of the wave, $\mu$, in terms of the other parameters of the problem,

$$
\mu^{2}=k^{2} \frac{N^{2}-U_{0}^{2} k^{2}}{U_{0}^{2} k^{2}-f^{2}}
$$

Lee waves can radiated upward when their frequency $U_{0} k$ is in the range $f<U_{0} k<N$ so that $\mu$ is real. Outside this range $\mu$ is imaginary and the waves are bottom-trapped. The vertical scale of radiated waves is $\mu \approx N / U_{0}$, except for waves whose frequencies are close to $f$ or $N$.

The radiation problem is fully determined by imposing two boundary conditions. First, it is required that energy is radiated upward. Second, the bottom velocity must satisfy a no-normal flow condition at the topography. In order to make analytical 
progress this bottom boundary condition is linearized,

$$
\left.w\right|_{z=0}=U_{0} h_{x} .
$$

This approximation is valid if the topography is sub-critical, i.e. if the ratio of topographic slope $k h_{0}$ to the lee wave slope $k \mu^{-1}$ is small,

$$
F r^{-1}=\frac{k h_{0}}{k \mu^{-1}}=h_{0} \mu \approx \frac{N h_{0}}{U_{0}} \ll 1 .
$$

The inverse Froude number, $\mathrm{Fr}^{-1}$, is the fundamental nondimensional parameter that characterizes the properties of radiated lee waves. There are three different dynamical regimes. In the sub-critical regime, $F r^{-1} \ll 1$, lee wave generation is well described by linear theory. Lee waves radiate from topography and transport momentum and energy upward. In the critical regime, $\mathrm{Fr}^{-1} \sim 1$, nonlinear effects become important and waves start breaking above topography. Low-level dynamics are characterized by hydraulic jumps and turbulence in the lee of the mountain. At $\mathrm{Fr}^{-1} \gg 1$, the super-critical regime, stratification suppresses vertical motions and mean flow - topography interaction is characterized by upstream blocking of the flow and downstream formation of counter-rotating vortices.

The expression for the lee wave vertical energy flux, $E_{l w}$, is given by,

$$
E_{l w}=\frac{1}{2} \rho_{0}\left[\left(N^{2}-U_{0}^{2} k^{2}\right)\left(U_{0}^{2} k^{2}-f^{2}\right)\right]^{1 / 2} U_{0} h_{0}^{2},
$$

The major difference from the corresponding expression for internal tides in (1.1) is that the frequency of lee waves, $U_{0} k$, is set by the topographic scale, $k$. It is still true that radiation is dominated by larger scales, in the admissible range, because of the red nature of the topographic spectrum. But the admissible range $\left(f / U_{0}, N / U_{0}\right)$ is limited to short scales of less than $O(1) \mathrm{km}$, even for the strongest abyssal flows of $O(10) \mathrm{cm} \mathrm{s}^{-1}$.

Despite the fact that radiation is limited to small abyssal hills, geostrophic flows are more efficient at generating nonlinear waves and breaking. That is because the 
longer the lee wave, the shorter is its vertical scale (unlike for tides). This results in small Richardson numbers and favors local wave breaking.

A lot is known about the radiation of lee waves in the atmosphere (Wurtele et al., 1996). However the problem needs to be revisited in the oceanographic context, because of the different mean flow and stratification in the two fluids. Typical values of velocity and stratification in the atmosphere are $U_{0} \approx 10 \mathrm{~m} \mathrm{~s}^{-1}$ and $N \approx 10^{-2} \mathrm{~s}^{-1}$, respectively. These values imply that lee waves can radiate from topographic scales in the range from 6 to $600 \mathrm{~km}$ where radiation is dominated by isolated features at the largest scales, i.e. by mountain ranges. The associated vertical scale of lee waves is of the order of $6 \mathrm{~km}$ which is somewhat shorter than the tropopause height of 10-15 $\mathrm{km}$.

Wave generation in the atmosphere is typically characterized by one of two regimes. First, at small $\mathrm{Fr}^{-1}$ (mostly associated with small topographic features), the radiated waves are linear and can propagate up to middle atmospheric levels (30-100 $\mathrm{km})$. At these altitudes, changes in the background conditions, such as wind speed, stratification and density, lead to accumulation of internal wave energy and strong nonlinear steepening, causing wave overturning and breaking. The resulting deposition of momentum is believed to drive the mean atmospheric circulation at those levels and is parameterized in numerical models based on the saturation hypothesis of Lindzen (1981). Second, at larger $F r^{-1}$, typically associated with high topographic features, the generated waves are highly nonlinear and break right above topography. Although these phenomena can be very violent and destructive, they are trapped close to topography and are unimportant for the dynamics of the upper atmosphere.

Lee wave radiation in the ocean and is typically somewhere between the two regimes dominating in the atmosphere. For abyssal flow speeds of $U \approx 10 \mathrm{~cm} \mathrm{~s}^{-1}$ and stratification of $N \approx 10^{-3} \mathrm{~s}^{-1}$, typical of the Southern Ocean, wave generation occurs for topographic scales in the range from $600 \mathrm{~m}$ to $6 \mathrm{~km}$. At these scales, the ocean bottom topography is characterized by abyssal hills, which cover approximately $80 \%$ of the world's seafloor, rather than by isolated mountains and ridges. As a result, the radiated waves are multichromatic and the energy flux is dominated by vertical 
scales of a few hundred meters, significantly smaller than the dominant scale of oceanic internal tides or atmospheric lee waves, and much smaller than the full ocean depth.

Inverse Froude numbers in the ocean are not very large and span the range between the sub-critical and critical regimes. The combination of a multichromatic wave field, small vertical scales and intermediate Froude numbers implies that wave-wave interactions are important for dissipation of lee waves in the ocean. The situation is quite different from the atmospheric one, where radiation is mostly in the form of monochromatic waves which break individually as a result of high $\mathrm{Fr}^{-1}$ or by encountering critical levels. There are no critical levels in the ocean for typical velocity and stratification profiles. As waves propagate upward into the ocean interior they usually encounter larger stratification and background flow velocity such that the Richardson number stays above the critical value. A major question addressed in

this thesis is whether lee waves generated in the Southern Ocean can break and sustain the observed dissipation.

\subsection{Large-scale flow - topography interaction}

This section briefly explains the dynamics of the large-scale flow - topography interaction described in the thesis. Results of this thesis show that, at the finite inverse Froude numbers characteristic of the Southern Ocean, the interaction of a large-scale geostrophic flow with a small-scale topography differs from the classical lee wave problem. The internal waves feedback on the zonally averaged flow and drive spontaneous growth and subsequent equilibration of inertial oscillations in the bottom several hundred meters. In the statistical steady state, the zonally averaged flow becomes a superposition of a mean geostrophic flow and bottom intensified, vertically sheared inertial oscillations. This flow interacting with small-scale topography radiates time-dependent and multichromatic internal waves. Vertical shear associated with inertial oscillations triggers wave breaking and dissipation in the bottom several hundred meters. 


\subsubsection{Steady flow}

In the classical lee wave problem (Long, 1953; Bretherton, 1969), a constant flow in a stratified fluid over a variable bottom topography generates steady, upward radiating internal waves. The problem is idealized again as a uniform mean flow $U_{0}$ in a fluid with constant stratification $N$ flowing over a monochromatic bottom topography $h(x)=h_{0} \cos k x$. The solution for the wave vertical velocity $w^{\prime}$ satisfying a nonormal flow lower boundary condition and an upward energy radiation condition can be written as,

$$
w^{\prime}=-h_{0} U_{0} k \sin (k x+\mu z),
$$

where the wave horizontal wavenumber $k$ is set by the topography and the vertical wavenumber $\mu$ is given by the internal wave dispersion relation. Radiated internal waves are steady and monochromatic in both the horizontal and vertical directions. The wave amplitude depends on the topographic height $h_{0}$ and the intrinsic wave frequency $U_{0} k$ (i.e. frequency in the moving reference frame). The wave frequency in the fixed reference frame is zero.

An important property of radiating internal waves is that they are able to transport momentum and energy upward into the ocean interior. In the absence of wave dissipation, lee wave radiation in the steady state is characterized by a constant, vertically uniform wave momentum flux,

$$
\overline{u^{\prime} w^{\prime}}=-\frac{1}{2} U_{0}^{2} h_{0}^{2} k \mu<0
$$

and, therefore, a zero momentum flux divergence. A divergence appears only if the waves break and deposit their negative momentum. The resulting momentum divergence acts to slow down the zonally-averaged flow $\bar{u}$ and reduce the mean KE,

$$
\bar{u}_{t}=-\partial_{z} \overline{u^{\prime} w^{\prime}}<0 \quad \text { and } \quad\left(\frac{1}{2} \bar{u} \cdot \bar{u}\right)_{t}=-\bar{u} \cdot \partial_{z} \overline{u^{\prime} w^{\prime}}<0
$$

In a rotating system, the response of the zonally averaged flow is somewhat more complicated. The wave feedback is not confined to the zonal direction anymore, 
because the zonal and meridional equations are coupled by the Coriolis term,

$$
\begin{aligned}
& \bar{u}_{t}-f \bar{v}=-\partial_{z} \overline{u^{\prime} w^{\prime}}, \\
& \bar{v}_{t}+f \bar{u}=-\bar{p}_{y}-\partial_{z} \overline{v^{\prime} w^{\prime}}
\end{aligned}
$$

and the corresponding mean flow kinetic energy equation becomes,

$$
\left(\frac{1}{2} \overline{\mathbf{u}} \cdot \overline{\mathbf{u}}\right)_{t}=-\overline{\mathbf{u}} \cdot \partial_{z} \overline{\mathbf{u}^{\prime} w^{\prime}}-\bar{v} \bar{p}_{y}
$$

where the first term on the right hand side is the work done by the waves against the mean flow, and the second term is the work done by meridional pressure gradient on the meridional component of the mean flow.

In the absence of wave dissipation $\partial_{z} \overline{\mathbf{u}^{\prime} w^{\prime}}=0$ and the mean flow is described by a zonal, geostrophically balanced velocity component $\bar{u}$, satisfying $f \bar{u}=-\bar{p}_{y}$, and a zero meridional velocity component, $\bar{v}=0$. Both terms on the right hand side of the kinetic energy equation (1.17) are zero and the kinetic energy is in equilibrium. If there is wave dissipation in the water column, then the deposition of wave momentum acts to reduce the kinetic energy of the mean flow as in the non-rotating system. However, in the rotating system, the mean kinetic energy extracted by the waves is resupplied from the available potential energy associated with the tilt of the pressure surfaces. The large-scale meridional pressure gradient, $\bar{p}_{y}$, acting on the mean meridional flow, $\bar{v}$, represents the energy conversion from available potential energy into mean kinetic energy ${ }^{1}$. Thus, in a rotating system, internal wave damping acts to slow down the mean flow by extracting both its kinetic and potential energy.

In addition to a subinertial response in the zonally averaged flow, deposition of

\footnotetext{
${ }^{1}$ Work done by the meridional pressure gradient on the meridional component of the mean flow, $\bar{v} \bar{p}_{y}$, can be expressed in terms of the energy conversion from potential energy into kinetic energy, $\bar{w} \bar{b}$,$$
\bar{v} \bar{p}_{y}=(\bar{v} \bar{p})_{y}-\bar{p} \bar{v}_{y}=(\bar{v} \bar{p})_{y}+\bar{p} \bar{w}_{z}=(\bar{v} \bar{p})_{y}+(\bar{w} \bar{p})_{z}-\bar{w} \bar{p}_{z}=(\bar{v} \bar{p})_{y}+(\bar{w} \bar{p})_{z}-\bar{w} \bar{b}
$$

where the hydrostatic relationship $\bar{p}_{z}=b$ has been used. Hence $\bar{v} \bar{p}_{y}$ is the local conversion from available potential energy $\bar{w} \bar{b}$, plus a non-local flux term $(\bar{v} \bar{p})_{y}+(\bar{w} \bar{p})_{z}$ representing work done by remote pressure forces.
} 
internal wave momentum in a rotating system also drives motions on shorter time scales. Analogous to a wind stress at the surface of the ocean, internal wave momentum stresses can force near-inertial oscillations and higher frequency internal waves.

\subsubsection{Oscillatory flow}

A major difference between steady and time-dependent flows over bottom topography is that the time-dependent flow generates a wave field with time-dependent momentum fluxes which results in time-dependent forcing acting on the zonally averaged flow. The interaction of the time-dependent flow oscillating at frequency $\omega_{0}$ with bottom topography has been shown to radiate internal waves not only at the fundamental frequency $\omega_{0}$, but also at all of its harmonics (Bell, 1975). For a simple oscillatory flow, $U(t)=U_{0} \cos \omega_{0} t$, over a monochromatic topography, the linear solution for the wave vertical velocity $w^{\prime}$ can be written as,

$$
w^{\prime}=-\sum_{n=-\infty}^{\infty} h_{0} \omega_{n} J_{n}\left(\frac{U_{0} k}{\omega_{0}}\right) \sin \left(k x-U_{0} k / \omega_{0} \sin \omega_{0} t+\mu_{n} z+\omega_{n} t\right)
$$

where $\omega_{n}=n \omega_{0}$ are the harmonics of the fundamental frequency $\omega_{0}, \mu_{n}$ are the harmonic vertical wavenumbers, and $J_{n}$ are Bessel functions of the first kind. In addition to the topographic height $h_{0}$ and frequency $\omega_{n}$, the amplitude of each harmonic is determined by the excursion parameter, $U_{0} k / \omega_{0}$, which is the ratio of the distance that a water parcel travels during one period of oscillation to the horizontal scale of the topography. If the excursion parameter is small, then a water parcel stays on one topographic bump and internal waves are radiated mostly at the fundamental frequency $\omega_{0}$. However, as the excursion parameter becomes finite, a water parcel travels over several bumps and higher harmonics of the fundamental frequency are radiated. This makes the wave field multichromatic.

The momentum flux associated with the radiation of a multichromatic field characterized by frequency $n \omega_{0}$ has steady and time-dependent components,

$$
\overline{u^{\prime} w^{\prime}}=\left.\overline{u^{\prime} w^{\prime}}\right|_{\omega=0}+\left.\overline{u^{\prime} w^{\prime}}\right|_{\omega= \pm \omega_{0}}+\left.\overline{u^{\prime} w^{\prime}}\right|_{\omega= \pm 2 \omega_{0}}+\ldots
$$


where terms on the right hand side are the flux components oscillating at harmonics of the fundamental frequency $\omega_{0}$. When time-dependent waves break and deposit their momentum, they result in a time-dependent forcing on the zonally averaged flow through the divergence of the momentum flux according to (1.15) and (1.16).

\subsubsection{Abyssal flows in the ocean}

Abyssal flows in the Southern Ocean are dominated by geostrophic flows, inertial oscillations, and tides (Nowlin et al., 1986). All these motions can radiate internal waves through interaction with bottom topography. While geostrophic flows can be regarded as quasi-steady on the internal wave time scale, inertial oscillations and tides are oscillatory flows. Combining the results for wave radiation by steady and oscillatory flows discussed above, the total internal wave momentum flux can be written as a superposition of components oscillating at zero frequency (steady), inertial frequency, $f$, tidal frequency, $\omega_{T}$, and their higher harmonics and linear combinations,

$$
\overline{u^{\prime} w^{\prime}}=\left.\overline{u^{\prime} w^{\prime}}\right|_{\omega=0}+\left.\overline{u^{\prime} w^{\prime}}\right|_{\omega= \pm f}+\left.\overline{u^{\prime} w^{\prime}}\right|_{\omega= \pm \omega_{T}}+\ldots
$$

The steady component of the momentum flux has contributions from all abyssal motions and describes the time mean internal wave radiation: this flux component drives the subinertial response of the zonally-averaged flow. The time-dependent momentum flux components force a fast-time response in the zonally averaged flow at their corresponding frequencies. The components at frequency other than $f$ have no effect on the evolution of the mean flow at subinertial time scales. However, the momentum flux component oscillating at frequency $\pm f$ is different, because $f$ is the natural oscillation frequency in the rotating system, and a forcing at $f$ drives a resonant response. As a result of this resonance, inertial oscillation grow in the bottom several hundred meters and reach a magnitude comparable to the mean flow within a few days. The inertial oscillations have large shears and significantly modify subsequent wave radiation and breaking. 


\subsection{Overview of the thesis}

The thesis is organized as follows. Chapter 2 presents two-dimensional (2-D) numerical simulations and linear theory for the generation of internal waves by a geostrophic flow impinging on a sinusoidal topography. Numerical simulations show that, at finite inverse Froude numbers, inertial oscillations develop in the bottom several hundred meters. Existing linear theory for lee wave generation is extended to the generation of internal waves by a combination of subinertial and inertial motions. Using linear theory, the inertial oscillations are shown to arise through a feedback between the geostrophic flow and the radiated internal waves. Results of linear theory and numerical simulations are compared and estimates of wave radiation and dissipation are discussed. Chapter 3 presents estimates of internal wave radiation by geostrophic flows in two regions of the Southern Ocean: Drake Passage and the Southeast Pacific. The estimates are based on the theory described in the previous chapter and available velocity, stratification and topography data. Numerical simulations with multichromatic topography characteristic of the two regions are also presented to support the estimates. Chapters 2 and 3 are written as papers and meant to be read independently. In Chapter 4, the 2-D theory is extended to a three-dimensional (3-D) mean flow over 2-D topography. The results are compared to numerical simulations and to the corresponding 2-D problem. Chapter 5 concludes and discusses the implications of the results presented in the thesis for our understanding of the dynamics of the Southern Ocean. 

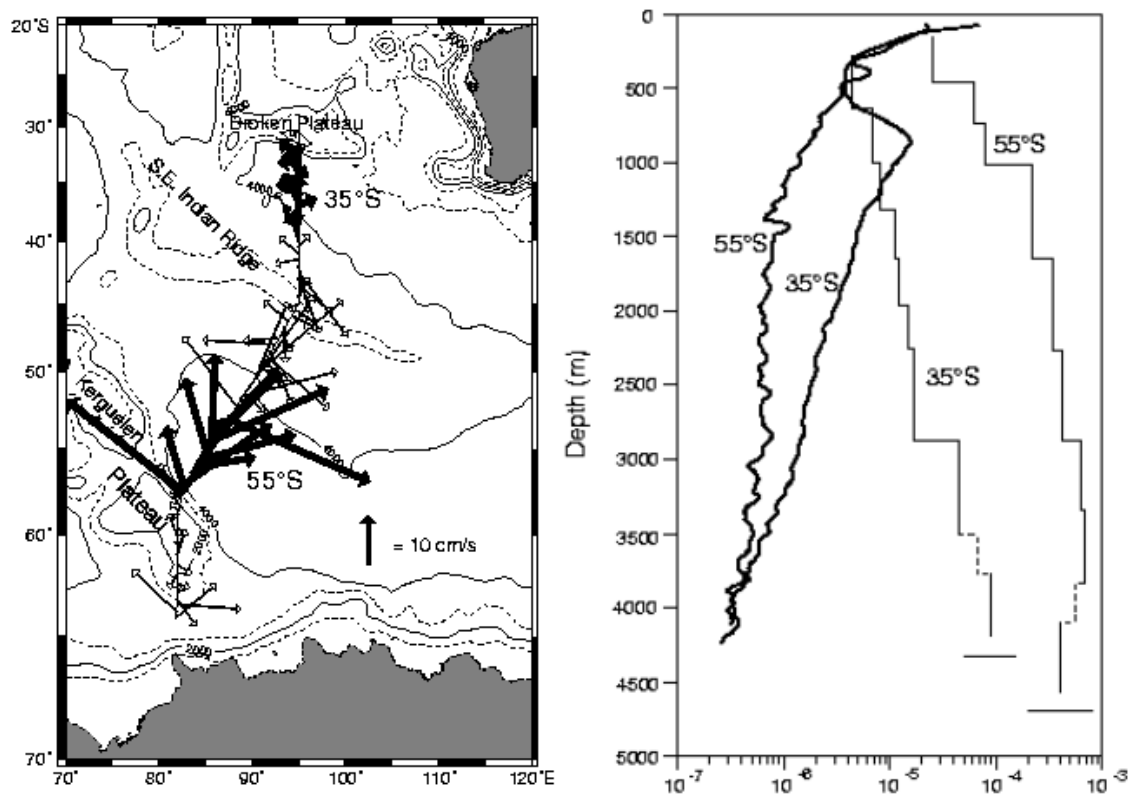

Figure 1-1: Bathymetry and depth-averaged current vectors along the I8S section (left panel). Vertical profile of $\mathrm{N}^{2}\left(\mathrm{~s}^{-2}\right)$ (thick lines) and $\kappa_{\rho}\left(\mathrm{m}^{2} \mathrm{~s}^{-1}\right)$ (thin lines) for two groups of twelve stations, one at $35^{\circ} \mathrm{S}$ and the other at $55^{\circ} \mathrm{S}$. From Polzin and Firing (1997). 


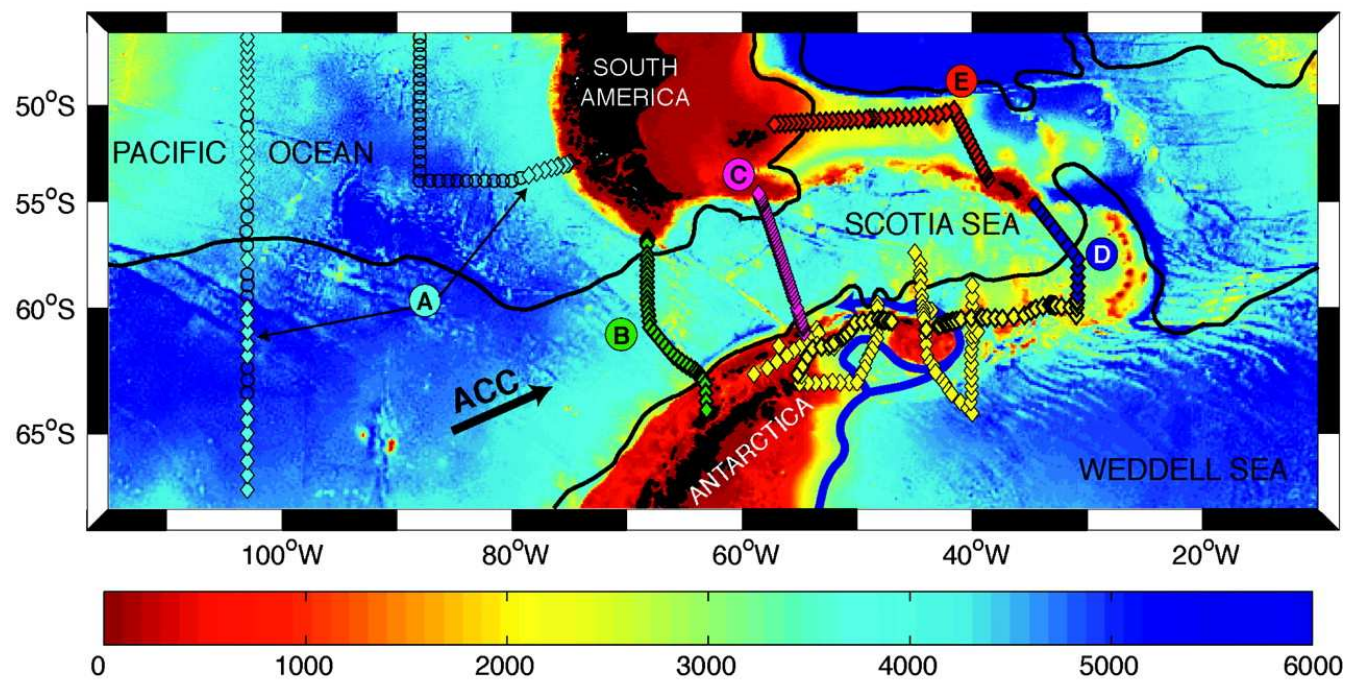

Figure 1-2: Bathymetry of the Southern Ocean region around Drake Passage. CTD and LADCP stations are shown by colored diamonds. Open circles mark stations where only CTD data are available. The black lines indicate the northern and southern boundaries of the ACC. From Naveira-Garabato et al. (2004). Reprinted with permission from AAAS.



Figure 1-3: Averaged profiles of $\mathrm{N}^{2}\left(\mathrm{~s}^{-2}\right), \epsilon\left(\mathrm{W} \mathrm{kg}^{-1}\right), \kappa_{\rho}\left(\mathrm{m}^{2} \mathrm{~s}^{-1}\right)$ for the Southeast Pacific (section A in Fig. 1-2) and the Drake Passage (section B in Fig. 1-2) regions. From Naveira-Garabato et al. (2004). Reprinted with permission from AAAS. 
Dr. Alberto C. Naveira Garabato

National Oceanography Centre

Southampton, SOl4 3ZH, U.K.

Tel.: +442380 592680

E-mail: acng@noc.soton.ac.uk

Dr. Kurt Polzin

Woods Hole Oceanngraphic Institution

Woods Holc, MA 02543, U.S.A.

Tel: $\div 15082893368$

E-mail: kpolzin@whoi.edu

$4^{\text {th }}$ September 2008

Mr. Maxim Nikurashin

Dept. of Earth, Atmospheric, and Planetary Sciences

Massachusctts Institute of Technology

Cambridge, MA 02139-4307, U.S.A.

Dear Max,

It has come to our attention that the estimates of depth-integrated dissipation rate presented in the caption of Fig. 3 of Navcita Garabato, A. C., K. L. Polzin, B. A. King, K. J. Heywood and M. Visbeck, 2004: Widespread intensc mixing in the deep Sonthern Occan, Science, 303, 210-213, arc a factor of $2-3$ high. This results from an oversight of not including a correction for the near-inertial frequency content of the observed velocity finestructure. The results appeating in the figure itsclf have the correction applied.

The numbers quoted in the caption werc: (A) $2 \times 10^{-3} \mathrm{~W} \mathrm{~m}^{-2}$, (B) $2.0 \times 10^{-2} \mathrm{~W} \mathrm{~m}^{-2}$, (C) $1.4 \times 10^{-2}$ $\mathrm{W} \mathrm{\textrm {m } ^ { - 2 }}:$ (D) $1.0 \times 10^{-2} \mathrm{~W} \mathrm{~m}^{-2}$, and (E) $5 \times 10^{-3} \mathrm{~W} \mathrm{~m}^{-2}$. With the correction, the depth integrated dissipalion estimates are (A) $8 \times 10^{-1} \mathrm{~W} \mathrm{~m}^{-2}$ :(B) $9 \times 10^{-3} \mathrm{Wm}^{-2}$, (C) $6 \times 10^{-3} \mathrm{~W} \mathrm{~m} \mathrm{~m}^{-2}$, (D) $4 \times 10^{\circ}$ ${ }^{3} \mathrm{~W} \mathrm{~m}^{-2}$, and (E) $2 \times 10^{-3} \mathrm{~W} \mathrm{~m}^{-2}$.

We wish you well in your studies of subinertial flow over bottom topography and congratulate you on your recent defence.

Yours sincerely,

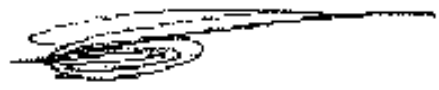

Alberto C. Naveira Garabato

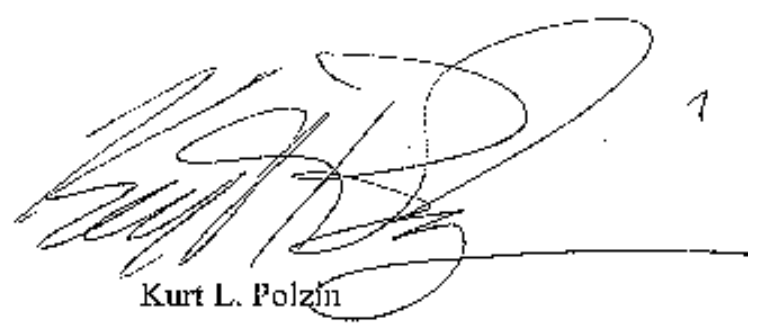




\section{Chapter 2}

\section{2-D Theory and Simulations}

\section{$2.1 \quad$ Introduction}

Turbulent mixing plays an important role in the circulation of the Southern Ocean. Observations of velocity and density fluctuations show that mixing is strongly enhanced up to a kilometer above rough bottom topography (Naveira-Garabato et al., 2004). Inverse calculations (Ganachaud and Wunsch, 2000; Sloyan and Rintoul, 2001) find that this vigorous turbulent mixing contributes crucially to the downward buoyancy flux that maintains the abyssal ocean stratification and to the upward transport of waters that closes the ocean's meridional overturning circulation. It is an open question as to what physics drives the enhanced mixing and whether these processes can be parameterized in numerical models.

Polzin et al. (1995) show that turbulent mixing in the ocean interior, away from the surface and bottom boundary layers, is typically associated with breaking internal waves. In particular they show that the intensity of turbulent fluctuations is well correlated with the local internal wave activity. Gregg (1989) uses the correlation to parameterize the levels of turbulent mixing in terms of the background oceanic internal wave spectrum described by the Garrett-Munk (GM) empirical formula (Munk, 1981). Parameterizations based on the background internal wave spectrum have remarkable skill in predicting the background turbulent mixing found in most of the ocean, but they fail to characterize regions of enhanced mixing. The diapycnal mix- 
ing inferred by Naveira-Garabato et al. (2004) in the bottom 1-2 km above rough topography in the Southern Ocean exceeds background values by 1 to 3 orders of magnitude. The vertically integrated dissipation rate averaged for a section across Drake Passage is of the order of $10 \mathrm{~mW} \mathrm{~m}^{-2}$ corresponding to a bottom diapycnal diffusivity of $10^{-2} \mathrm{~m}^{2} \mathrm{~s}^{-1}$, as opposed to background values of $10^{-2} \mathrm{~mW} \mathrm{~m}^{-2}$ and $10^{-5}$ $\mathrm{m}^{2} \mathrm{~s}^{-1}$ found in the ocean thermocline. Note that the relationship between internal wave shear and mixing (Gregg, 1989) holds. The enhancement is associated with an increased internal wave activity over GM background value.

The enhancement of turbulent mixing above rough bottom topography has been linked to the generation of internal waves by flows impinging on topography (Polzin et al., 1997; Kunze et al., 2006). The generation of this wave activity would add to the background wave field and explain the enhancement of internal wave energy and associated mixing. It is less clear what motions drive the bulk of the internal wave radiation. Nowlin et al. (1986) show, based on moored observations in Drake Passage, that the kinetic energy in the abyss is dominated by geostrophic flows, inertial oscillations, and tides. Any one of these motions can generate internal waves through interaction with bottom topography. However, most of the recent theoretical work has focused on barotropic tides, because they are believed to dominate wave radiation in the abyss (Garrett and St.-Laurent, 2002). Nycander (2005) computed the wave generation by the dominant $\mathrm{M}_{2}$ tide component using the linear theory of internal wave generation developed by Bell $(1975 \mathrm{a}, \mathrm{b})$. He estimated that the energy flux radiated by internal waves from topography deeper than $2 \mathrm{~km}$ in the Drake Passage region is at most $1-2 \mathrm{~mW} \mathrm{~m}^{-2}$. Tidal flows have been shown to radiate mostly at low vertical modes and only a small fraction, less than 30\%, goes into high modes that can dissipate locally (St.-Laurent and Garrett, 2002). Low-modes can either be influenced by topography and scatter their energy to higher wavenumbers that dissipate locally (Polzin, 2008) or radiate away and contribute to mixing in remote locations (St.-Laurent and Garrett, 2002). Hence tidal flows can account for no more than $10-20 \%$ of the observed dissipation rates. Numerical modelling of semidiurnal internal tide generation along the South Scotia Ridge (Padman et al., 
2006) also confirmes that baroclinic tidal energy is not an important contributor to mixing.

Bottom geostrophic flows are much more intense in the Southern Ocean than in most other ocean basins as a result of the nonlinear barotropization of the geostrophic eddy field. Naveira-Garabato et al. (2004) have suggested that generation of quasisteady lee waves by geostrophic flows is an alternative explanation for the enhanced wave activity in the Drake Passage region. This route is explored here with emphasis on the amount of diabatic mixing that can be supported by internal wave radiation.

In this chapter we show, using both numerical simulations and linear theory, that there are two different regimes of internal wave radiation by geostrophic flows over topography. The transition from one radiation regime to another is described by the inverse Froude number, characterizing nonlinearity of the mean flow - topography interaction. At low inverse Froude numbers, a large-scale mean geostrophic flow generates quasi-steady lee waves. In this regime, lee waves are well described by linear theory. Waves transport energy and momentum upward into the ocean interior until they break and dissipate either at a critical layer or through nonlinear wave-wave interaction. At high inverse Froude numbers, inertial oscillations develop as a result of transience in the geostrophic flow or by the adjustment of the mean geostrophic flow to the underlying topography. These oscillations are amplified through resonant feedback between the large-scale flow and internal waves. As a result, the large-scale flow becomes a superposition of a mean geostrophic flow and an inertial oscillation which produces time-dependent and multichromatic internal wave field. The vertical shear associated with inertial oscillations modulates the internal waves leading to local breaking and dissipation.

This chapter is organized as follows. In section 2, we describe the setup of the numerical experiment used to study internal wave radiation and dissipation. Simulations corresponding to the two wave radiation regimes are also discussed qualitatively. In section 3, we introduce the nondimensional parameters that characterize the properties of internal wave generation in the ocean. In section 4, we review the existing linear theory for the internal wave generation and extend it to the generation of in- 
ternal waves by subinertial and inertial motions. A major focus is the analysis of the nonlinear feedback that drives inertial oscillations and substantially modifies the radiation problem. In section 5, the numerical simulations are analyzed and compared to linear theory predictions. Conclusions are offered in section 6 .

\subsection{Simulations}

The goal of this study is to describe the generation of internal waves by geostrophic flows over topography in the abyssal ocean. The problem configuration is very idealized to focus on the essential physics. We limit the analysis to 2-D flows, but in Chapter 4 we show that our results apply to 3-D as well. The topography is represented as a series of periodic sinusoidal bumps which mimic the small-scale abyssal hills characteristic of the seafloor in regions with rough topography. A mean geostrophic flow is maintained with a depth-independent meridional pressure gradient: mesoscale eddies have weak vertical shears below the thermocline base in the Southern Ocean (Naveira-Garabato et al., 2003).

Consider first a geostrophic flow and topography parameters characteristic of the Drake Passage region. We chose Drake Passage because it's characterized by high abyssal mixing rates compared to other regions of the Southern Ocean, and because velocity, stratification, and high-resolution topography data are available in this region. LADCP and CTD data (Naveira-Garabato et al., 2002, 2003) show that in the core of the Antarctic Circumpolar Current, geostrophic eddy velocities at the ocean bottom are typically $U_{G} \sim 0.1 \mathrm{~m} \mathrm{~s}^{-1}$, the stratification is close to $N \sim 10^{-3} \mathrm{~s}^{-1}$ and the Coriolis frequency is $f \approx 10^{-4} \mathrm{~s}^{-1}$. Linear wave theory (Bell, 1975a,b) suggests that for these parameters, the lee wave energy flux is largest for topographic wavenumbers close to $k_{T} \approx 2 \pi / 2 \mathrm{~km}^{-1}$. Multibeam data for Drake Passage show that the height of topographic hills at these scales is close to $h_{T}=60 \mathrm{~m}$. The inverse Froude number, $\mathrm{Fr}^{-1}=N h_{T} / U_{G}$, characterizing nonlinearity of mean flow-topography interaction, is 0.6, i.e. wave radiation is close to critical. These parameters are given here to orient the reader of what is a regime characteristic of the Southern Ocean. In this 
chapter we vary $\mathrm{Fr}^{-1}$ and parameters related to rotation and the horizontal scale of topography to explore the other limits of the parameter space.

\subsubsection{Experiment setup}

We use the MIT general circulation model (MITgcm) (Marshall et al., 1997). The MITgcm solves the nonhydrostatic, nonlinear primitive equations using a finite-volume formulation. By running the model in the nonhydrostatic limit, hydraulic jumps and Kelvin-Helmholtz instabilities, which develop in our problem, are explicitly resolved without need of parameterizations. Representation of bottom topography by partial cells (Adcroft et al., 1997) is essential for accurate simulation of topographic internal wave generation. The MITgcm has been used for studies of wave radiation and breaking, e.g. Khatiwala (2003); Legg and Huijts (2006). In this study, we validate the model by testing the dependence of the results on grid resolution and by comparing simulations against linear theory predictions. In addition, we compare the amount of breaking and dissipation diagnosed from the model in the nonlinear regime against observations and show that they are consistent.

The domain used in the simulations is $2-\mathrm{D}$, horizontally periodic with a uniform resolution of $\Delta x=12.5 \mathrm{~m}$ in the horizontal, and of variable resolution in the vertical. The vertical grid spacing is set to $\Delta z=5 \mathrm{~m}$ in the bottom $2 \mathrm{~km}$ and it is gradually stretched to $\Delta z=300 \mathrm{~m}$ in the region above. The domain size is $L_{x} \times H_{z}=2 \mathrm{~km}$ $\times 7 \mathrm{~km}$. A sponge layer is applied between $2 \mathrm{~km}$ above the topography and the the top boundary where buoyancy and momentum are damped with a timescale of 4 hours. The sponge layer absorbs upward propagating internal waves. A uniform stratification of $N=10^{-3} \mathrm{~s}^{-1}$ and a Coriolis parameter of $f=10^{-4} \mathrm{~s}^{-1}$ are used. Horizontal and vertical viscosity and diffusivity are set to $10^{-2} \mathrm{~m}^{2} \mathrm{~s}^{-1}$ and $10^{-3} \mathrm{~m}^{2} \mathrm{~s}^{-1}$ respectively.

Bottom topography has the form $h(x)=h_{T} \cos k_{T} x$ with a horizontal wavenumber $k_{T}=2 \pi / 2 \mathrm{~km}^{-1}$, and an amplitude $h_{T}$ varying from $10 \mathrm{~m}$ to $80 \mathrm{~m}$. A depthindependent mean geostrophic flow $U_{G}=0.1 \mathrm{~m} \mathrm{~s}^{-1}$ is forced by adding a body force $f U_{G}$ to the meridional momentum equation. This body force represents a pressure 
gradient which balances mean flow geostrophically at all depths and is analogous to tilt of the thermocline in 2-layer model.

In order to simplify the comparison between the simulation results and the theoretical model derived in Sec. 2.4 we impose a free-slip bottom boundary condition. With this boundary condition, the theoretical model is expected to fully describe the simulations with small topographic relief when wave radiation is linear. However, since vertical resolution is small enough to resolve some of the Ekman bottom boundary layer, it would be more appropriate to use a no-slip boundary condition. To study the effect of the bottom boundary condition on wave radiation and dissipation, we run a $\mathrm{Fr}^{-1}=0.4$ simulation with both a free-slip and no-slip boundary conditions. The results are discussed in section 2.5.3 on energetics of the problem.

All experiments are initiated from a state of rest. Then, the velocity and temperature fields are slowly relaxed to the desired basic state for a 24 hour time period with a relaxation time scale of 3 hours. A gradual increase of the flow to the basic state value is necessary to let flow adjust to the bottom topography and avoid spurious initial transient effects. After the first day, the relaxation term is removed and the system is integrated for 9 more days in order to study the evolution of the wave field on geostrophic eddy time scales on the order of $L_{G} / U_{G} \sim 10$ days, where $U_{G} \approx 0.1 \mathrm{~m} \mathrm{~s}^{-1}$, and $L_{G} \approx 100 \mathrm{~km}$ have been used (Ferrari and Wunsch, 2008).

\subsubsection{Simulation results}

Numerical simulations show that there are two different regimes of wave radiation. First, at inverse Froude number lower than roughly $0.2-0.3$, waves radiate mostly in the form of lee waves, i.e steady waves tilted against mean flow. After about one day of simulation, when transient waves radiate away and lee wave front propagates throughout the domain all the way to the sponge layer, wave field is characterized by a steady monochromatic lee wave. A snapshot of the wave zonal velocity from $F r^{-1}=0.1$ simulation is shown in Fig. 2-1. The horizontal wavelength of the lee wave, $2 \mathrm{~km}$, is set by topography, and the vertical wavelength, about $600 \mathrm{~m}$, is consistent with internal wave dispersion relation. Both the slope and the amplitude of the lee 
wave are well described by linear theory. The lee wave generated at the bottom propagates all the way to the sponge layer at $2 \mathrm{~km}$ without significant changes in its amplitude. Zonally averaged flow (not shown) remains constant throughout the time of the simulation.

Second regime, at higher inverse Froude numbers, is characterized by radiation of time-dependent and multichromatic internal waves. A snapshot of the wave zonal velocity from $\mathrm{Fr}^{-1}=0.4$ simulation is shown in Fig. 2-2. Although both $F r^{-1}=0.1$ and $\mathrm{Fr}^{-1}=0.4$ simulations have the same initial conditions and external forcing and differ only by the amplitude of topography, wave radiation in $F r^{-1}=0.4$ simulation is substantially different from the classical lee wave radiation problem. Radiated waves are strongly multichromatic with vertical scales varying from the lee wave scale of about $600 \mathrm{~m}$ down to a $100 \mathrm{~m}$ scale. There is wave breaking and dissipation in the bottom several hundred meters where wave amplitudes drop down by an order of magnitude. Wave momentum deposition associated with wave breaking and dissipation feeds back on the zonally average flow. Fig. 2-3 shows the time evolution of the zonally averaged flow from $\mathrm{Fr}^{-1}=0.4$ simulation. As opposed to the low inverse Froude number regime, zonally averaged flow in highly time-dependent. Besides a constant geostrophic flow of $0.1 \mathrm{~m} \mathrm{~s}^{-1}$ (not shown) there are oscillations at inertial frequency in the bottom several hundred meters which develop spontaneously and reach a magnitude comparable to the mean flow.

Presumably, the triggering and growth of inertial frequency response in the simulations are driven by feedback between zonally averaged flow and internal waves. When inertial oscillation reaches finite amplitude it modifies wave generation and results in radiation of time-dependent and multichromatic internal waves. Vertical shear associated with inertial oscillations can modulate upward propagating internal waves and promote their braking. Before we get to the detailed analysis of the problem, we develop the theoretical model to describe the dynamics of high inverse Froude number simulations and to understand whether inertial oscillations are generated as a result of the intrinsic dynamics of the system. 


\subsection{Dynamical regimes}

The numerical simulations described above show that the radiation and breaking of internal waves triggers vigorous inertial oscillations at the ocean bottom. The development of inertial oscillations makes the problem time dependent, unlike the classical steady lee wave problem. To account for this effect we develop a weakly nonlinear analysis of wave generation by the superposition of a constant geostrophic flow and a horizontally uniform inertial oscillation.

The basic model configuration is sketched in Fig.2-4. In this idealization, the only dimensional parameters that enter in the problem are: the horizontal wavenumber of the topographic bumps, $k_{T}$, the height of the topography, $h_{T}$, the geostrophic mean velocity $U_{G}$, the amplitude of the inertial oscillation $U_{I}$, the Coriolis frequency $f$, and stratification frequency $N$. These parameters can be collapsed into three nondimensional parameters that characterize the different dynamical regimes that can develop in the problem, and the properties of the radiated waves and the associated turbulent mixing.

The first parameter, the frequency ratio $\chi$, controls whether waves are radiated from topography. The problem to be described in the next section relies on the radiation of stationary lee waves into the ocean interior. Nonlinear distortion of the lee wave pattern then triggers time dependence and feedback on the mean flow. Stationary lee waves have an intrinsic frequency given by $U_{G} k_{T}$. Hence in order for the dynamics to be described below to hold, the nondimensional parameter,

$$
\chi=\frac{U_{G} k_{T}}{N}
$$

must be in the radiative range $f / N<\chi<1$.

Second, and the most dynamically significant, nondimensional number is the

steepness parameter $\epsilon$. It is defined as the ratio of topographic slope $k_{T} h_{T}$ to the slope of the phase lines of the radiated internal waves $k_{T} / \mu$, where $\mu$ is the vertical scale of the waves,

$$
\epsilon=\mu h_{T}
$$


For steady lee waves the steepness parameter $\epsilon$ is related to the inverse Froude number,

$$
\epsilon \approx \frac{N h_{T}}{U_{G}}=F r^{-1}
$$

where we used the result that for lee waves $\mu \approx N / U_{G}$ as long as $f / N \ll \chi \ll 1$. Time dependence in the bottom velocity allows for generation of waves with different vertical structures and break the simple relationship between $\epsilon$ and $\mathrm{Fr}^{-1}$. However in our problem the time dependence is slaved to the lee wave problem and hence the inverse Froude number remains a useful parameter to characterize different dynamical regimes.

The steepness parameter, or the inverse Froude number for lee waves, measures the effect of stratification on the flow and is used to distinguish different topography regimes: sub-critical $\epsilon \ll 1$, critical $\epsilon \sim 1$, and super-critical $\epsilon \gg 1$. In the first regime, sub-critical topography, stratification has a weak effect on wave generation and an internal wave field sets up above the topography, transporting momentum and energy upward into the ocean interior. In this limit, the lower boundary condition can be linearized and wave solutions are given by linear theory. In the critical topography regime, nonlinear effects become important and waves start breaking above topography. In the super-critical regime, stratification is large enough to suppress vertical motions and radiation of waves. Basically the mean flow does not have enough kinetic energy to overcome the stratification and go over the sill. The mean flow is therefore deflected and goes around, rather than over, the topography. 2-D models are not appropriate to describe super-critical flows, because the flow cannot go around the topography and is therefore blocked by topography.

The frequency of the waves radiated by a time dependent flow is controlled by a third parameter, the excursion parameter,

$$
\beta=\frac{U k_{T}}{\omega}
$$

where $\omega$ is the frequency of oscillation of the time-dependent flow and $U$ is its amplitude. This number compares the amplitude of particle excursion $U \omega^{-1}$ to the 
horizontal scale of the topographic bumps, $k_{T}^{-1}$. There are three dynamical regimes which are classified by the excursion parameter. For $\beta \ll 1$, the particle excursion is less than the scale of topography. The waves radiate mainly at the fundamental frequency $\omega$ in both upstream and downstream directions. The interaction of a barotropic tide with large-scale bottom topography falls into this dynamical regime. For $\beta \sim 1$, the particle excursion is comparable with the scale of topography. In this regime, advection of the waves by the mean oscillating flow becomes important and results in the generation of waves with frequency higher than $\omega$, making the wave field non-monochromatic. For $\beta \gg 1$, one recovers the quasi-steady lee wave regime with waves propagating only in the upstream direction.

Here, we consider a flow which is the superposition of a time-independent flow of amplitude $U_{G}$ and an inertial oscillation of an amplitude $U_{I}$ and frequency $f$. In this case the frequency of the inertial oscillation is the characteristic frequency of the time-dependent flow and the particle excursion associated with this oscillation determines the wave response of the system. Then, the relevant excursion parameter is,

$$
\beta=\frac{U_{I} k_{T}}{f}
$$

It is useful to estimate the three nondimensional numbers for the flows observed in the Drake Passage region used here as a prototype situation for the idealized problem. With Drake Passage parameters we have, $\epsilon \sim 0.6, \chi \sim 0.3$. Both numerical simulations and theory described below suggest that inertial oscillations reach the same amplitude as the mean geostrophic flow, $U_{I} \approx U_{G}$, corresponding to $\beta \sim 3$. Hence radiation of internal waves in Drake Passage is close to critical, waves can be radiated, and advective effects are large enough to generate harmonics beyond the fundamental frequency $f$.

\subsection{Theory}

A number of analytical models of topographic internal wave generation have been developed for both internal tides and lee waves. Ocean models have been mostly used 
to predict the conversion of energy from barotropic tides into the internal waves, while the atmospheric literature focuses on the steady lee wave problem. The linear approach for the ocean was developed by Bell $(1975 \mathrm{a}, \mathrm{b})$. Bell considered a barotropic current flowing over topography in a vertically unbounded ocean with uniform stratification. He restricted the analysis to small topographic slopes $(\epsilon \ll 1)$ so that topography was everywhere sub-critical and the bottom boundary condition could be linearized. With this simplification, solutions for arbitrary topography can be obtained by superposition. Bell's theory can be applied to study both the internal tide and quasi-steady lee wave regimes.

Bell's model has two major limitations when applied to the tidal problem: (1) the vertical scale of the radiated internal tides is typically of the same order of the ocean depth and the assumption of infinite depth (and uniform stratification) is not tenable; (2) topographic slopes can be quite steep, i.e. $\epsilon$ is not necessarily small. Llewellyn-Smith and Young (2002) and Khatiwala (2003) showed that when a rigid lid is imposed at the ocean top, radiation by topographic scales longer than the horizontal wavelength of the first mode is suppressed. Balmforth et al. (2002) and St.-Laurent and Garrett (2002) found that finite amplitude corrections to the energy flux are small as long as topography remains sub-critical $(\epsilon \leq 1)$.

The assumption of infinite depth is not a major issue for internal waves generated by subinertial and inertial flows, a regime we will refer to as that of time-dependent lee waves. Unlike internal tides, these waves are radiated with vertical scales shorter than the scale of the first mode and their generation is not directly affected by the surface boundary condition. The assumption of sub-critical topography is more questionable because time-dependent lee waves are generated by small-scale topographic features which can be quite steep. We will therefore compare the results of linear theory, valid for small $\epsilon$, with numerical simulations in the finite steepness parameter limit. Finally no assumption of small $\beta$ will be made (as generally done in tidal studies, e.g. Llewellyn-Smith and Young (2002); Balmforth et al. (2002)) because time dependent oscillations tend to become as large as the mean geostrophic flow, i.e. $\beta=O(1)$. 


\subsubsection{Problem formulation}

The major contribution of this work is to study the response of the mean flow to topographic radiation of internal waves. We start by deriving the equations that describe the evolution of the zonally averaged flow on a slow time scale and the internal waves on a fast time scale. The scale separation is achieved by expanding equations and boundary conditions in the steepness parameter $\epsilon$. We idealize the ocean as a Boussinesq, rotating, and stably stratified fluid governed by

$$
\begin{aligned}
\mathbf{u}_{t}+\left(\mathbf{u} \cdot \nabla_{H}\right) \mathbf{u}+w \mathbf{u}_{z}+f \hat{\mathbf{z}} \times \mathbf{u} & =-\nabla_{H} p+\nu \nabla^{2} \mathbf{u}, \\
w_{t}+\left(\mathbf{u} \cdot \nabla_{H}\right) w+w w_{z} & =-p_{z}+b+\nu \nabla^{2} w \\
b_{t}+\left(\mathbf{u} \cdot \nabla_{H}\right) b+w b_{z}+w N^{2} & =\kappa \nabla^{2} b, \\
\nabla_{H} \cdot \mathbf{u}+w_{z} & =0,
\end{aligned}
$$

where $\mathbf{u}=(u, v)$ is the horizontal velocity vector, $w$ the vertical velocity, $b=-g \rho / \rho_{0}$ buoyancy, $p$ pressure, $f$ the Coriolis parameter, $N$ the buoyancy frequency, $\nu$ the viscosity, and $\kappa$ the diffusivity.

The flow is assumed to be periodic in the horizontal. Top and bottom boundary conditions are of vanishing vertical velocity as $z \rightarrow \infty$ and zero velocity normal to topography,

$$
\begin{aligned}
& w=0, \quad \text { at } z \rightarrow \infty, \\
& w=\mathbf{u} \cdot \nabla_{H} h(\mathbf{x}), \quad \text { at } z=h(\mathbf{x}) .
\end{aligned}
$$

First we nondimensionalize both the governing equations and the boundary condition (see Appendix A). Next, we expand the solution into a series of a small steepness parameter $\epsilon$ and impose that to the leading order there is a superposition of a geostrophic flow and an inertial oscillation,

$$
\mathbf{u}=\mathbf{u}^{G}\left(T_{G}, \mathbf{X}_{G}, Z_{G}\right)+\mathbf{u}^{I}\left(t ; T_{I}, \mathbf{X}_{I}, Z_{I}\right)+\epsilon \mathbf{u}^{(1)}+\epsilon^{2} \mathbf{u}^{(2)}+\epsilon^{3} \mathbf{u}^{(3)}+\ldots
$$


where $\mathbf{u}^{G}$ is the geostrophic flow evolving on a slow time scale $T_{G}$ and large spatial scales $\mathbf{X}_{G}$ and $Z_{G}, \mathbf{u}^{I}$ is an inertial oscillation which oscillates at frequency $f$ on a fast time scale $t$ and can also evolve on a slow time scale $T_{I}$ and large spatial scales $\mathbf{X}_{I}$ and $Z_{I}$, and $\mathbf{u}^{(n)}$ are the higher order motions that depend on all scales of the problem. Details of the expansion are given in Appendix B. At leading order, $O\left(\epsilon^{0}\right)$, we obtain a set of equations describing both geostrophic,

$$
f \hat{\mathbf{z}} \times \mathbf{u}^{G}=-\nabla_{\mathbf{X}_{G}} p^{G}, \quad 0=-p_{Z_{G}}^{G}+b^{G}, \quad w^{G}=0,
$$

and inertial oscillation components of the mean flow,

$$
\mathbf{u}_{t}^{I}+f \hat{\mathbf{z}} \times \mathbf{u}^{I}=0, \quad w^{I}=0
$$

At the following order, $O\left(\epsilon^{1}\right)$, we get a set of equations and the bottom boundary condition governing fast time scale evolution of internal waves generated by the leading order motions interacting with topography,

$$
\begin{aligned}
\mathbf{u}_{t}^{(1)}+\left(\mathbf{u}^{G}+\mathbf{u}^{I}\right) \cdot \nabla_{H} \mathbf{u}^{(1)}+f \hat{\mathbf{z}} \times \mathbf{u}^{(1)} & =-\nabla_{H} p^{(1)}+\nu \nabla^{2} \mathbf{u}^{(1)} \\
w_{t}^{(1)}+\left(\mathbf{u}^{G}+\mathbf{u}^{I}\right) \cdot \nabla_{H} w^{(1)} & =-p_{z}^{(1)}+b^{(1)}+\nu \nabla^{2} w^{(1)} \\
b_{t}^{(1)}+\left(\mathbf{u}^{G}+\mathbf{u}^{I}\right) \cdot \nabla_{H} b^{(1)}+N^{2} w^{(1)} & =\kappa \nabla^{2} b^{(1)} \\
\nabla_{H} \cdot \mathbf{u}^{(1)}+w_{z}^{(1)} & =0 \\
\left.w^{(1)}\right|_{z=0} & =\left(\mathbf{u}^{G}+\mathbf{u}^{I}\right) \cdot \nabla_{H} h .
\end{aligned}
$$

Finally, the evolution of the oscillating component of the mean flow on the slow time $T_{I}$ and large spatial scales $\left(\mathbf{X}_{I}, Z_{I}\right)$ is described by the $O\left(\epsilon^{3}\right)$ equations,

$$
\begin{aligned}
\overline{\mathbf{u}_{t}^{(3)}}+f \hat{\mathbf{z}} \times \overline{\mathbf{u}^{(3)}} & =-\mathbf{u}_{T_{I}}^{I}-\partial_{Z_{I}} \overline{w^{(1)} \mathbf{u}^{(1)}} \\
0 & =-\partial_{Z_{I}} \overline{w^{(1)} w^{(1)}}-P_{Z_{I}}^{I}+b^{I}, \\
b_{t}^{I} & =-\partial_{Z_{I}} \overline{w^{(1)} b^{(1)}}
\end{aligned}
$$


where the over-bar represents an average over small spatial scales. At this order, there is a fast time evolution of the $O\left(\epsilon^{3}\right)$ inertial oscillation combined with a slow time evolution of the leading order inertial oscillation $\mathbf{u}^{I}$ forced by $O\left(\epsilon^{1}\right)$ internal wave momentum flux divergence. The evolution of the geostrophic mean flow component is assumed to occur on a slower time scale $T_{G} \gg T_{I}$ and larger spatial scales $\left(\mathbf{X}_{G}, Z_{G}\right) \gg$ $\left(\mathbf{X}_{I}, Z_{I}\right)$, i.e. we assume a scale separation between geostrophic motions and inertial oscillations, a reasonable approximation as long as $R o \ll 1$ (see Appendix B). Under this approximation the evolution of the geostrophic motions enter at orders higher than $O\left(\epsilon^{3}\right)$.

\subsection{2 $O\left(\epsilon^{0}\right)$ solution: mean flow}

The zonally-averaged flow is given by the solution of the $O\left(\epsilon^{0}\right)$ equations,

$$
\begin{aligned}
& \bar{u}(t)=U_{G}+U_{I} \cos f\left(t-t_{0}\right), \\
& \bar{v}(t)=-U_{I} \sin f\left(t-t_{0}\right),
\end{aligned}
$$

where $U_{G}$ is a zonal geostrophic flow governed by equations (2.13) varying on the very slow variables $\left(\mathbf{X}_{G}, T_{G}\right), U_{I}$ and $f t_{0}$ are the amplitude and phase of inertial oscillation governed by equations (2.14) varying on the slow variables $\left(\mathbf{X}_{I}, T_{I}\right)$.

\subsection{3 $O\left(\epsilon^{1}\right)$ solution: wave radiation}

We now solve the internal wave generation problem at $O\left(\epsilon^{1}\right)$ for a $2-\mathrm{D},(x, z)$, idealized mean flow (2.23) and (2.24) over one-dimensional, monochromatic bottom topography. To make analytical progress we simplify dissipation terms and make change of reference frame to the system moving with the mean flow.

The bottom topography is given by,

$$
h(x)=h_{T} \cos \left(k_{T} x\right),
$$

where $h_{T}$ is the topography amplitude, and $k_{T}$ is its horizontal wavenumber. Flows 
over bottom topography generate internal waves radiating upward into the ocean interior. The dynamics of internal waves is governed by the set of equations (2.15)(2.18) with the lower boundary condition (2.19). To simplify the linear analysis we set

$$
\nu \nabla^{2} \mathbf{u}=-\lambda \mathbf{u}^{(1)}, \quad \nu \nabla^{2} w=-\lambda w^{(1)}, \quad \kappa \nabla^{2} b=-\lambda b^{(1)},
$$

where $\lambda$ is the Rayleigh damping rate. Using Rayleigh damping instead of the harmonic scale-selective friction and dissipation captures the effect of wave dissipation while keeping the algebra much simpler. In the numerical simulations described below, we use harmonic dissipation, but damping is mostly due to turbulent dissipation resulting from breaking of the waves. It is not clear whether wave breaking is best described by linear or harmonic dissipation. What matters is that both forms capture the damping of buoyancy and momentum fluctuations resulting from breaking.

In order to find an analytical solution to the problem, it is convenient to change the reference frame to the coordinate system moving with the zonally averaged flow $\bar{u}(t)$,

$$
\xi=x-\int_{t_{0}}^{t} \bar{u}(t) d t=x-U_{G}\left(t-t_{0}\right)-\frac{U_{I}}{f} \sin f\left(t-t_{0}\right) .
$$

In the moving reference frame, the dynamics in (2.15)-(2.18) can be reduced to a single equation for the vertical velocity $w^{(1)}$,

$$
\left(\partial_{t t}+2 \lambda \partial_{t}+\lambda^{2}\right) \nabla^{2} w^{(1)}+N^{2} w_{\xi \xi}^{(1)}+f^{2} w_{z z}^{(1)}=0
$$

Periodic topography implies a periodic solution, and we can expand the solution into Fourier modes in the $\xi$-coordinate frame. Using a Jacobi-Anger expansion, the lower boundary condition can be written as a superposition of plane waves,

$$
\left.\tilde{w}^{(1)}\right|_{z=0}=i \hat{h} \sum_{n=-\infty}^{\infty} \sigma_{n} J_{n}(\beta) e^{i \sigma_{n}\left(t-t_{0}\right)}
$$

where $\sigma_{n}=n f+U_{G} k$ is the intrinsic frequency of the $n$-th harmonic of the inertial frequency Doppler shifted by the mean geostrophic flow. The excursion parameter $\beta$ is defined in $(2.5)$, and $J_{n}(\beta)$ are the Bessel functions of the first kind. For $\beta$ 
finite, $n$ can be different from zero and waves are radiated at super-harmonics of $f$. The horizontal structure and time dependence of the internal wave solution in the interior are dictated by the lower boundary condition (2.29): the solution is a superposition of waves not only at the fundamental frequency $f$, but also at all of its harmonics. The energy distribution over different harmonics depends on the value of excursion parameter $\beta$. The larger the excursion parameter, the more energy is in higher harmonics. The fact that the internal wave field is a superposition of inertial frequency harmonics has important implications for the wave-mean flow feedback mechanism discussed later in the next section.

The solution to (2.28) satisfying boundary condition (2.29) and evaluated for a particular form of topography (2.25) is a superposition of a number of harmonics each taking the form,

$$
w_{n}^{(1)}(t, \xi, z)=-h_{T} \sigma_{n} J_{n}(\beta) \Im\left[e^{i \theta_{n}}\right], \quad \theta_{n}=k_{T} \xi+\mu_{n} z+\sigma_{n}\left(t-t_{0}\right)
$$

where $\mu_{n}$ is a complex number with the real and imaginary parts representing, respectively, the wave vertical wavenumber and an inverse decay scale due to damping,

$$
\mu_{n}^{2}=k_{T}^{2} \frac{N^{2}-\left(\sigma_{n}-i \lambda\right)^{2}}{\left(\sigma_{n}-i \lambda\right)^{2}-f^{2}}
$$

Solutions for other wave variables can be easily found from equations (2.15)-(2.18) in the moving reference frame using (2.30),

$$
\begin{aligned}
u_{n}^{(1)}(t, \xi, z) & =h_{T} \sigma_{n} J_{n}(\beta) \Im\left[\frac{\mu_{n}}{k_{T}} e^{i \theta_{n}}\right] \\
v_{n}^{(1)}(t, \xi, z) & =h_{T} \sigma_{n} J_{n}(\beta) \Re\left[\frac{\mu_{n}}{k_{T}} \frac{f}{\left(\sigma_{n}-i \lambda\right)} e^{i \theta_{n}}\right], \\
p_{n}^{(1)}(t, \xi, z) & =h_{T} \sigma_{n}^{2} k_{T}^{-1} J_{n}(\beta) \Im\left[\frac{\mu_{n}}{k_{T}} \frac{\left(\sigma_{n}-i \lambda\right)^{2}-f^{2}}{\sigma_{n}\left(\sigma_{n}-i \lambda\right)} e^{i \theta_{n}}\right] .
\end{aligned}
$$

where $\Re$ and $\Im$ are the real and imaginary parts.

The bottom energy conversion rate from subinertial and inertial flows into internal waves averaged zonally and over an inertial period can be calculated using (2.30) and 
(2.34) as,

$$
\overline{w^{(1)} p^{(1)}}{ }^{x, t}=\frac{1}{2} \sum_{n=-\infty}^{\infty} h_{T}^{2} \sigma_{n}^{3} k_{T}^{-1} J_{n}^{2}(\beta) \cdot \Re\left[\frac{\mu_{n}}{k_{T}} \frac{\left(\sigma_{n}-i \lambda\right)^{2}-f^{2}}{\sigma_{n}\left(\sigma_{n}-i \lambda\right)}\right] .
$$

This expression reduces to the well known expressions for lee wave radiation in the limit of a zero inertial oscillation amplitude or to the expression for internal tide conversion, if the tidal frequency is used instead of inertial frequency (Bell, 1975a,b). If the excursion paramter is small then the internal wave energy flux is proportional to the topographic slope. In the large excursion parameter limit, however, the energy flux is no longer proportional to the slope, but rather to the amplitude of topography (Polzin, 2004).

\subsection{4 $O\left(\epsilon^{3}\right)$ solution: feedback of waves on mean flow}

Here, we consider the feedback of internal waves on the mean flow. We solve $O\left(\epsilon^{3}\right)$ equations (equations at $O\left(\epsilon^{2}\right)$ are trivial as shown in Appendix B), which describe slow-time evolution of the $O\left(\epsilon^{0}\right)$ inertial oscillation driven by the divergence of the $O(\epsilon)$ wave momentum fluxes. Using the complex velocity form $\gamma=u+i v,(2.20)$ can be written as,

$$
\overline{\gamma_{t}^{(3)}}+i f \overline{\gamma^{(3)}}=-\gamma_{T_{I}}^{I}-\partial_{z} \overline{w^{(1)} \gamma^{(1)}}
$$

where $\gamma_{T_{I}}^{I}$ represents variation of the leading order inertial oscillation on the slow timescale $T_{I}=\epsilon^{3} t$. There is no forcing of the geostrophic mode at this order because we assumed that the geostrophic flow varies on temporal and spatial scales longer than $T_{I}=\epsilon^{3} t$ and $Z_{I}=\epsilon z$ respectively which requires that its Rossby number is smaller than $O\left(\epsilon^{3}\right)$.

In order to solve (2.36), we plug in both the momentum flux divergences computed from internal wave solutions $(2.30),(2.32)$ and $(2.33)$ and the $O\left(\epsilon^{0}\right)$ solution for $\gamma^{I}$, from equation (2.14), which is given by,

$$
\gamma^{I}\left(t, T_{I}\right)=U_{I}\left(T_{I}\right) e^{-i f\left(t-t_{0}\left(T_{I}\right)\right)}
$$


where the inertial oscillation amplitude, $U_{I}$, and phase, $-f t_{0}$, can evolve on the slow time scale $T_{I}$.

We are particularly interested in the growth of inertial oscillations at the bottom because they can feedback on the wave generation. The momentum flux divergence at $z=0$ is,

$$
\partial_{z} \overline{w^{(1)} \gamma^{(1)}}=\frac{1}{4} h_{T}^{2} k_{T}^{-1} \sum_{m=-\infty}^{\infty} \sum_{n=-\infty}^{\infty} \sigma_{m} \sigma_{n} J_{m}(\beta) J_{n}(\beta)\left(a_{m n} e^{i \theta_{m n}}+b_{m n} e^{-i \theta_{m n}}\right),
$$

where coefficients $a_{m n}$ and $b_{m n}$ are given by,

$$
\begin{aligned}
a_{m n} & =-i\left(1-\frac{f}{\sigma_{n}-i \lambda}\right) \mu_{n}\left(\mu_{n}-\mu_{m}^{*}\right), \\
b_{m n} & =i\left(1+\frac{f}{\sigma_{n}-i \lambda}\right)^{*} \mu_{n}^{*}\left(\mu_{n}^{*}-\mu_{m}\right),
\end{aligned}
$$

and $\theta_{m n}$ is the phase of the flux formed by two wave harmonics $m$ and $n$ and evaluated at $z=0$, i.e. $\left.\theta_{m n}\right|_{z=0}=\left.\left(\theta_{n}-\theta_{m}\right)\right|_{z=0}=\left(\sigma_{n}-\sigma_{m}\right)\left(t-t_{0}\right)=(n-m) f\left(t-t_{0}\right)$. The flux divergence has a steady component $(m=n)$. But it also has a component oscillating at the inertial frequency for $m=n \pm 1$, which can trigger a resonant response and growth of inertial oscillation. Whether a resonance develops or not depends on the relative orientation of the force due to the momentum fluxes $-\partial_{z} \overline{w^{(1)} \mathbf{u}^{(1)}}$ and the velocity $\mathbf{u}^{I}$ (Fig. 2-5). If waves are inviscid $(\lambda=0)$, then $a_{m n}$ and $b_{m n}$ are imaginary and the flux divergence averaged over an inertial period is in quadrature with $\mathbf{u}^{I}$ (i.e. the accelerations and decelerations due to $-\partial_{z} \overline{w^{(1)} \mathbf{u}^{(1)}}$ average to zero over an inertial period). If there is wave dissipation, however, a net force develops over a period that acts to accelerate the inertial oscillation and drive a resonance.

To understand how the resonant mechanism can drive instability of inertial oscillation let us consider the case when the amplitude of the inertial oscillation is small, i.e. $\beta \ll 1$. This assumption allows us to expand flux divergence (2.38) for small $\beta$. At the leading order, $O\left(\beta^{0}\right)$, there is a steady momentum flux divergence associated 
with the damped, $n=0$, stationary harmonic,

$$
\partial_{z} \overline{w^{(1)} \gamma^{(1)}}=\frac{1}{4} h_{T}^{2} k_{T}^{-1} \sigma_{0}^{2}\left(a_{0,0}+b_{0,0}\right)
$$

At the following order, $O\left(\beta^{1}\right)$, the flux divergence oscillates at the resonant frequency $f$ and is given by,

$$
\partial_{z} \overline{w^{(1)} \gamma^{(1)}}=-f U_{I}\left(A e^{i f\left(t-t_{0}\right)}+B e^{-i f\left(t-t_{0}\right)}\right)
$$

where coefficients $A$ and $B$ depend on properties of wave modes -1,0, and 1 and defined as,

$$
\begin{aligned}
A & =\frac{1}{8} \frac{h_{T}^{2} \sigma_{0}}{f^{2}}\left[\sigma_{-1}\left(a_{-1,0}+b_{0,-1}\right)-\sigma_{1}\left(a_{0,1}+b_{1,0}\right)\right] \\
B & =\frac{1}{8} \frac{h_{T}^{2} \sigma_{0}}{f^{2}}\left[\sigma_{-1}\left(a_{0,-1}+b_{-1,0}\right)-\sigma_{1}\left(a_{1,0}+b_{0,1}\right)\right]
\end{aligned}
$$

Combining (2.36), (2.37), and (2.42) we obtain,

$$
\overline{\gamma_{t}^{(3)}}+i f \overline{\gamma^{(3)}}=\left(-\dot{U}_{I}-i f U_{I} \dot{t_{0}}+f U_{I} B\right) e^{-i f\left(t-t_{0}\right)}+f U_{I} A e^{i f\left(t-t_{0}\right)}
$$

The solutions for $U^{I}\left(T_{I}\right)$ and $t_{0}\left(T_{I}\right)$ are obtained by eliminating the secular term,

$$
\begin{aligned}
U_{I}\left(T_{I}\right) & =U_{I}(0) e^{f \Re(B) T_{I}}, \\
t_{0}\left(T_{I}\right) & =\Im(B) T_{I} .
\end{aligned}
$$

We can see that, while the phase of inertial oscillation, $t_{0}\left(T_{I}\right)$, forced by the breaking waves changes linearly, the amplitude, $U_{I}\left(T_{I}\right)$, can evolve exponentially in time. A small initial perturbation $U_{I}(0)$ at inertial frequency can either grow or decay depending on the sign of the growth rate $\Gamma$, given by,

$$
\Gamma=f \Re(B) .
$$


where the coefficient $B$, given by (2.44), depends on other parameters of the problem and can be simplified to,

$$
\Gamma \simeq \frac{1}{2} \lambda F r^{-2}\left[1+4 \frac{f^{2}}{U_{G}^{2} k_{T}^{2}}-3 \frac{\lambda^{2}}{U_{G}^{2} k_{T}^{2}}\right]
$$

in the limit of,

$$
\frac{U_{G} k_{T}}{N} \ll 1, \quad \frac{f}{U_{G} k_{T}} \ll 1, \quad \frac{\lambda}{U_{G} k_{T}} \ll 1 .
$$

This is a limit where stationary lee waves radiating from topography with frequency $\omega=U_{G} k_{T}$ have a frequency larger than the inertial frequency, $\omega \gg f$, are hydrostatic $\omega \ll N$, and weakly damped $\lambda \ll \omega$. In this limit, the linear stability analysis of the wave-mean flow interaction shows that the system of a geostrophic flow over bottom topography can be unstable to a small inertial frequency perturbation in the zonally averaged flow. The growth rate of the instability, to leading order, is proportional to the squared inverse Froude number $\mathrm{Fr}^{-2}$ and the Rayleigh damping time scale $\lambda$ (representing the internal wave breaking process). The instability develops as soon as there is some damping acting on the waves, allowing for wave momentum deposition and feedback on the zonally averaged flow. However, when the damping rate $\lambda$ exceeds a certain value, greater than $f$, the growth rate changes sign and the waves start to act against inertial oscillation.

The presence of the damping is essential for the resonant feedback to occur. If there is no damping then the amplitude of the wave momentum fluxes is vertically uniform. Momentum fluxes, and their vertical divergences, are in phase with the inertial oscillation at every level and the net work over a period is zero. This result is consistent with the non-acceleration theorem. When there is damping, however, the amplitude of the wave momentum fluxes decays with height resulting in the wave momentum deposition and the phase shift between inertial oscillation and the wave momentum flux divergence. When slightly out-of-phase, the wave momentum fluxes work to accelerate inertial oscillation. However, wave momentum deposition can start to work against inertial oscillations when the phase shift becomes too large. 


\subsection{Results}

In this section we use the theory described above to interpret the results of the numerical simulations. We decompose the model solution into a zonal mean (the mean) and deviations from the zonal mean (the waves). First, we discuss the evolution of the mean flow and demonstrate that the growth of inertial oscillations in the simulations is consistent with the resonant feedback mechanism described in Sec. 2.4. Next, we compare the internal wave flux from simulations with the theoretical predictions. Finally, we discuss the energy budget of wave radiation, breaking, and dissipation and show the relevance of our results for turbulent dissipation estimates in the Southern Ocean.

\subsubsection{Inertial oscillations}

The time evolution of the mean flow from a simulation with $\mathrm{Fr}^{-1}=0.4$ is shown in Fig.2-3. No inertial oscillation is imposed in the initial condition of the simulation. However, within the first 48 hours, a strong oscillatory flow with frequency $f$ develops on top of the prescribed geostrophic mean flow of $0.1 \mathrm{~m} \mathrm{~s}^{-1}$ (not shown on the figure). The amplitude of the inertial oscillation grows rapidly in time until it reaches the same magnitude of the mean geostrophic flow and then equilibrates. The oscillations extend throughout the whole domain, but they are particularly intense within $700 \mathrm{~m}$ of the bottom.

The growth of the inertial oscillations at $z=100 \mathrm{~m}$, slightly above the topography, is shown in Fig. 2-6 for the whole set of simulations. The growth rate of the amplitude of the oscillations increases with inverse Froude number. For $\mathrm{Fr}^{-1} \leq 0.3$, the growth rate is very slow and it has not reached an equilibrium after 10 days of simulation. Simulations with $\mathrm{Fr}^{-1}>0.3$ have a fully developed inertial oscillations with

amplitude of about $0.12 \pm 0.02 \mathrm{~ms}^{-1}$, i.e. of the same order as the mean geostrophic flow.

Although the scale separation between inertial oscillations and internal waves in the vertical assumed in linear theory is not well satisfied in the simulations, the 
characteristics of the inertial oscillations are consistent with the resonant feedback mechanism described in Sec. 2.4. The resonant mechanism predicts that the vertical structure of the inertial oscillation is set by the divergence of internal wave momentum flux oscillating at frequency $f$. For the reference simulation shown in Fig.2-3, the wave momentum flux is dominated by harmonics -1 and 0 and it has a vertical scale of $2 \pi\left(\mu_{-1}^{r}-\mu_{0}^{r}\right)^{-1} \sim 1 \mathrm{~km}$ : the phase lines of the inertial oscillations in $(t, z)$ space have indeed a slope very close to $f /\left(\mu_{-1}^{r}-\mu_{0}^{r}\right)$.

For $\mathrm{Fr}^{-1}>0.3$, waves break above topography and deposit most of their momentum before reaching the sponge layer. The inertial oscillations are most pronounced in regions where wave breaking occurs. For the reference simulation, $\mathrm{Fr}^{-1}=0.4$, the vertical extent of the region over which the momentum flux decays from its bottom value to approximately zero is $700 \mathrm{~m}$.

In the theoretical model of Sec. 2.4 we represented wave breaking as a Rayleigh damping process. A Rayleigh damping of $\lambda=5 \times 10^{-5} \mathrm{~s}^{-1}$ reproduces the vertical decay scale of the time-averaged internal wave momentum flux for the reference simulation. We discuss what sets this scale below. Here, we want to show that linear theory can be used to relate the scale of wave breaking, through $\lambda$, to various properties of the inertial oscillations. The evolution of inertial oscillation from simulation and theory are compared in Fig. 2-7. The theoretical prediction is obtained by integrating eq. (2.36) forced with the wave momentum flux divergence in (2.38) which depends on $\lambda$. The theoretical model reproduces remarkably well both the initial growth and the final amplitude saturation phases. It slightly overestimates the magnitude at which the inertial oscillations saturate because linear theory does not account for their dissipation. The small- $\beta$ expression for the initial growth rate of inertial oscillation is also very accurate at early times and further builds our confidence that the resonant generation mechanism is key to the appearance of inertial oscillations. This comparison holds for the other inverse Froude number simulations as well. 


\subsubsection{Wave radiation}

We now test the prediction of linear theory for the energy flux radiated by topographic internal waves. We estimate the energy flux $\overline{p^{\prime} w^{\prime}}$ from the model solution using deviations from the zonal mean and averaging the flux zonally in space and over several inertial periods in time. Fig. 2-8 shows the vertical dependence of the energy flux for different Froude number simulations. The value of the energy flux radiated from the bottom increases with inverse Froude number as the wave amplitude increases and waves can transport more energy. The bulk of the energy flux decays substantially within less than $1 \mathrm{~km}$ of the bottom, as a result of wave breaking and dissipation. Above the breaking level there is a small residual energy flux that radiates into the ocean interior.

Fig. 2-9 shows the comparison of the bottom value of the energy flux between numerical simulations and the linear theory prediction. To make theoretical prediction, we use the inertial oscillation amplitude diagnosed from the simulations in (2.35), i.e. we set $U_{I}=0.12 \mathrm{~m} \mathrm{~s}^{-1}$. The results indicate that the presence of inertial oscillations in the mean flow over bottom topography increases the amount of energy radiated by internal waves by about $30 \%$ compared to the lee wave radiation estimate. The effect of inertial oscillations on wave generation is well captured by the linear wave generation theory, which includes radiation of inertial frequency harmonics.

The characteristics of the radiated waves change substantially as a function of $\mathrm{Fr}^{-1}$ and can be described by three different regimes. The first regime, at small inverse Froude number (smaller than 0.3 in our simulations), is characterized by stationary lee wave generation. Inertial oscillation do grow in time as a result of the resonant feedback, but the growth rate is small and over 10 days they do not develop enough to significantly modify the wave generation process. In this regime, wave generation is well described by the linear lee wave generation theory. A second regime develops for $0.3<F r^{-1}<0.7$, where inertial frequency harmonics are generated. In this Froude number range, inertial frequency perturbations in the mean flow grow rapidly and reach an amplitude comparable with that of the mean flow within several 
days. These inertial oscillations significantly modify the wave generation process by not only increasing the amount of radiated energy but also by making the wave field substantially time dependent and non-monochromatic. Linear wave generation theory which includes inertial oscillations in the mean flow agrees well with numerical simulations in this Froude number range. The last regime, $\mathrm{Fr}^{-1}>0.7$, is the wave saturation regime. Internal wave radiation saturates, i.e. it does not increase anymore with the inverse Froude number. This can be explained by the blocking effect which topography has on the mean flow. At a certain topography amplitude, flow from the deep valley does not have enough kinetic energy to go over the bump and is blocked by topography. Only some fraction of the flow which is close to the top of the bump can go over it and generate internal waves. In this regime further increasing the topographic height does not allow more flow to be deflected, and the wave radiation does not grow.

While regime one and three have been studied extensively, regime two has not been the focus of much investigation. Here we focus on it, because it turns out to be quite relevant for the oceanic situation we are interested in. The intermediate Froude number range, $0.3<F r^{-1}<0.7$, is characterized by the radiation of inertial frequency harmonics. Although the linear theory solution is a superposition of an infinite number of harmonics, only those that satisfy $f<\sigma_{n}<N$ will radiate into the ocean interior and contribute to the value of the energy flux. Moreover, the distribution of energy over different harmonics depends on the value of the excursion parameter $\beta$. For instance, during the onset of instability, when the inertial oscillation amplitude is small, most of the energy is in the lower, $n=0, \pm 1$, harmonics. As shown in the resonant feedback section, this set of wave modes is enough to feedback on the mean flow at the inertial frequency, the resonant frequency of the system. As the inertial oscillation amplitude grows, the excursion parameter $\beta$ gets larger, and higher harmonics radiate. 


\subsubsection{Energy pathways}

A substantial fraction of the energy radiated by gravity waves is dissipated within $1 \mathrm{~km}$ of the bottom topography in the simulations presented so far. This layer is characterized by vigorous turbulence, especially for higher $\mathrm{Fr}^{-1}$ simulations, $\mathrm{Fr}^{-1}>$ 0.3 , resulting from breaking of internal waves (Fig. 2-2). To understand the pathways of energy from the prescribed mean geostrophic flow to turbulent dissipation at small scales associated with internal wave breaking, we estimate the mean and wave kinetic energy budgets for the layer $1 \mathrm{~km}$ above the bottom. We decompose the model solution into the mean and waves as follows,

$$
\mathbf{u}=U_{0} \hat{\mathbf{i}}+\overline{\mathbf{u}}+\mathbf{u}^{\prime}
$$

where $U_{0}$ is the prescribed geostrophic flow, $\overline{\mathbf{u}}$ is the zonal mean flow, and $\mathbf{u}^{\prime}$ represents wave perturbations. Generally, $\overline{\mathbf{u}}$ includes both inertial and subinertial flow components. However, the subinertial flow does not change much over the simulated period of time. Since the domain of simulation is two-dimensional and zonally periodic, no pressure gradient can develop to balance changes in the subinertial flow. The zonal and time mean flow therefore remains the same throughout the simulation and a weak, about 3-4 $\mathrm{mm} \mathrm{s}^{-1}$, meridional mean flow is driven by the divergence of the wave momentum fluxes (Fig. 2-10). Thus, $\overline{\mathbf{u}}$ is dominated by inertial oscillations.

The kinetic energy equation for the zonal mean flow $\overline{\mathbf{u}}$ averaged in time over many inertial periods takes the form,

$$
\partial_{t} M K E-\nu \partial_{z z} M K E=-<\overline{\mathbf{u}} \cdot \partial_{z} \overline{w^{\prime} \mathbf{u}^{\prime}}>-\epsilon_{M K E},
$$

where $M K E=\frac{1}{2}<\overline{\mathbf{u}} \cdot \overline{\mathbf{u}}>$ is the kinetic energy of the mean flow, and terms on the right hand side are the conversion of energy between the mean flow and the waves and the mean flow dissipation $\epsilon_{M K E}=\nu<\left|\overline{\mathbf{u}}_{z}\right|^{2}>$ respectively. The second term on the left is the transport of the mean kinetic energy by viscous terms. Overline and brackets represent spatial and time averages, respectively. 
Similarly, the kinetic energy equation for the wave component $\mathbf{u}^{\prime}$ can be written as,

$$
\begin{array}{r}
\partial_{t} E K E-\nu \partial_{z z} E K E= \\
-\partial_{z}\left[<\overline{p^{\prime} w^{\prime}}>+U_{0}<\overline{w^{\prime} u^{\prime}}>+<\overline{\mathbf{u}} \cdot \overline{w^{\prime} \mathbf{u}^{\prime}}>+\frac{1}{2}<\overline{w^{\prime} \mathbf{u}^{\prime} \cdot \mathbf{u}^{\prime}}>\right]+ \\
+U_{0} \partial_{z}<\overline{w^{\prime} u^{\prime}}>+<\overline{\mathbf{u}} \cdot \partial_{z} \overline{w^{\prime} \mathbf{u}^{\prime}}>+<\overline{b^{\prime} w^{\prime}}>-\epsilon_{E K E}
\end{array}
$$

where the terms on the left hand side represents the evolution of the wave kinetic energy, $E K E=\frac{1}{2}<\overline{\mathbf{u}^{\prime} \cdot \mathbf{u}^{\prime}}>$, and its transport by viscous terms, and the terms on the right hand side are the divergence of the total wave energy flux, energy exchange with the mean subinertial flow $U_{0}$, energy exchange with the mean inertial flow $\overline{\mathbf{u}}$, energy conversion to potential energy, and wave dissipation rate $\epsilon_{E K E}=\nu<\mid \overline{\left.\nabla \mathbf{u}^{\prime}\right|^{2}}>$. The total wave energy flux includes upward energy flux associated with the pressure work, downward transport of the mean subinertial and inertial flow kinetic energies, and energy transport by triple correlation term.

According to the non-acceleration theorem, there is no energy exchange between waves and the mean flow in the limit of steady and conservative waves. These assumptions are well justified right at the bottom, where waves are generated and energy radiation is well described by the linear theory, and above the breaking region, where waves become a small amplitude, and therefore, linear. Nonlinear, non-conservative processes take place in the bottom $700 \mathrm{~m}$ where waves break and dissipate most of their energy. To be consistent with the non-acceleration theory, the term representing energy conversion between the mean and the waves must be defined in terms of the divergence of the Eliassen-Palm flux which vanishes in the limit of stationary and conservative waves. It also provides an exact cancellation between the upward energy flux associated with the pressure work and the downward kinetic energy flux,

$$
<\overline{p^{\prime} w^{\prime}}>=-U_{0}\left(<\overline{w^{\prime} u^{\prime}}>-\frac{f}{N^{2}}<\overline{v^{\prime} b^{\prime}}>\right) .
$$

However, since in our problem the bulk of the energy is radiated by waves which have 
frequency greater than the inertial frequency, the correction due to the buoyancy flux $<\overline{v^{\prime} b^{\prime}}>$ is weak.

To illustrate energy pathways in the system of a mean subinertial flow, inertial oscillations and internal waves, we estimate kinetic energy budgets for the bottom $1 \mathrm{~km}$ averaged over the last several inertial periods from the $F r^{-1}=0.4$ simulation for both free-slip and no-slip bottom boundary conditions. Both mean and the wave energy budgets are closed within $5 \%$ of the energy dissipation value for this Froude number simulation. Magnitudes of the different terms of the kinetic energy equations, $\left(\mathrm{mW} \mathrm{m}^{-2}\right)$, integrated in the bottom $1 \mathrm{~km}$ are shown in the following tables for the mean,

\begin{tabular}{cccc}
\hline \hline$\partial_{t} M K E$ & $\nu \partial_{z z} M K E$ & $-<\overline{\mathbf{u}} \cdot \partial_{z} \overline{w^{\prime} \mathbf{u}^{\prime}}>$ & $-\epsilon_{M K E}$ \\
\hline $2.0(1.5)$ & $-0.1(-0.5)$ & $7.3(6.1)$ & $-5.2(-4.1)$ \\
\hline
\end{tabular}

and for the waves,

\begin{tabular}{cccc}
\hline \hline$\partial_{t} E K E$ & $\nu \partial_{z z} E K E$ & $-\partial_{z} F$ & $U_{0} \partial_{z}<\overline{w^{\prime} u^{\prime}}>$ \\
\hline$-0.2(-0.2)$ & $-0.1(-0.2)$ & $-0.1(-1.5)$ & $13.5(11.8)$ \\
\hline \hline \multicolumn{4}{c}{} \\
\hline \hline$-\partial_{z} \overline{w^{\prime} \mathbf{u}^{\prime}}>$ & $\left\langle\overline{b^{\prime} w^{\prime}}>\right.$ & $-\epsilon_{E K E}$ & \\
\hline$-7.3(-6.1)$ & $0(0.1)$ & $-5.9(-4.6)$ & \\
\hline
\end{tabular}

where $F$ is total wave kinetic energy flux, and the two values for each term are estimates from free-slip and no-slip (in parenthesis) boundary condition simulations.

From the mean kinetic energy budget we can see that inertial oscillations, and therefore, mean kinetic energy are nearly equilibrated during the last several inertial periods. Roughly $25 \%$ of the energy coming from internal waves still goes to inertial oscillation acceleration, while the rest of it is balanced by viscous dissipation. Thus, to the leading order, in the fully equilibrated state, there is a balance between energy input from the waves and energy output to dissipation by the mean,

$$
-<\overline{\mathbf{u}} \cdot \partial_{z} \overline{w^{\prime} \mathbf{u}^{\prime}}>\approx \epsilon_{M K E}
$$


Wave kinetic energy is well equilibrated during the last several periods. Energy conversion to potential energy is small. Divergence of the total wave flux is also small, resulting from a close cancellation between upward energy flux, associated with the pressure work, and downward kinetic energy fluxes. Thus, the leading order balance in the wave energy budget is,

$$
U_{0} \partial_{z}\left\langle\overline{w^{\prime} u^{\prime}}>\approx-<\overline{\mathbf{u}} \cdot \partial_{z} \overline{w^{\prime} \mathbf{u}^{\prime}}>+\epsilon_{E K E},\right.
$$

representing a balance between energy extracted by the waves from the subinertial flow $U_{0}$ and energy going to inertial oscillation and internal wave dissipation. Combining equations (2.56) and (2.57), we get,

$$
U_{0} \partial_{z}<\overline{w^{\prime} u^{\prime}}>\approx \epsilon_{M K E}+\epsilon_{E K E} .
$$

This equation is a total balance between the source and the sinks of kinetic energy in the system of a subinertial flow, inertial oscillations, and internal waves which holds quite well throughout the water column in the bottom $1 \mathrm{~km}$ (Fig. 2-11). The various energy pathways are sketched in Fig.2-12. Energy is extracted from the infinite reservoir in the prescribed subinertial flow $U_{0}$ maintained throughout the simulation. This energy is eventually dissipated as internal waves and inertial oscillations. The rate at which energy is extracted by internal waves from the mean subinertial flow is determined by the vertical divergence of the zonal component of the Eliassen-Palm flux, which is directly related to the energy radiated from topography.

Simulations with free-slip and no-slip boundary conditions are qualitatively very similar. However, there is some quantitative difference. Energy dissipation estimate in the simulation with a no-slip boundary condition reduces by about 20-30\% compared to the simulation with a free-slip condition. This reduction is likely due to a flow separation effect. In the simulation with a no-slip boundary condition, mean flow sliding down the lee side of the hill separates from topography and reduces the effective topographic height. This in turn reduces the amplitude of radiated waves and the amount of wave energy dissipation. 


\subsubsection{Wave breaking and dissipation}

The numerical simulations show that, to the leading order, the generation and dissipation of kinetic energy depends on the vertical divergence of the zonal component of the Eliassen-Palm flux. Linear theory gives a good prediction for the wave fluxes at the bottom. The vertical scale of flux divergence is determined by the wave breaking scale. In the simulations described so far the vertical scale of the wave breaking region is of the order of $700 \mathrm{~m}$ and essentially independent on $\mathrm{Fr}^{-1}$ or more specifically on the topography amplitude $h_{T}$ that we varied to change $F r^{-1}$. However, the vertical extent of the breaking region depends on the topography wavenumber $k_{T}$, and the Coriolis frequency $f$, i.e. it depends on $\chi$ and $\beta$ in (2.1) and (2.5). We find that the wave breaking scale increases with decreasing $f$, with a weaker dependence on $k_{T}$.

There are three main pathways from wave generation to wave breaking. First the radiated waves can have a sufficient amplitude to become convectively or shear unstable. This is not the case in our simulations, because the linear wave solutions are stable. Second, wave-wave interactions can transfer energy to smaller scale waves with large shears. This is the classical turbulent picture, where energy is fluxed from larger to smaller scales where instabilities and wave breaking can occur. We can rule out this pathway, because the interaction time scale estimated for wave amplitudes observed in our simulations is of the order of a day, which is long enough for waves to radiate up to 2-3 $\mathrm{km}$ before they can break. Third, modulation by background shear or changes in stratification can make the waves unstable. In our simulations a large inertial shear seems to be responsible for the observed wave breaking.

Upward propagating wave packets, which are generally stable based on the Richardson number criteria, break as they pass through the vertically sheared inertial oscillations. The effect of inertial oscillations on the propagation of internal wave packets is described by Broutman and Young (1986) based on wave ray theory. Inertial oscillations, essentially, play a role of a filter separating long internal waves which manage to pass through inertial oscillation without any substantial change, and short internal waves which are modulated by inertial oscillations causing an increase in their vertical 
wavenumbers and eventually break. Almost all wave modes generated at the bottom have a scale shorter than inertial oscillation scale and are significantly affected by the inertial oscillations. In summary the scale of the wave breaking region seen in the simulations is determined by the size of internal wave packets due to superposition of the various modes generated by the time dependent mean flow.

From the linear theory solution we can see that generated internal wave modes form wave packets as they radiate upward (Fig. 2-13). The size of these packets depends on the vertical scales of internal wave modes and the number of modes involved. Variation in the Coriolis frequency $f$, as opposed to horizontal wavenumber $k_{T}$, changes not only wave properties according to the wave dispersion relation but also an effective number of the wave modes, $f<\sigma_{n}<N$.

Finally, we diagnose turbulent dissipation rate $\epsilon$ from the simulations and compare it to the dissipation rate observed in Drake Passage. Fig. 2-14 shows vertical profiles of time averaged dissipation rate from different Froude number simulations. Values of the dissipation rate integrated over $1 \mathrm{~km}$ depth above the bottom are shown in Fig. 2-15. The magnitude of the turbulent dissipation rate generally increases with inverse Froude number as the internal wave amplitude grows and waves become more nonlinear and break. The dissipation rate is significantly intensified in the bottom 1 $\mathrm{km}$ where most of the wave breaking takes place, and then decays in ocean interior. The dissipation rate integrated over the bottom $1 \mathrm{~km}$ saturates at about $25 \mathrm{~mW}$

$\mathrm{m}^{-2}$ for inverse Froude number higher that 0.7 . The dissipation rate estimated for inverse Froude number 0.6, characteristic of Drake Passage region, is about $20 \mathrm{~mW}$ $\mathrm{m}^{-2}$. This number compares well with the estimate of the dissipation rate in Drake Passage obtained from observations (Naveira-Garabato et al., 2004).

\subsection{Conclusions}

Estimates of turbulent mixing inferred from high wavenumber fluctuations in density and velocity profiles suggest that turbulent kinetic energy dissipation is strongly enhanced in a few sectors of the ACC. Diapycnal mixing is concentrated in the bottom 
kilometer where topography is rough. In this chapter we showed through theory and numerical simulations that the observed dissipation can be sustained by breaking internal waves generated by geostrophic eddies impinging on topographic hills a few kilometers wide. This is in contrast to other regions of the ocean where tidal flows seem to be the primary generator of internal waves. The difference arise because bottom geostrophic eddies are anomalously large in the Southern Ocean, while tides are weak.

This is an idealized study, but we have chosen parameters that mimic geostrophic flows and topographic features found in the Drake Passage sector of the ACC, one of the very few places where dissipation estimates from observations are available. In this region geostrophic eddies radiate internal waves from a limited range of topographic horizontal scales, varying from about $600 \mathrm{~m}$ to $6 \mathrm{~km}$, with most of the radiation due to topographic features of 1-3 km scales. The vertical scale of the radiated internal waves is about $600 \mathrm{~m}$, significantly shorter than the local ocean depth. The inverse Froude number $\mathrm{Fr}^{-1}$ is about 0.6, i.e. topographic slopes are sub-critical. We find that energy radiation in this regime is characterized by a time-dependent, multichromatic waves.

A novel result of this chapter is that upward radiating time-dependent internal waves trigger inertial oscillations at the ocean bottom. In turn the inertial oscillations modify the wave generation process and result in radiation of time dependent, multichromatic internal waves. The feedback between the waves and the inertial flow leads to a resonance which reinforces inertial oscillations. The inertial oscillations grow to become as large as the geostrophic flow and have a substantial amount of vertical shear on scales of a few hundred meters. The inertial shear strongly modulates the upward propagating packets of short internal waves and promotes enhanced wave breaking. We explored the resonant feedback mechanism within a weakly nonlinear framework and demonstrated that the analytically predicted growth rate and properties of inertial oscillations are consistent with the results of numerical simulations. Numerical simulations show that a substantial fraction, 30\%, of radiated energy dissipates locally in the bottom kilometer, as waves are tilted by the inertial shear, and 
break.

There is another instability mechanism, the Parametric Subharmonic Instability (PSI), which is characterized by energy transfer to near-inertial frequency. This instability results from a resonant triad interaction where the primary wave, recharged, for instance, by the barotropic tide interacting with the bottom topography, transfers energy to the two other component of the triad at half the frequency. The PSI mechanism is very efficient at transferring energy to near-inertial frequency at latitudes where the tidal frequency is twice the inertial frequency. The resonant feedback mechanism described in this study is different from the PSI. The superposition of a geostrophic flow and an inertial oscillation over the bottom topography generates a pair of waves: one is a lee wave, forced by the geostrophic flow, and the other is a harmonic of the inertial frequency, forced by the inertial oscillation. These two waves transfer energy to $f$ through a triad interaction, different from the PSI. In particular the triad would not be very efficient in the absence of the bottom boundary condition, unlike the PSI (McComas and Bretherton, 1977). The boundary conditions are key because, as these waves break and deposit their momentum, they re-energize inertial oscillation in the zonally averaged flow. A stronger inertial component of the zonally averaged flow energizes the initial pair of waves and the feedback continues. As a results, inertial oscillation grows at the expense of the mean flow. In a sense this is a problem of wave-mean flow interaction, while the PSI is a wave-wave interaction. Energy is put in the two carrier waves with the boundary condition and, then, effectively transferred to the inertial frequency, $f$, as the carrier waves break.

The numerical simulation setup (2-D and zonally periodic domain) is such that the inertial oscillations cannot radiate horizontally and, therefore, always stay in the region of strong geostrophic flow above rough topography where they get reinforced by internal waves through the resonant feedback. In the ocean, however, these oscillations are modulated by the large-scale variations in the mean flow. This modulation imposes a horizontal scale on oscillations making them near-inertial and allowing them to radiate both horizontally and vertically. Using a geostrophic eddy scale of $100 \mathrm{~km}$ as the horizontal scale of near-inertial oscillations and $1 \mathrm{~km}$ as their verti- 
cal scale, we find, estimating wave group velocities, that it takes near-inertial waves roughly 20 days to radiate an eddy scale away. This time scale is an order of magnitude longer than the characteristic growth rate, roughly 2 days, associated with the resonant feedback mechanism. Over the 2-day time period near-inertial oscillations radiate horizontally a distance of only about $5 \mathrm{~km}$. Changes in the Coriolis frequency on this distance due to the beta effect and geostrophic flow shear are insignificant, and are of the order of $0.1 \%$ of $f$. Hence we expect the feedback to occur also in a fully developed geostrophic eddy field.

In this chapter we considered one-dimensional monochromatic topography. In Chapter 3 we show that the results are very similar for multichromatic topography, because radiation is dominated by a few topographic scales. In Chapter 4 we further extend the analysis to 3-D, monochromatic simulations and shows that wave radiation is similar to the 2-D experiments.

Future work will need to address the degree to which radiation and breaking is sensitive to the choice of bottom boundary condition. In this chapter we considered free-slip and no-slip conditions and found that in the latter case wave dissipation was reduced by about 20-30\%; the problems were otherwise very similar. We are led to believe that a more appropriate boundary condition capable of resolving the stress in the logarithmic layer will bring quantitative, but no qualitative corrections. But the question deserves more study.

The partial absence of meridional boundaries makes the Southern Ocean a special place where the geostrophic field has to equilibrate through dissipation at the bottom boundary. QG turbulence theory suggests that this is achieved through an inverse cascade where the geostrophic eddies develop a large barotropic component. In this study we show that eddies can equilibrate by generating internal waves from the bottom topography which radiate into the ocean interior and break, mostly within the bottom kilometer. This result might therefore be germane to the Southern Ocean. In mid-latitudes barotropization is arrested by lateral boundaries and the bottom velocities do not grow large enough to overcome the barotropic tidal signal. 


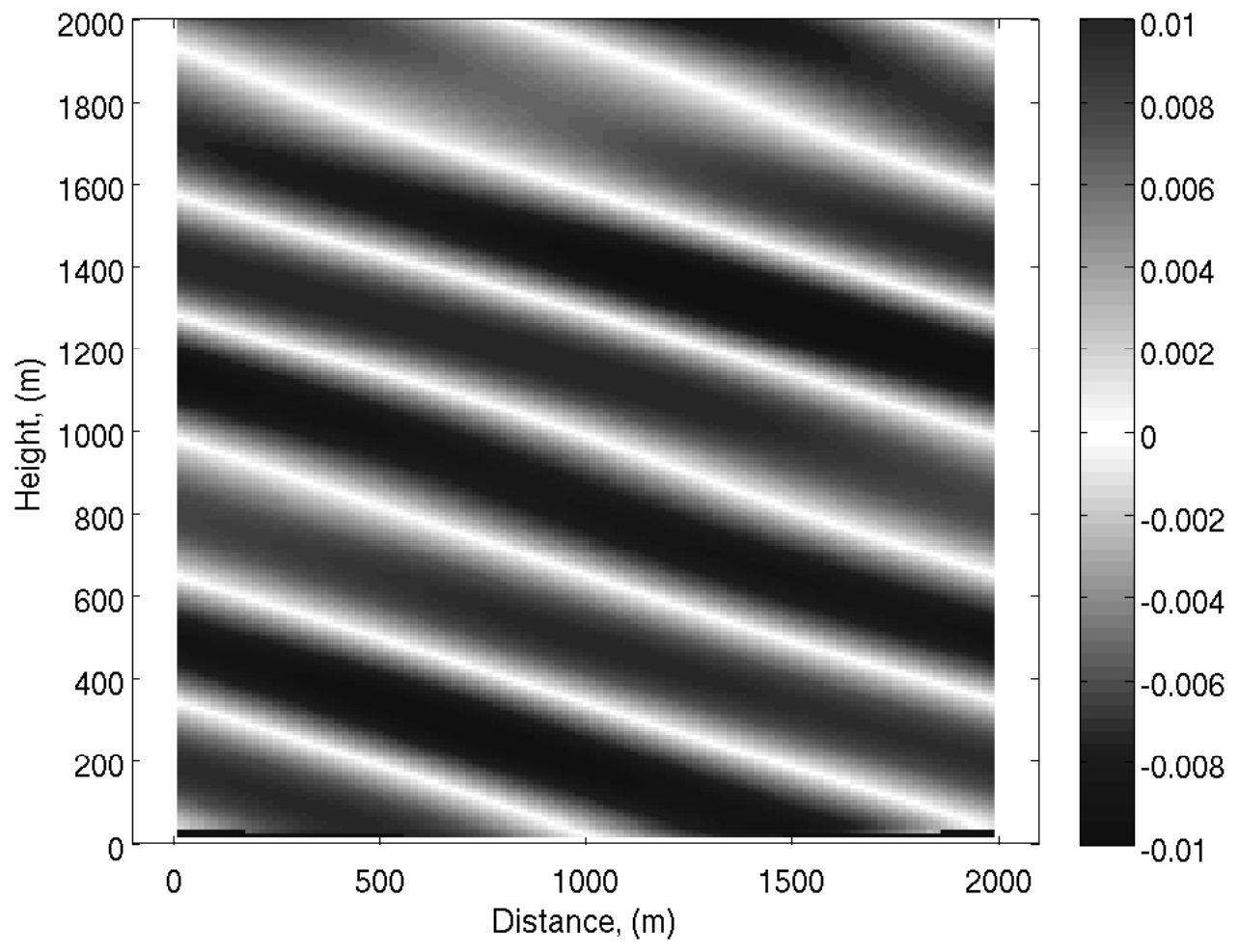

Figure 2-1: Snapshot of the wave zonal velocity $\left(\mathrm{m} \mathrm{s}^{-1}\right)$ from $F r^{-1}=0.1$ simulation. 


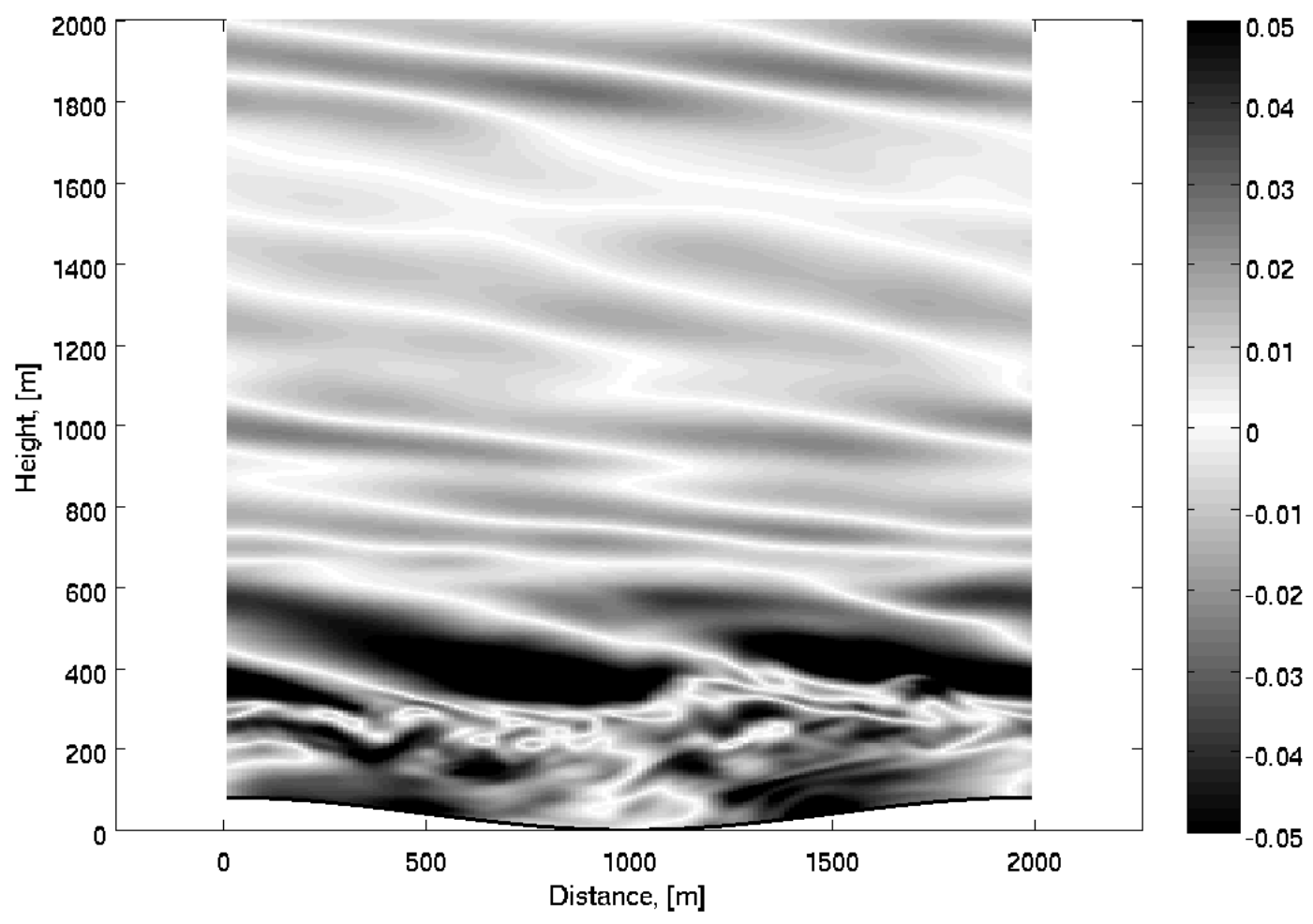

Figure 2-2: Snapshot of the wave zonal velocity $\left(\mathrm{m} \mathrm{s}^{-1}\right)$ from $F r^{-1}=0.4$ simulation. 

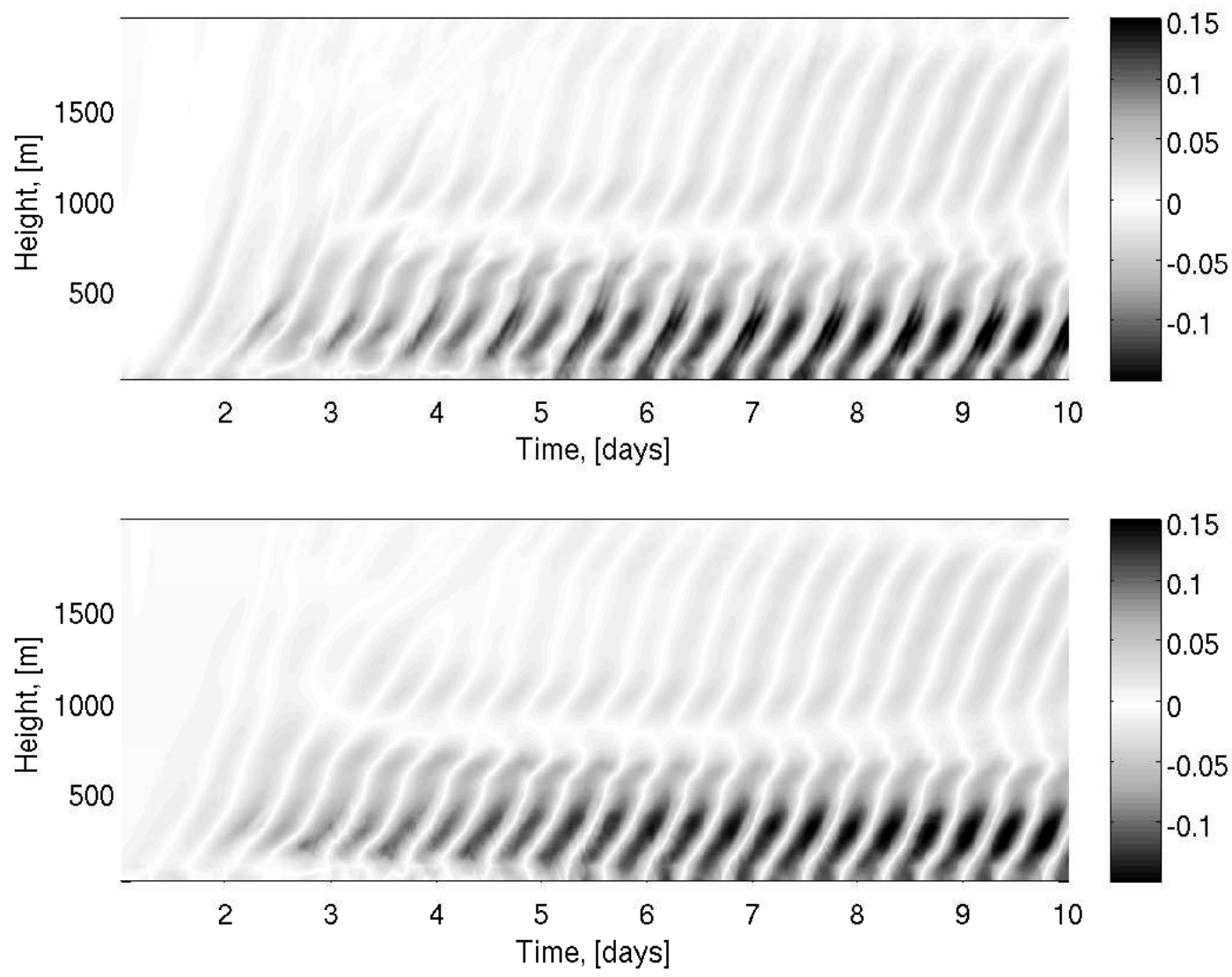

Figure 2-3: Time evolution of zonally averaged velocities $\left(\mathrm{m} \mathrm{s}^{-1}\right)$ from $F r^{-1}=0.4$ simulation: (upper panel) deviation from an externally prescribed $0.1 \mathrm{~m} \mathrm{~s}^{-1}$ zonal mean flow,(lower panel) meridional velocity component. 


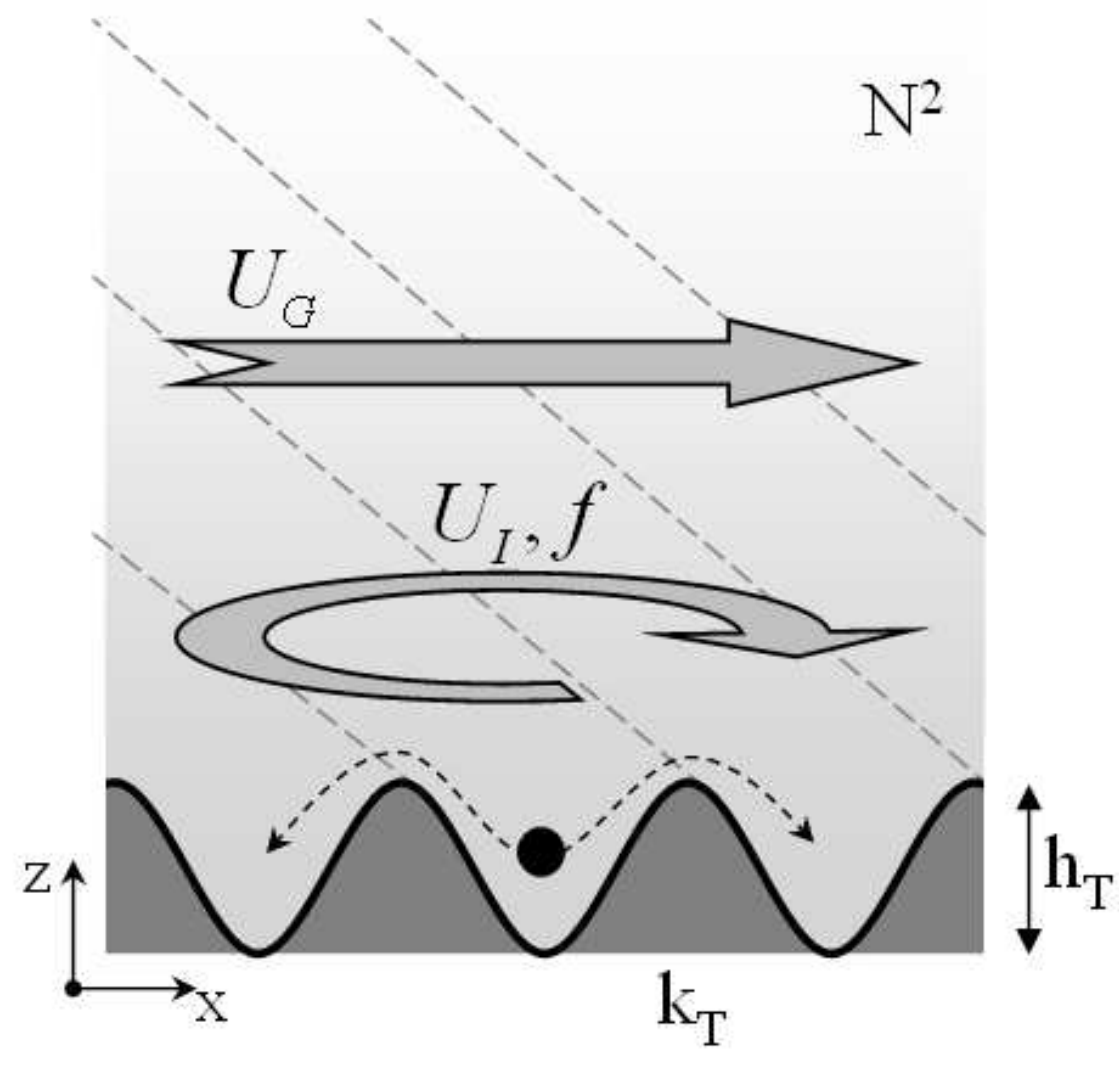

Figure 2-4: Definition sketch illustrating interaction of a geostrophic flow $U_{G}$ and an inertial oscillation $\left(U_{I}, f\right)$ with periodic bottom topography $\left(k_{T}, h_{T}\right)$ in stably stratified fluid. Dashed gray lines are the phase lines of the waves and the dashed black line is the trajectory of a particle, illustrating its excursion during one period of oscillation. 


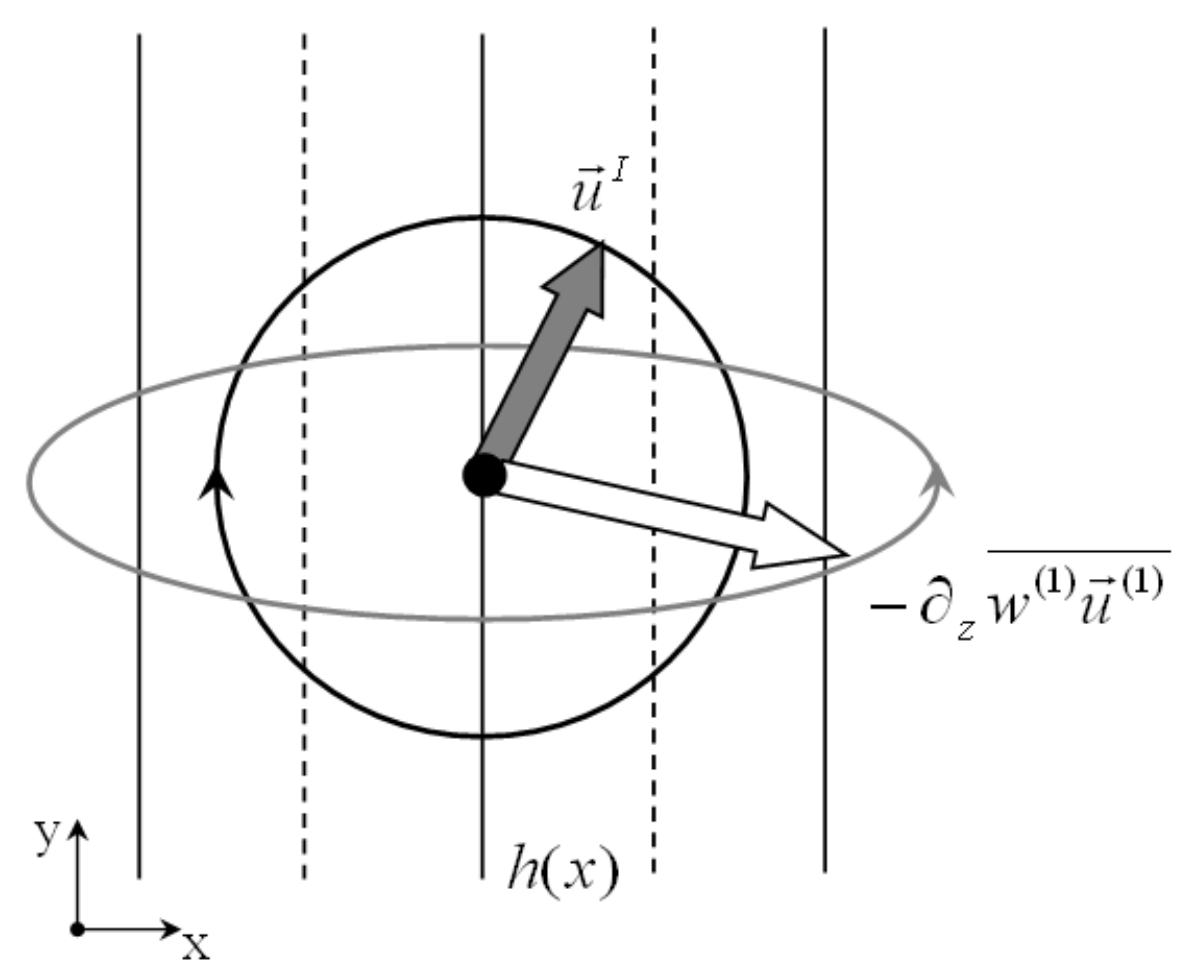

Figure 2-5: Sketch illustrating resonant forcing mechanism driving inertial oscillations. Straight and dashed vertical lines are the topography crests and troughs, respectively. Gray and white arrows show, respectively, the direction of inertial oscillation velocity vector and the direction of the wave momentum flux divergence. Circular (black) and elliptical (gray) trajectories indicate the direction of rotation for the inertial oscillation $(f>0)$ and flux divergence vectors, respectively. 


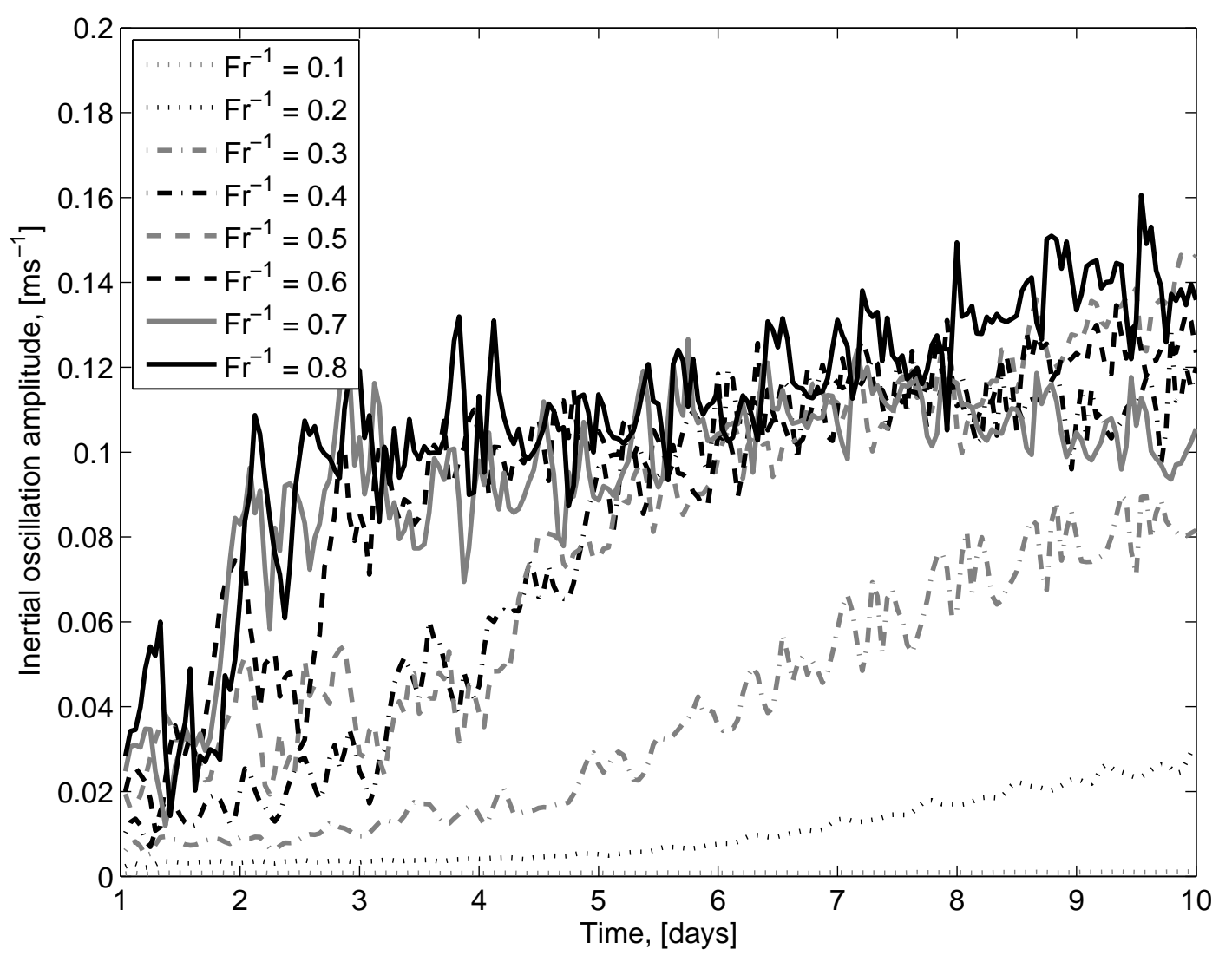

Figure 2-6: Evolution of the bottom value of the inertial oscillation amplitude (m $\mathrm{s}^{-1}$ ) from different simulations. 


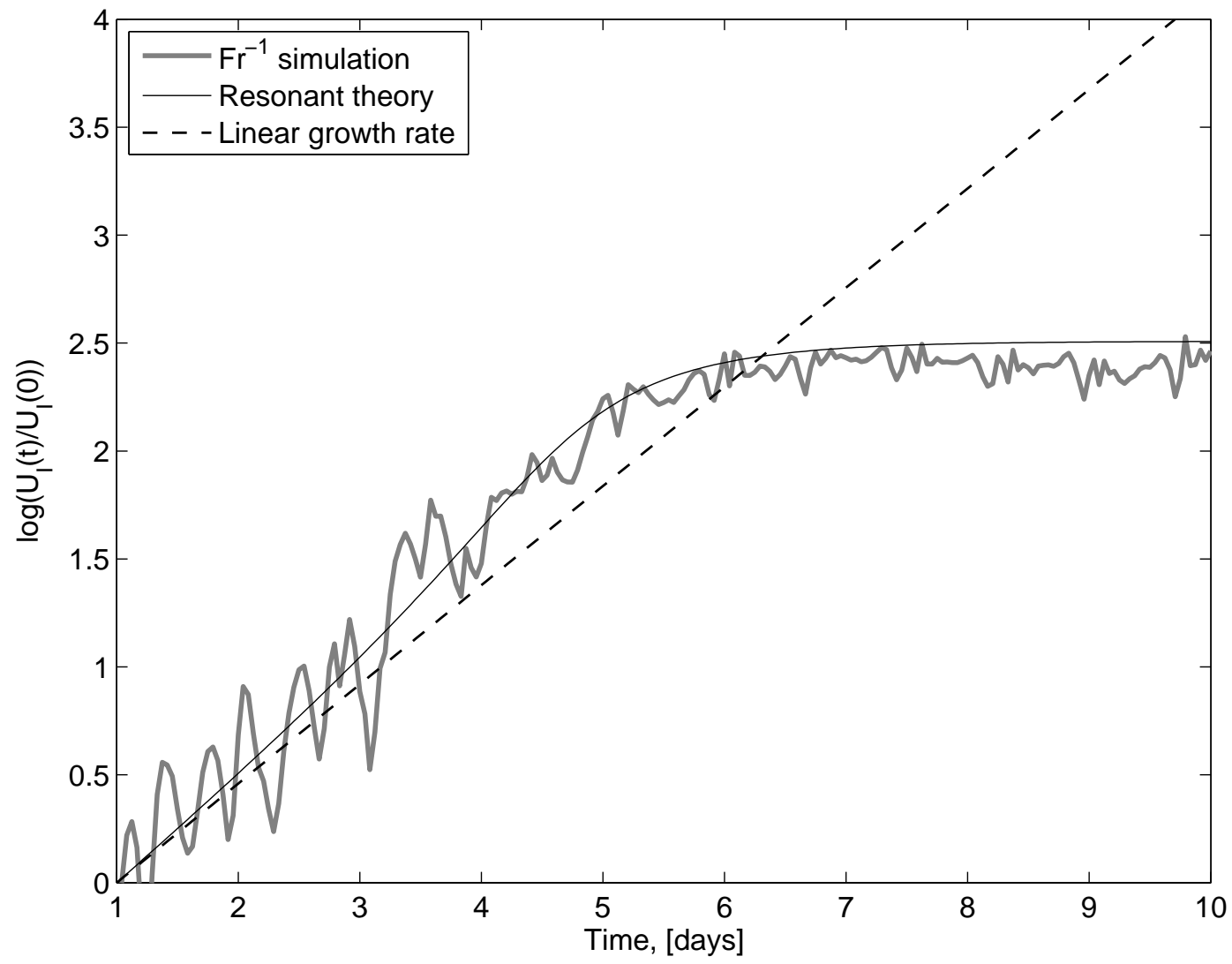

Figure 2-7: Evolution of the bottom value of the inertial oscillation amplitude normalized by its initial value, $\log \frac{U_{I}(t)}{U_{I}(0)}$, from the $\mathrm{Fr}^{-1}=0.4$ simulation (gray), resonant feedback theory prediction from (2.36) and (2.38) (solid black), and linear growth rate prediction (2.49) (dashed black). 




Figure 2-8: Profiles of the vertical energy flux $\left(\mathrm{mW} \mathrm{m}^{-2}\right)$ from different simulations. 


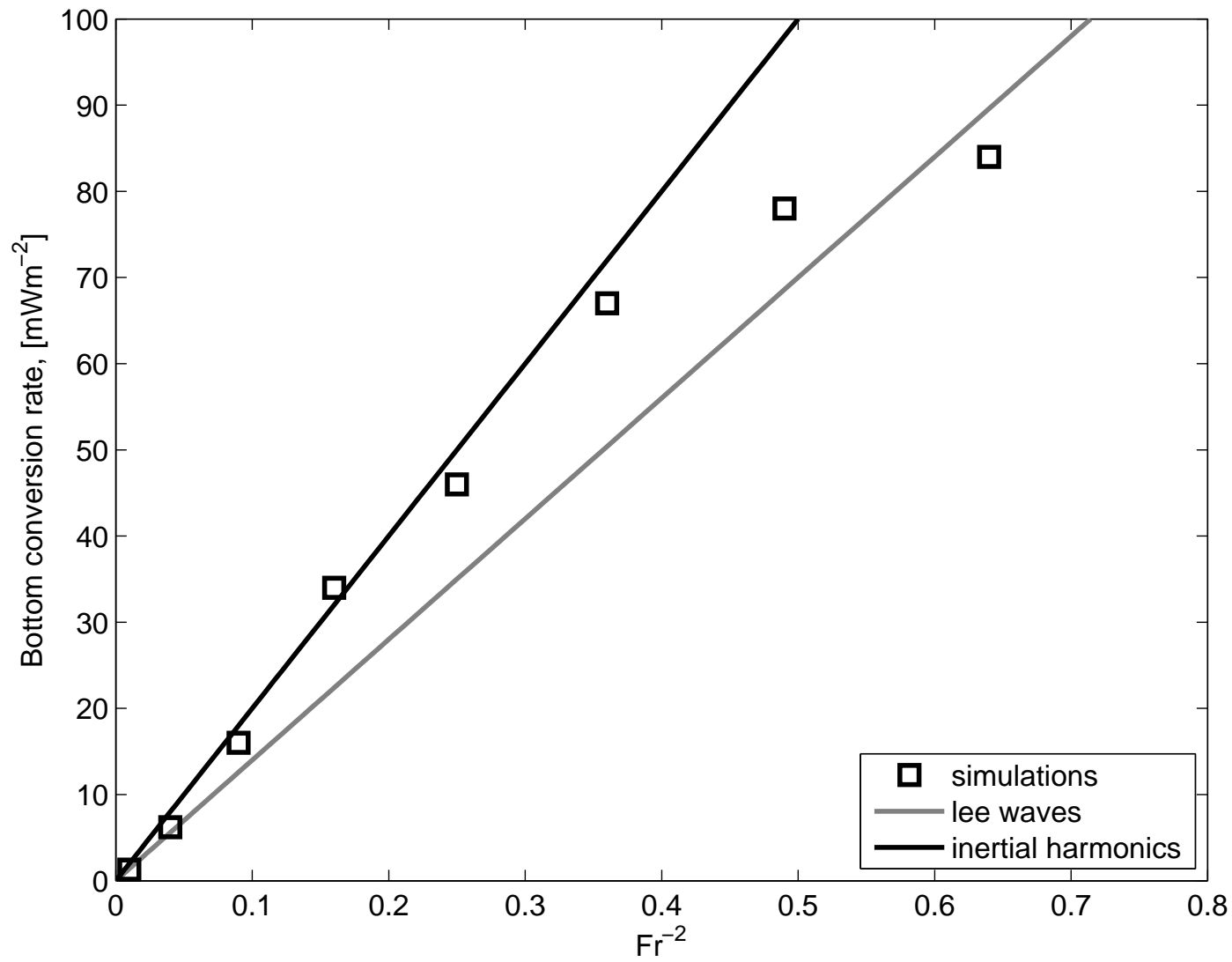

Figure 2-9: Energy conversion rate at the bottom as a function of $\mathrm{Fr}^{-2}$ : diagnosed from simulations (black squares), predicted from linear lee wave theory (gray line), and predicted from linear theory for inertial harmonics (black line). 


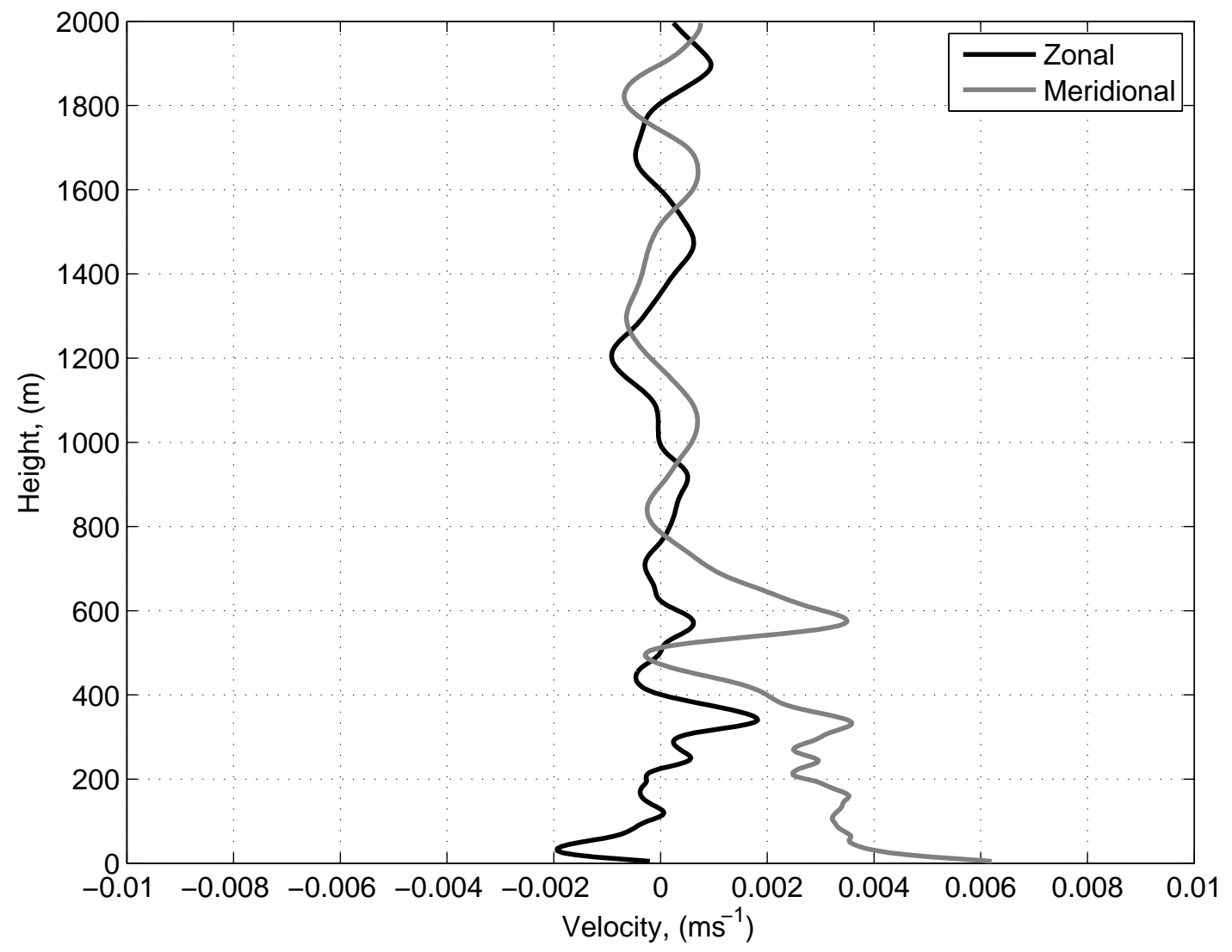

Figure 2-10: Zonal and meridional velocity components from $F r^{-1}=0.4$ simulation averaged over several inertial periods. 


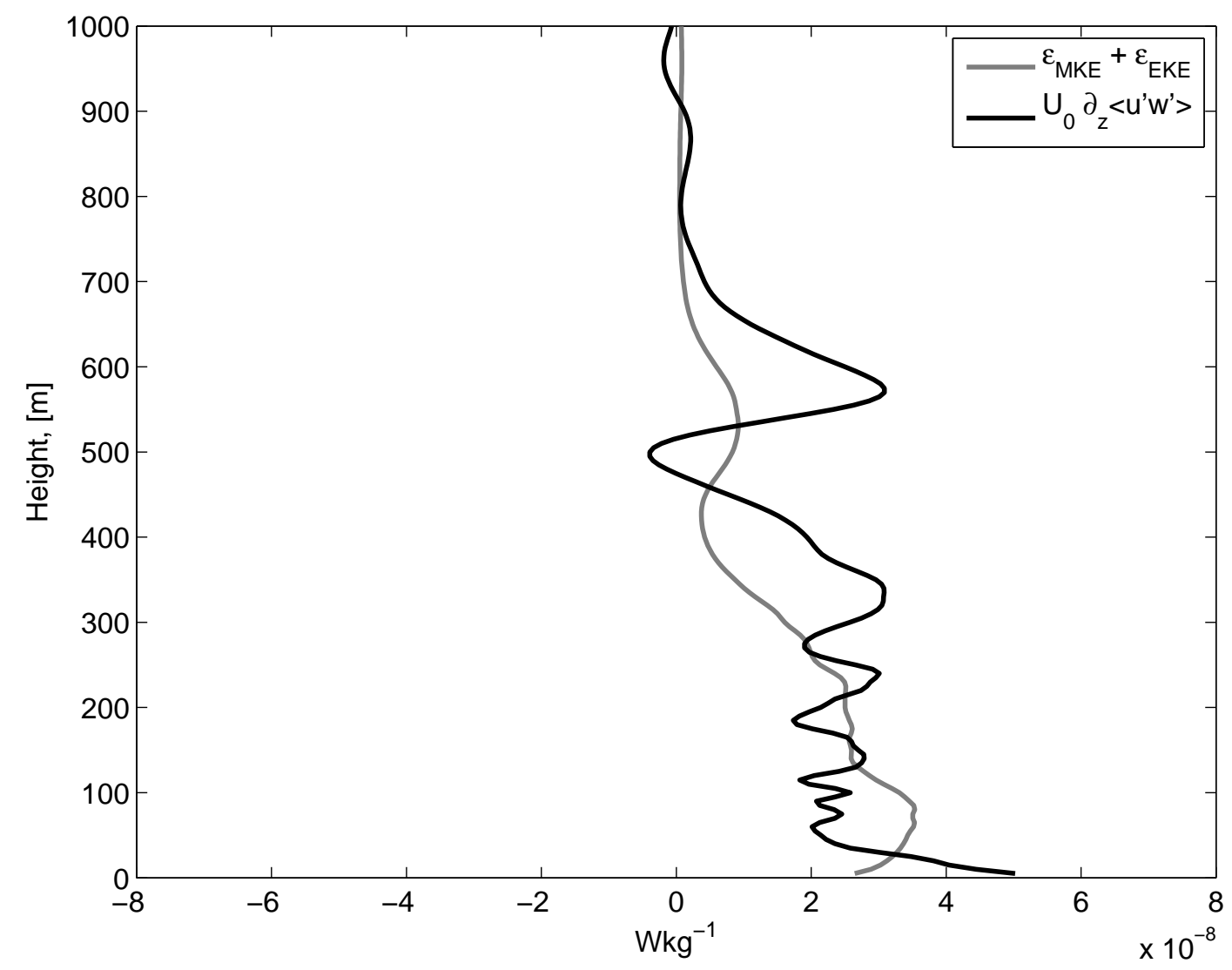

Figure 2-11: Vertical profiles of the total energy dissipation rate (gray) and energy conversion from the mean subinertial flow into internal waves (black) $\left(\mathrm{W} \mathrm{kg}^{-1}\right)$ for $F r^{-1}=0.4$ simulation. 


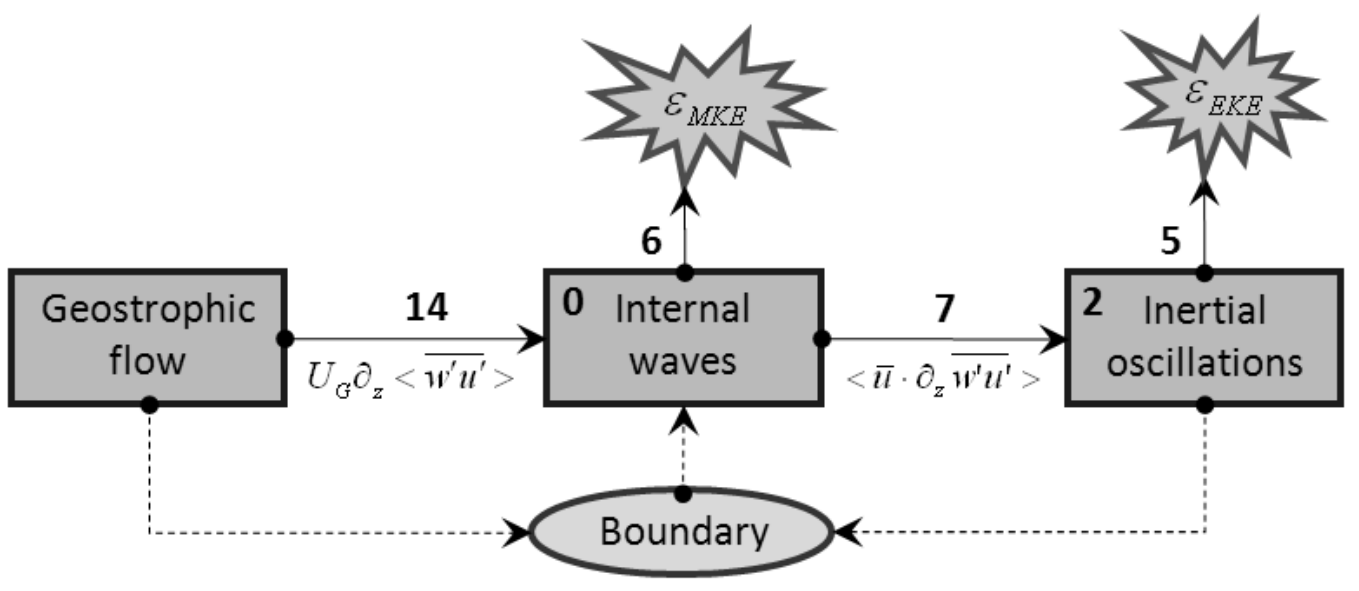

Figure 2-12: Diagram of energy pathways. Energy conversion and dissipation values $\left(\mathrm{mW} \mathrm{m}^{-2}\right)$ are from $\mathrm{Fr}^{-1}=0.4$ simulation. Values inside boxes represent growth (time derivative) of kinetic energy. 


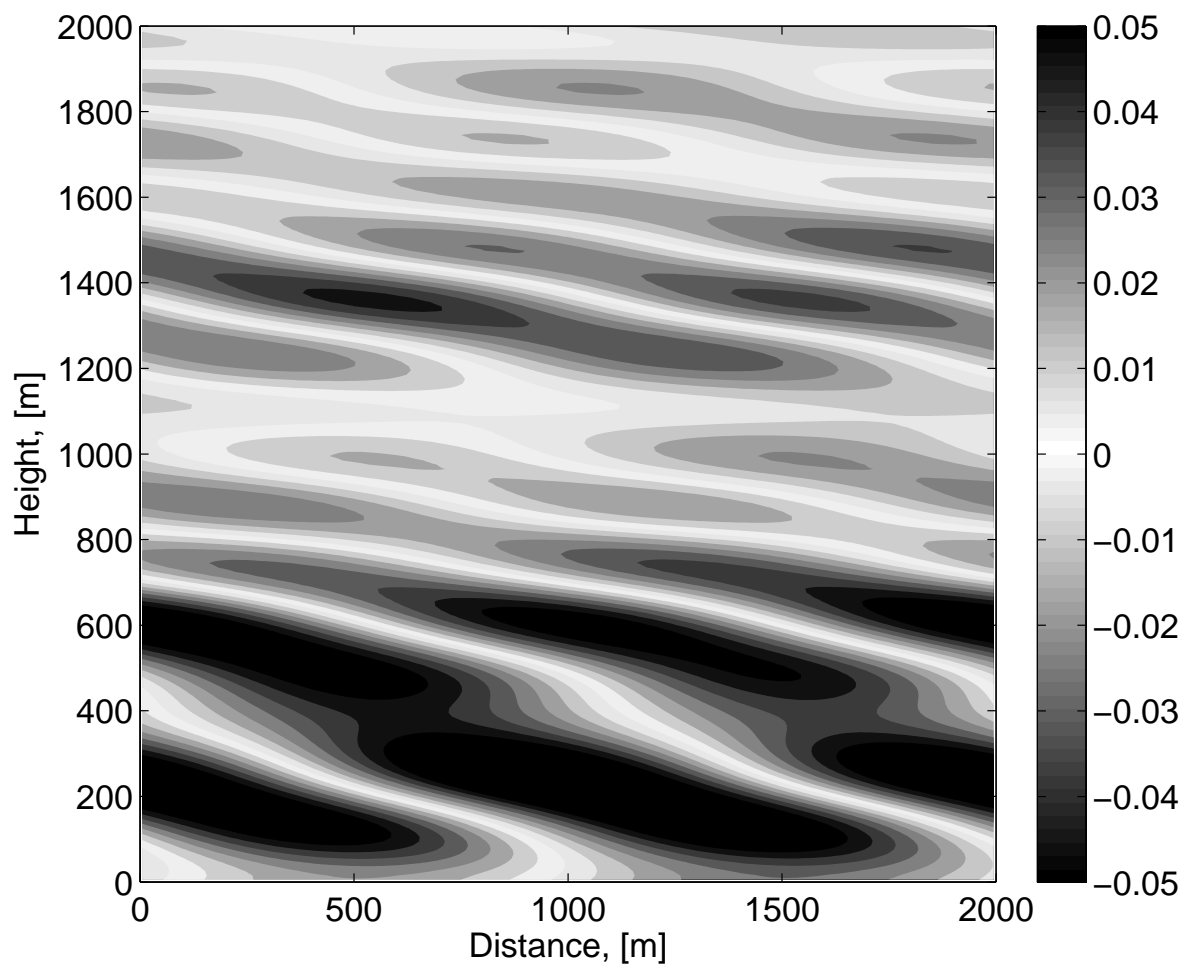

Figure 2-13: Wave zonal velocity $\left(\mathrm{m} \mathrm{s}^{-1}\right)$ from the linear solution in (2.32) corresponding to $\mathrm{Fr}^{-1}=0.4$ simulation. 


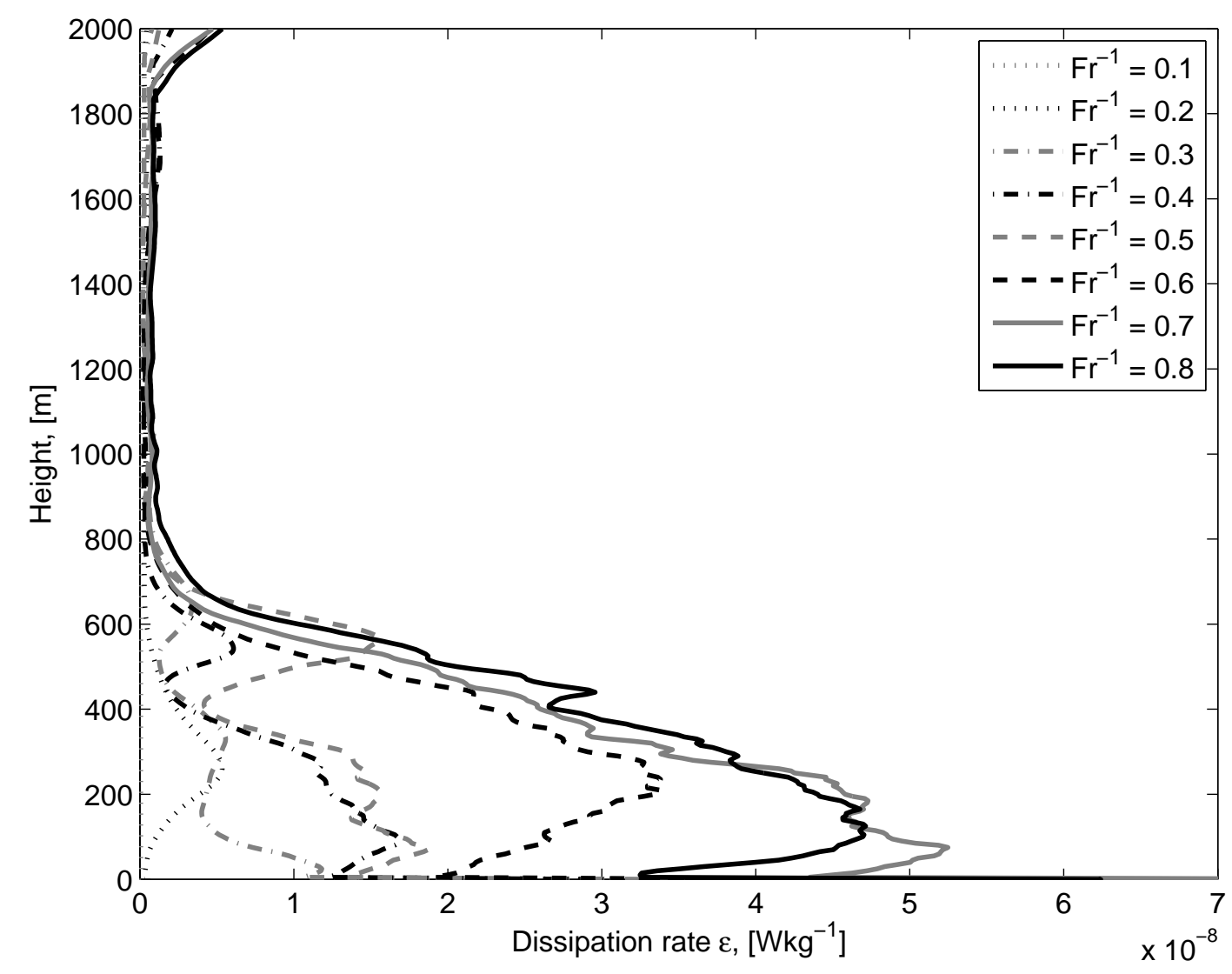

Figure 2-14: Vertical profiles of energy dissipation rate $\left(\mathrm{W} \mathrm{kg}^{-1}\right)$ from different simulations. 


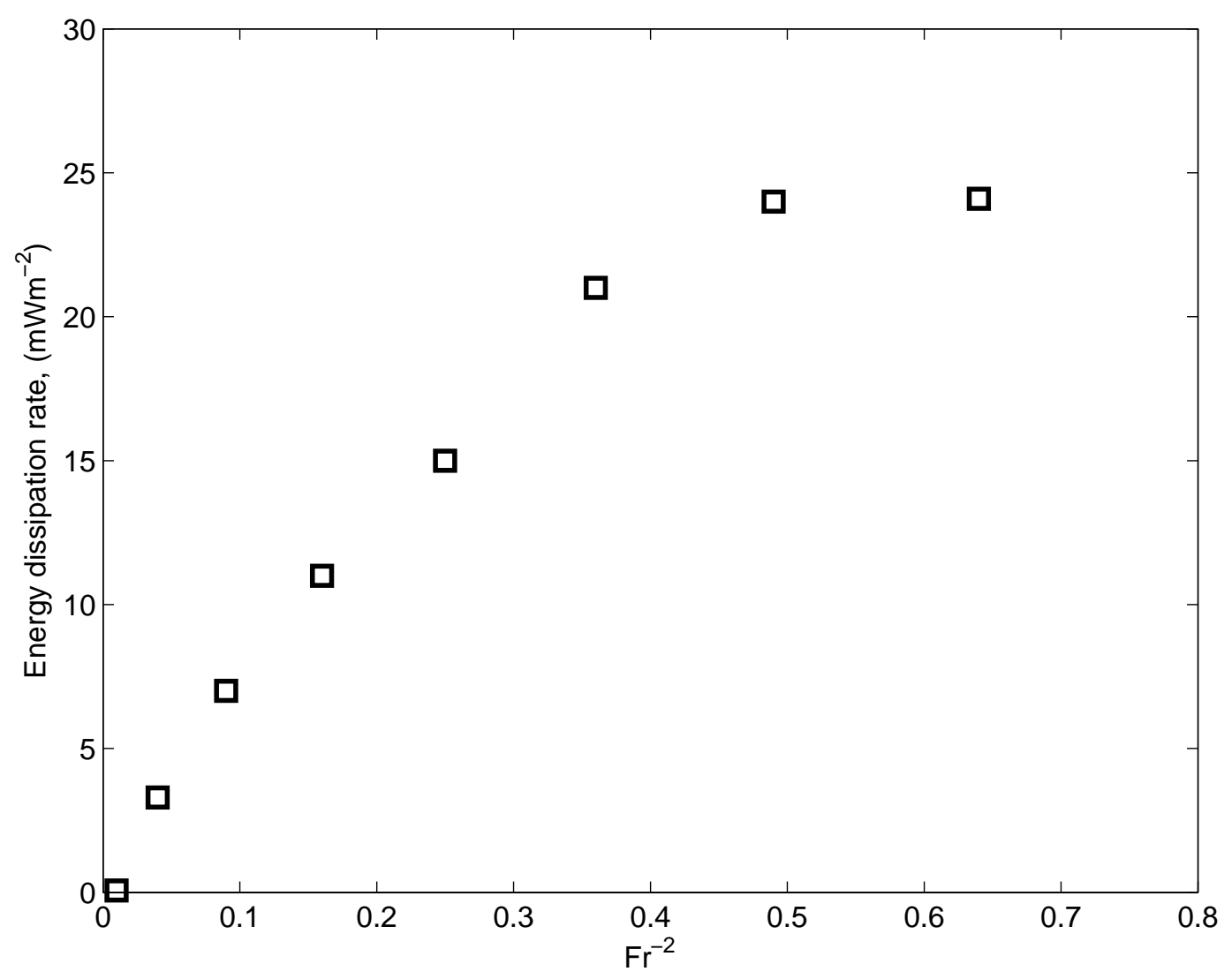

Figure 2-15: Energy dissipation rate $\left(\mathrm{mW} \mathrm{m}^{-2}\right)$ integrated in bottom $1 \mathrm{~km}$. 


\section{Chapter 3}

\section{Application to the Southern Ocean}

\subsection{Introduction}

Ocean mixing sets the stratification of much of the global ocean by upwelling of dense, deep waters formed in polar regions (Wunsch and Ferrari, 2004). Mixing is especially important in the Southern Ocean where the Meridional Overturning Circulation (MOC) of the global ocean is largely powered. However little is known about what dynamics supports that mixing.

The Southern Ocean part of the MOC consists of an upper and a lower cell (Speer et al., 2000). Theories suggest that the upper cell is driven by atmospheric forcing at the surface with an adiabatic return flow at depth (Rintoul et al., 2001; Marshall and Radko, 2003; Olbers et al., 2004). Much less is known about the dynamics of the lower cell, except that diabatic mixing is essential to explain the latitudinal change in deep water mass properties (Ito and Marshall, 2008). Inverse analyses of the Southern Ocean hydrography imply that high mixing rates in the deep Southern Ocean are required to close the heat and salt budgets (Ganachaud and Wunsch, 2000; Sloyan and Rintoul, 2001). Energy dissipation estimates from observations confirm widespread high mixing rates above the bottom boundary layer associated with radiation of internal waves from topography (Naveira-Garabato et al., 2004). Enhanced abyssal mixing has been linked to the radiation of internal tides in other parts of the ocean (Polzin et al., 1997; Ledwell et al., 2000; St.-Laurent and Garrett, 2002), 
however, it does not seem to be the case in the Southern Ocean where abyssal flows are dominated by geostrophic eddies (Naveira-Garabato et al., 2003). In Chapter 2 we showed that geostrophic motions are very efficient at radiating waves from small topographic features, resulting in local mixing. In the Southern Ocean geostrophic eddy flows penetrate all the way to the bottom. Here we test the hypothesis that the observed abyssal mixing can be explained by radiation and breaking of waves resulting from geostrophic flows impinging on small-scale topography.

Wunsch (1998) demonstrated that about $80 \%$ of the wind work on the general circulation of the global ocean is done in the Southern Ocean, and conjectured that the Southern Ocean can host intense diapycnal mixing if some fraction of that work were dissipated locally. Estimates of turbulent mixing inferred from high wavenumber fluctuations in density and velocity profiles confirm that in the Southern Ocean turbulent kinetic energy dissipation and diapycnal mixing are enhanced by orders of magnitude above background values found in most of the ocean (Naveira-Garabato et al., 2004). The observed diapycnal mixing is concentrated in the bottom $1 \mathrm{~km}$ and in regions of rough topography. For example, the vertically integrated dissipation rate averaged for a few sections across Drake Passage is reported to be of the order of $10 \mathrm{~mW} \mathrm{~m}^{-2}$, corresponding to a bottom diapycnal diffusivity of $10^{-2} \mathrm{~m}^{2} \mathrm{~s}^{-1}$. These values are an order of magnitude larger than those from the southeast Pacific region of the Southern Ocean where bottom topography is smooth.

Moored observations in Drake Passage show that the bulk of the kinetic energy is partitioned between geostrophic subinertial flows, inertial oscillations, and tides, in addition to the internal wave continuum (Nowlin et al., 1986). Any one of these motions can generate internal waves through interaction with bottom topography. However, theories of topographic wave generation have focused on the barotropic tidal component, which is believed to dominate wave radiation in mid latitudes (Bell, 1975a; Khatiwala, 2003; Llewellyn-Smith and Young, 2002). Wave generation by the dominant $\mathrm{M}_{2}$ tide component is estimated globally by Nycander (2005) using the linear theory developed by Llewellyn-Smith and Young (2002), tidal velocities from the global tidal model of Egbert and Erofeeva (2002), and bottom topography 
from satellite altimetry (Smith and Sandwell, 1997). The tidal energy flux into ocean interior from topography deeper than $2 \mathrm{~km}$ for Drake Passage is estimated to be about 1-2 $\mathrm{mW} \mathrm{m}^{-2}$. Thus, the amount of tidal energy available to support local dissipation in the Drake Passage region is an order of magnitude smaller than the observed energy dissipation rate. In Chapter 2 we showed that geostrophic flows impinging upon bottom topography are very effective in driving turbulence and mixing locally. Using idealized numerical simulations with parameters characteristic of Drake Passage, we predict energy dissipation rates up to $20 \mathrm{~mW} \mathrm{~m}^{-2}$.

In this chapter we extend the theoretical framework developed in Chapter 2 to estimate internal wave generation by geostrophic flows in two regions of the Southern Ocean: Drake Passage and the Southeast Pacific. In section 2, we describe available velocity, stratification and topography data. An analytical representation of the topography spectrum is also presented and discussed. In section 3, we discuss linear theory of wave radiation by geostrophic flows for arbitrary topography. In section 4 , we present the energy conversion estimates. In section 5, we test the theoretical estimates versus numerical simulations and observations. Finally, results are summarized in section 6 .

\subsection{Data}

The characteristics of waves radiated by a geostrophic flow impinging on topography depends on the spectrum of short topographic hills as well as bottom values of geostrophic velocity and stratification. Mean velocities and topography data are available for two different regions of the Southern Ocean: Drake Passage and the Southeast Pacific. These two regions are characterized, respectively, by high and low rates of abyssal mixing. A major goal of this chapter is to test whether wave radiation theory can reproduce the observed magnitudes and spatial variations of abyssal mixing. 


\subsubsection{Mean flow characteristics}

Bottom velocity and stratification are estimated with CTD and LADCP data from sections across western Drake Passage and in the Southeast Pacific (Fig. 1-2). Velocity and hydrographic sections across Drake Passage were collected as a part of the "Antarctic Large-Scale Box Analysis and the Role of the Scotia Sea" (ALBATROSS) cruise in March 1999. Details of the data collection and analysis methods are given in the cruise report (Heywood and Stevens, 2000), and a complete description of the CTD and LADCP observations is in Naveira-Garabato et al. (2002) and NaveiraGarabato et al. (2003). Velocity and hydrography data used for the Southeast Pacific region were collected during the World Ocean Circulation Experiment (WOCE) along the P18 line (Kunze et al., 2006).

Wunsch (1997) demonstrated that the bulk of geostrophic velocity is in low vertical modes (barotropic and baroclinic mode one), while internal waves dominate at high modes. A. Naveira Garabato (personal communication) suggests that the barotropic tidal signal is weak in the data, and the comparison between geostrophic and averaged LADCP shear implies that the geostrophic signal dominates at scales greater than 100-200 m while smaller scales are dominated by waves. To filter out high-mode internal waves, bottom velocity and stratification are defined as an average over the bottom $500 \mathrm{~m}$. In addition, there is an uncertainty in the direction of the mean flow, due to errors in both the LADCP compass heading ( $\sim 5$ degrees) and the magnitude of the velocity components $\left(\sim 1 \mathrm{~cm} \mathrm{~s}^{-1}\right)$. However, this is not an issue for the calculation presented below because, due to lack of information of flow-topography orientation, we consider all possible flow orientations rather than a particular flow direction found in the data.

The Drake Passage stratification decays with depth with significant variation in the thermocline and little in the abyssal ocean Fig. 3-1. Bottom values of buoyancy frequency range from $10^{-3} \mathrm{~s}^{-1}$ north of Drake Passage to $0.5 \times 10^{-3} \mathrm{~s}^{-1}$ in the south (Fig. 3-5), with a value of about $10^{-3} \mathrm{~s}^{-1}$ in the Polar ACC front, the region where bottom velocity and wave radiation are largest. 
The velocity field (Fig. 3-2) is dominated by the ACC fronts, peaking at the surface of the ocean, decaying in the upper $1 \mathrm{~km}$ (the thermocline) and remaining essentially constant below. Surface velocities up to $50 \mathrm{~cm} \mathrm{~s}^{-1}$ are associated with the Sub Antarctic Front, the Polar Front and the South ACC Front. The corresponding bottom velocities reach up to $10-20 \mathrm{~cm} \mathrm{~s}^{-1}$. Outside the fronts, bottom velocities are smaller $\simeq 1-2 \mathrm{~cm} \mathrm{~s}^{-1}$ (Fig. 3-6).

Data in the Southeast Pacific (Fig. 3-3 and 3-4) have a non-uniform spatial resolution with about $12 \mathrm{~km}$ spacing within station clusters separated by gaps up to 50 $\mathrm{km}$ wide. Bottom values of velocity and stratification in the $67^{\circ} \mathrm{S}-55^{\circ} \mathrm{S}$ latitudinal range are close to those in Drake Passage (Fig. 3-5 and 3-6). The stratification is slightly lower and varies from $0.5 \times 10^{-3} \mathrm{~s}^{-1}$ in the south to about $0.7 \times 10^{-3} \mathrm{~s}^{-1}$ in the north. The bottom velocity decreases equatorward from about $15 \mathrm{~cm} \mathrm{~s}^{-1}$ to $5 \mathrm{~cm}$ $\mathrm{s}^{-1}$ without a clear signature of the ACC fronts.

\subsubsection{Topography characteristics}

According to linear theory, a mean subinertial flow with bottom velocity of $U_{0}$ and bottom stratification of $N$ can generate radiating internal waves from topographic scales $k$ in the range,

$$
\frac{f}{U_{0}}<k<\frac{N}{U_{0}}
$$

which spans wavelengths from about $600 \mathrm{~m}$ to $6 \mathrm{~km}$ for $U_{0}=10 \mathrm{~cm} \mathrm{~s}^{-1}$ and $N=$ $10^{-3} \mathrm{~s}^{-1}$, characteristic values of the Southern Ocean. Topographic features with scales shorter than 15-20 km are not well resolved by satellite bathymetry (Smith and Sandwell, 1997) and, are only available in regions where high resolution multibeam topography data were collected. However, Goff and Jordan (1988) showed that the statistical properties of topography at smaller scales can be inferred from those at larger scales with a simple spectral model relating 10-100 km topographic features to smaller abyssal hills. We use this model to infer the characteristics of topography on the range of radiative scales given by (3.1).

Multibeam topography data in the Drake Passage region has been collected by 
the British Antarctic Survey. These data superimposed on the bathymetry data from satellite altimetry (Smith and Sandwell, 1997) are shown in Fig. 3-7. Drake Passage is characterized by relatively rough bottom topography compared to the upstream abyssal plains of the Southeast Pacific. Bottom topography is dominated by ridges at scales larger than roughly $100 \mathrm{~km}$. Abyssal hills at smaller scales are ubiquitous in the multi-beam topography, but they are not visible in the satellite-based data.

We compute a characteristic two-dimensional topography spectrum as an average over spectra from several different regions up to $100 \mathrm{~km}$ x $100 \mathrm{~km}$ wide which are well covered by the multibeam topography data. The spectrum is normalized such that the integrated spectrum gives the topography mean square height,

$$
\overline{h^{2}}=\frac{1}{4 \pi^{2}} \int_{-\infty}^{+\infty} \int_{-\infty}^{+\infty} \mathcal{P}(\mathbf{k}) d \mathbf{k}
$$

where $\mathcal{P}(\mathbf{k})$ is topography spectrum, and $\mathbf{k}=(k, l)$ is the horizontal wave vector. The two-dimensional spectrum of Drake Passage topography is shown in Fig. 38 as a function of zonal and meridional wavenumbers. The spectrum is described well by the model proposed by Goff and Jordan (1988), which is based on statistical description of abyssal hills morphology. Small-scale abyssal hills are formed by ridgecrest processes, off-ridge tectonics and vulcanism at larger scales. They are anisotropic in general and tend to be elongated perpendicular to the direction of spreading. While large morphological features of the seafloor such as ridges, trenches, and swells are described deterministically, small-scale features such as abyssal hills are best described stochastically with a model spectrum of the form,

$$
\mathcal{P}(\mathbf{k})=\frac{2 \pi \overline{h^{2}}(\nu-2)}{k_{0} l_{0}}\left[1+\frac{|\mathbf{k}|^{2}}{k_{0}^{2}} \cos ^{2}\left(\phi-\phi_{0}\right)+\frac{|\mathbf{k}|^{2}}{l_{0}^{2}} \sin ^{2}\left(\phi-\phi_{0}\right)\right]^{-\nu / 2},
$$

where $|\mathbf{k}|$ is the wavenumber magnitude and $\phi$ is the wave vector angle with respect to the eastward direction. The free parameters of the model are fitted in a least square sense to the spectrum estimated from observations. These parameters are the rms height of topography $\left(\overline{h^{2}}\right)^{1 / 2}=305 \mathrm{~m}$, the characteristic wavenumbers $k_{0}=2.3 \times 10^{-4}$ 
$\mathrm{m}^{-1}$ and $l_{0}=1.3 \times 10^{-4} \mathrm{~m}^{-1}$ of the principal axes of anisotropy, the azimuth angle $\phi_{0}=320^{\circ}$, and the high wavenumber roll-off slope $\nu=3.5$. Uncertainties in the parameters due to errors of the fit are overwhelmed by uncertainties in correlation between velocity and topography. The implications of those uncertainties in the radiation estimates are discussed below. Fig. 3-8 shows that this representation matches well the data at scales $1 \mathrm{~km}$ and larger which dominate wave radiation. The aspect ratio of the best fit ellipse to the spectrum is 1.8, reflecting a slight anisotropy in the abyssal hills. The rms topographic height in the radiative wavenumber range given by (3.1) is $60 \mathrm{~m}$.

There are no high resolution multi-beam topography data available in the Southeast Pacific region. We rely on the global topography product (http://topex.ucsd.edu) which, in this region, is a combination of satellite bathymetry (Smith and Sandwell, 1997) and available ship soundings (Fig. 3-9). The satellite bathymetry is too coarse to fit the model spectrum, even at the largest scales. Hence we compute a characteristic one-dimensional spectrum as an average over ten different spectra along the ship tracks (Fig. 3-10). The error bar is estimated as a 95\% confidence interval. Spectrum captures the transition from the plateau at topographic scales, larger than about 30$40 \mathrm{~km}$, to a -2.5 roll-off at the smaller scales. At scales smaller than about 4-5 km, which is roughly the resolution of the shipboard data, the topography spectrum is not resolved and drops down rapidly.

We rely on the model of Goff and Jordan (1988) to extrapolate the spectrum down to the whole range of scales necessary for the wave radiation calculation. We fit a one-dimensional form of the model spectrum, obtained by integrating (3.3) along one dimension, to the spectrum estimated from the data at scales which are resolved by the shipboard data. In case of isotropic topography, $k_{0}=l_{0}=k_{*}$, the one-dimensional spectrum is,

$$
\mathcal{P}_{1 D}(k)=\overline{h^{2}} k_{*}^{(\nu-2)}(\nu-2) \cdot B[1 / 2,(\nu-1) / 2] \cdot\left(k_{*}^{2}+k^{2}\right)^{-(\nu-1) / 2},
$$

where $B[1 / 2,(\nu-1) / 2]$ is the beta function. 
If topography is anisotropic then the one-dimensional spectrum has the same form, but the characteristic wavenumber $k_{*}$ is a function of $k_{0}, l_{0}$, and the azimuth angle $\phi_{0}$, i.e. $k_{*}=k_{*}\left(k_{0}, l_{0}, \phi_{0}\right)$. The least-squares estimates of the free parameters of the model are the rms height of topography $\left(\overline{h^{2}}\right)^{1 / 2}=105 \mathrm{~m}$, the characteristic wavenumber $k_{*}=1.8 \times 10^{-4} \mathrm{~m}^{-1}$, and the high wavenumber slope $\nu=3.5$. The model spectrum lies well within the error bar at all resolved scales and provides the required extrapolation to the smaller scales. Scales below $4-5 \mathrm{~km}$ where model is outside the error bar are unresolved by shipboard data and can not be trusted. The rms topographic height is estimated to be about three times smaller in the Southeast Pacific than in the Drake Passage region reflecting a difference in topographic roughness between two regions. This difference results in about an order of magnitude difference in the two topographic spectra, which largely determine wave energy radiation.

\subsection{Energy radiation theory}

A mean geostrophic flow over small-scale finite amplitude topography radiates internal gravity waves. In Chapter 2 we found that the momentum flux associated with the internal waves drives strong inertial oscillations confined to a few hundred meters from the ocean bottom. Numerical simulations show that the inertial oscillations have a limited impact on the energy radiated into the internal wave field: the bottom energy conversion is increased by about $30 \%$ over the steady lee wave generation problem for monochromatic topography. Instead, the inertial oscillations provide a background shear that promotes wave breaking so that large fractions of the radiated wave energy is dissipated within a few hundred meter of the bottom topography. For practical purposes, we will neglect the $30 \%$ correction due to inertial oscillations in the bottom energy conversion estimate. The wave radiation then reduces to the classical lee wave problem discussed by Bell $(1975 \mathrm{a}, \mathrm{b})$. However the inertial oscillation feedback is crucial to our assumption that substantial fraction of radiated energy is locally disipated. In the linear lee wave problem no wave breaking occurs. 


\subsubsection{Lee wave radiation}

Internal lee waves are generated in a stratified fluid when a steady geostrophic flow runs over uneven bottom topography. Bell $(1975 \mathrm{a}, \mathrm{b})$ shows that the energy radiated in steady lee waves, in the limit of small amplitude topography, is given by,

$$
E=\frac{\rho_{0}}{4 \pi^{2}} \int_{-\infty}^{+\infty} \int_{-\infty}^{+\infty} \frac{\left(\mathbf{U}_{o} \cdot \mathbf{k}\right)}{|\mathbf{k}|} \mathcal{P}(\mathbf{k}) \sqrt{\left(\mathbf{U}_{o} \cdot \mathbf{k}\right)^{2}-f^{2}} \sqrt{N^{2}-\left(\mathbf{U}_{o} \cdot \mathbf{k}\right)^{2}} d \mathbf{k}
$$

where $\mathcal{P}(\mathbf{k})$ is the 2-D topography spectrum, $\mathbf{k}=(k, l)$ is the wavenumber vector, $\mathbf{U}_{0}$ is the bottom velocity vector, $N$ is the bottom stratification, $f$ is the Coriolis frequency, and $\rho_{0}$ is a reference density. This result applies for wavenumbers in the radiation range, as given in (3.1), and at small inverse Froude number, $\mathrm{Fr}^{-1}=$ $N h /\left|\mathbf{U}_{0}\right|$, where $h$ is a characteristic topographic height. In Chapter 2 we showed that, for monochromatic topography, the linear theory prediction remains accurate until $F r_{c}^{-1}=0.7$ beyond which energy conversion saturates due to topography blocking effects.

Expression (3.5) predicts that energy radiation at each wavenumber depends on the topographic elevation at that scale and the relative orientation of the velocity and wavenumber vectors. Without loss of generality, we rotate the reference frame to have the velocity vector $\mathbf{U}_{o}$ along the ' $k$ '-axis. Then, $\mathbf{U}_{o} \cdot \mathbf{k}=\left|\mathbf{U}_{o}\right| k$, and the expression (3.5) reduces to,

$$
E=\frac{\rho_{0}\left|\mathbf{U}_{o}\right|}{2 \pi} \int_{-\infty}^{+\infty} d k \sqrt{\left|\mathbf{U}_{o}\right|^{2} k^{2}-f^{2}} \sqrt{N^{2}-\left|\mathbf{U}_{o}\right|^{2} k^{2}} \cdot \frac{1}{2 \pi} \int_{-\infty}^{+\infty} \frac{k}{|\mathbf{k}|} \mathcal{P}(\mathbf{k}) d l,
$$

where $\mathbf{k}=(k, l)$ is now the wavenumber in the reference frame along and across the mean flow. The outer integral on the right hand side depends only on the wavenumber $k$ in the direction of the mean flow vector, and includes contributions from wavenumbers in the radiative range. The inner integral, on the other hand, does not depend on the properties of the mean flow. It depends only on the properties of the topography spectrum and can be integrated over all wavenumbers in the direction across the mean flow velocity vector. It is convenient to define the effective topography spectrum in 
wavenumber $k$ as,

$$
\mathcal{P}_{\text {eff }}(k)=\frac{1}{2 \pi} \int_{-\infty}^{+\infty} \frac{k}{|\mathbf{k}|} \mathcal{P}(\mathbf{k}) d l
$$

Then wave radiation from two-dimensional topography reduces to an equivalent onedimensional wave radiation problem in the direction along the mean flow with the topography spectrum given by $\mathcal{P}_{\text {eff }}(k)$. The energy conversion can now be rewritten in the following nondimensional form as an integral in wavenumber $k$,

$$
\frac{E}{\rho_{0}\left|\mathbf{U}_{0}\right|^{3}}=\frac{1}{\pi} \int_{f /\left|\mathbf{U}_{0}\right|}^{N /\left|\mathbf{U}_{0}\right|} \mathcal{F}(k) \alpha(k)\left(1-\frac{f^{2}}{\left|\mathbf{U}_{0}\right|^{2} k^{2}}\right) d k,
$$

where $\mathcal{F}(k)=\mathcal{P}_{\text {eff }}(k) \mu^{2}(k)$ is the topography inverse Froude number spectrum, $\alpha(k)=k \mu^{-1}(k)$ is the internal wave slope, and $\mu(k)$ is the internal wave vertical wavenumber,

$$
\mu^{2}(k)=k^{2} \frac{N^{2}-\left|\mathbf{U}_{o}\right|^{2} k^{2}}{\left|\mathbf{U}_{o}\right|^{2} k^{2}-f^{2}} .
$$

Equation (3.8) together with the effective topography spectrum defined in (3.7) constitute the building blocks for our estimates of wave radiation from data.

\subsubsection{Simplified theory}

The expression for the energy conversion in (3.8) is quite opaque. Making a few additional assumptions about the shape of the topography spectrum, and the ratio of frequencies $f$ and $N$, the expression can be substantially simplified. As we show below, the additional assumptions are well satisfied in the regions considered in this study.

First, to simplify the shape of topography spectrum we assume that it is isotropic, i.e. $k_{0}=l_{0}=\kappa_{0}$, and internal waves radiate from topography scales such that $|\mathbf{k}|^{2} \gg \kappa_{0}^{2}$, then using (3.3) and (3.7) we obtain,

$$
\mathcal{P}_{\text {eff }}(k)=S k^{-(\nu-1)},
$$


where

$$
S=\overline{h^{2}} \kappa_{0}^{\nu-2}(\nu-2) B(1 / 2, \nu / 2)
$$

This approximation of the effective spectrum captures both the magnitude and the slope of the spectrum at high wavenumbers where wave radiation mostly occurs. Plugging the effective topography spectrum (3.10) into energy conversion expression (3.8) we get,

$$
E=\frac{1}{\pi} \rho_{o} S N\left|\mathbf{U}_{0}\right|^{2} \frac{\left|\mathbf{U}_{0}\right|^{1 / 2}}{f^{1 / 2}} \sqrt{\frac{f}{N}} \cdot \int_{f / N}^{1} t^{2-\nu}\left(1-t^{2}\right)^{1 / 2}\left(1-\frac{f^{2}}{N^{2}} t^{-2}\right)^{1 / 2} d t
$$

where $t$ is the new variable of integration given by,

$$
t=\frac{\left|\mathbf{U}_{0}\right|}{N} k
$$

If we expand the two square roots in their Taylor series, the integral (3.12) can be evaluated analytically. Using the slope of topography spectrum at high wavenumbers $\nu=\frac{7}{2}$ and assuming that $f / N \ll 1$ we get a simplified expression for the energy conversion at the bottom,

$$
E=\frac{1}{\pi} \rho_{o} S N\left|\mathbf{U}_{0}\right|^{2} \cdot \frac{\left|\mathbf{U}_{0}\right|^{1 / 2}}{f^{1 / 2}}\left(\frac{9}{5}-\frac{7}{3} \sqrt{\frac{f}{N}}+O\left(\frac{f^{2}}{N^{2}}\right)\right)
$$

The expression (3.14) can be compared with the equivalent expression for radiation by a monochromatic topography, $E=1 / 2 \rho_{0} h^{2} k N U^{2}$. If we define the mean product of the height squared and the wavenumber $\left.\overline{h^{2} k}\right|_{L W}$ characteristic of the lee wave wavenumber range as,

$$
\left.\overline{h^{2} k}\right|_{L W}=\frac{1}{\pi} \int_{f /\left|\mathbf{U}_{0}\right|}^{N /\left|\mathbf{U}_{0}\right|} k \mathcal{P}_{e f f}(k) d k=\frac{2 S}{\pi} \frac{\left|\mathbf{U}_{0}\right|^{1 / 2}}{f^{1 / 2}}\left(1-\sqrt{\frac{f}{N}}\right),
$$


then expression (3.14) takes the form,

$$
E=\left.\frac{1}{2} \rho_{o} \overline{h^{2} k}\right|_{L W} N\left|\mathbf{U}_{0}\right|^{2} \cdot \frac{\left(\frac{9}{5}-\frac{7}{3} \sqrt{\frac{f}{N}}+O\left(\frac{f^{2}}{N^{2}}\right)\right)}{\left(1-\sqrt{\frac{f}{N}}\right)}
$$

To the leading order energy radiation by a multichromatic topography can be understood as radiation from a mono-chromatic bump with topographic characteristics

given by $\left.\overline{h^{2} k}\right|_{L W}$. However, as opposed to the energy radiation from monochromatic topography which depends quadratically on velocity magnitude $\left|\mathbf{U}_{0}\right|^{2}$, multichromatic energy radiation is proportional to $\left|\mathbf{U}_{0}\right|^{5 / 2}$ where the dependence on velocity is determined by the slope of the topographic spectrum in the wave number range associated with lee wave radiation.

\subsection{Results}

The wave radiation expressions in (3.8) and (3.14) are now used to estimate the generation and dissipation of internal gravity waves from the velocity and stratification data discussed above. Energy radiation is estimated for each station along the sections. It is assumed that the topography characteristics are uniform along each section for the two regions considered.

\subsubsection{Effective topography spectrum}

We estimate the effective spectrum defined in (3.7) using the 2-D model spectrum in (3.3) and integrating it over all wavenumbers across the direction of the mean flow. In the Drake Passage region the 2-D spectrum is estimated directly from data and all parameters of the model spectrum are defined by a least square fit. In the Southeast Pacific, only 1-D topographic sections are available, and the 2-D spectral representation i.e. the degree of anisotropy can not be uniquely determined; we will resort to estimate the uncertainty associated with plausible ranges in anisotropy.

In order to estimate $P_{\text {eff }}$, we proceed as follows. First, we assume that the mean 
geostrophic flow can come at any angle with respect to the topography. The KE in the geostrophic velocity field is dominated by transient eddies, whose velocity direction is very variable and likely to span the whole $360^{\circ}$ over a few eddy turn-over times. Rather than estimating energy radiation for a particular velocity realization we estimate radiation for two limiting cases, i.e. for a flow going across and along the major axis of the topographic spectrum. The two estimates provide the lower and the upper limits of the wave energy radiation estimate and, thus, define the uncertainty due to the variable and unknown orientation between the mean flow and topography. Drake Passage estimates of the effective spectrum are shown in Fig. 3-11. Both spectra have a -2.5 roll-off in the lee wave radiation wavenumber range. Weak anisotropy in topography, 1.8, results in about a factor of 3 difference between the lower and the upper limits of the effective spectrum estimate.

In the Southeast Pacific we must further address the issue of how sensitive results are to the undetermined degree of anisotropy in topography. As a starting point, we assume that its aspect ratio is similar to the one in the Drake Passage region, $a \simeq 1.8$. In both the Southeast Pacific and Drake Passage regions topography at scales larger than 50-100 km, is dominated by ridges with similar characteristics. Since topographic features formed at the ridged crests tend to elongate perpendicular to the direction of spreading in a self-similar fashion (Goff and Jordan, 1988), it seems reasonable to assume that the spectral roll-offs are similar in the two regions. Nevertheless, we also estimate the radiation for an isotropic topography to quantify the effect of uncertainties in anisotropies in topography on the radiation calculations.

Estimates of the effective spectrum for the Southeast Pacific are shown in Fig. 3-11. The lower and the upper radiation limits correspond, respectively, to the mean flow along the short principal axis of the ellipse $\left(k_{*}, a^{-1} k_{*}\right)$ and along the long principal axis of the ellipse $\left(a k_{*}, k_{*}\right)$. The lower and the upper limits are different by a factor of about 10-12 in lee wave radiation range. The greater degree of uncertainty in the Southeast Pacific region results from the uncertainty in both the mean flow orientation and the length of the principal axes of anisotropy. The lower limit of the Drake Passage region spectrum in about 1.5 times greater that the upper limit of the 
Southeast Pacific region spectrum implying that the topographic roughness is systematically greater in Drake Passage at all topographic scale and in both directions of the principal axes.

\subsubsection{Inverse Froude number estimate}

Idealized numerical simulations with monochromatic topography show that the character of the radiated waves and the overall magnitude of energy radiated depend on the value of the inverse Froude number, $\mathrm{Fr}^{-1}$. The bottom energy conversion is shown to increase quadratically with inverse Froude number consistent with linear theory, for $\mathrm{Fr}^{-1} \leq \mathrm{Fr}_{c}^{-1}=0.7$, where $\mathrm{Fr}_{c}^{-1}$ is the critical inverse Froude number. When $\mathrm{Fr}^{-1}$ exceeds the critical value $\mathrm{Fr}_{c}^{-1}$ some fraction of the mean flow becomes blocked by topography and energy conversion saturates. The interpretation is that the stagnant fluid in the trough acts to reduce the effective topography seen by the mean flow. This saturation is included in the energy conversion estimate for the Southern Ocean by limiting energy conversion for inverse Froude numbers larger that the critical value. The inverse Froude number that characterizes the whole range of topographic wavenumbers is estimated from the inverse Froude number spectrum as,

$$
F r^{-1}=\sqrt{\frac{2}{\pi} \int_{f /\left|\mathbf{U}_{0}\right|}^{N /\left|\mathbf{U}_{0}\right|} \mathcal{F}(k) d k}
$$

In the limit of monochromatic topography the vertical scale of the waves is $\simeq N /\left|\mathbf{U}_{\mathbf{0}}\right|$ and this definition of inverse Froude number reduces to the definition $N h /\left|\mathbf{U}_{\mathbf{0}}\right|$ used to describe 2-D idealized simulations in Chapter 2. Here, we use expression (3.17) to estimate $\mathrm{Fr}^{-1}$ for the multichromatic topography in the Drake Passage and the Southeast Pacific regions.

An example of inverse Froude number spectrum $\mathcal{F}(k)$ computed using the Drake Passage topography spectrum and the mean flow characteristics in the Polar Front of the ACC is shown in Fig. 3-12. The $\mathcal{F}(k)$ spectrum is dominated by large topographic scales because the ratio of the topographic height to the vertical scale of the radiated waves grows with scale: scales with the lowest frequency $f$ have infinite vertical 
wavenumber and the inverse Froude number spectrum becomes infinite. The result is correct in the sense that large-scale lee waves are super-critical with respect to topography (their ray paths are nearly horizontal) and hence are likely to become nonlinear. However these waves do not contribute much to upward energy radiation. To estimate $\mathrm{Fr}^{-1}$ for the entire range of topographic scales we include only those waves whose vertical group velocity is large enough to radiate $1 \mathrm{~km}$ above the bottom within 10 days, which is a characteristic eddy time scale. These are the waves that can efficiently radiate energy far above the bottom and sustain enhanced energy dissipation observed in the bottom $1 \mathrm{~km}$. The other waves can contribute to bottom dissipation, but not to radiation.

Fig. 3-13 shows inverse Froude numbers estimated from (3.17) for the sections across Drake Passage and in the Southeast Pacific. The Southeast Pacific section is characterized by smaller values of inverse Froude number of about $\mathrm{Fr}^{-1}=0.05-0.3$ mostly due to the lower energy levels of effective topography spectrum. In Chapter 2 we showed that for this $\mathrm{Fr}^{-1}$ range radiation is mostly in the form of quasi-stationary lee waves. In the Drake Passage region, however, the inverse Froude number spans the range from about $\mathrm{Fr}^{-1}=0.2-0.4$ south of $60.5^{\circ} \mathrm{S}$ to roughly $\mathrm{Fr}^{-1}=0.5-1.0$ north of it. These Froude numbers correspond to the time-dependent wave radiation regime associated with vigorous inertial oscillations and wave breaking in the bottom $1 \mathrm{~km}$. $\mathrm{Fr}^{-1}$ is lower in the South ACC Front than in the Polar Front mostly because of the lower values of stratification frequency in the southern part of the Drake Passage region.

To summarize, inverse Froude numbers in the two regions of the Southern Ocean considered in this study vary from essentially zero to $\mathrm{Fr}^{-1}=1.0$, corresponding to sub-critical and critical topography regimes. In idealized numerical simulations with monochromatic topography this range of inverse Froude number spans the transition from the radiation of stationary lee waves to the radiation of time-dependent waves. The numerical experiments showed that the bottom value of energy flux is well predicted by the linear theory for both wave radiation regimes until $\mathrm{Fr}^{-1}$ reached $F r_{c}^{-1}$. Radiation levels did not further increase for $\mathrm{Fr}^{-1} \geq F r_{c}^{-1}$. Inverse Froude number 
estimated from observations exceeds this critical value for the stations in the Polar Front of the ACC.

\subsubsection{Energy radiation estimate}

We estimate energy radiation in both regions using effective topography spectra, bottom velocity and stratification. In each station where the inverse Froude number exceeds its critical value bottom energy radiation is adjusted to account for the wave saturation effect,

$$
E= \begin{cases}E\left(F r^{-1}\right) & F r^{-1} \leq F r_{c}^{-1} \\ E\left(F r^{-1}\right)\left(\frac{F r_{c}}{F r}\right)^{-2} & F r^{-1}>F r_{c}^{-1}\end{cases}
$$

where $E\left(F r^{-1}\right)$ is given by (3.8). This correction reduces the upper limit of the energy conversion estimate by about $25 \%$ only in the Polar Front in Drake Passage. Elsewhere $\mathrm{Fr}^{-1}$ does not exceed the critical value $\mathrm{Fr}_{c}^{-1}$.

We report four different calculations: (I) We compute $E$ from the full expression (3.8) and the anisotropic spectrum (3.3). The upper and lower values corresponding to flow along the major and minor axis of the topographic spectrum are reported, (II) We repeat the estimate assuming that the spectra are isotropic, (III) Finally we use the simplified expression in (3.14). Energy radiation estimates for the section in the Drake Passage region are shown in Fig. 3-14. Energy conversion in Drake Passage is highly non-uniform along the section. Its spatial variability is dominated by the bottom velocity distribution and has two maxima corresponding to the Polar Front

and the South ACC Front of the ACC. Values of energy radiation averaged along the section are in the range $15-31 \mathrm{~mW} \mathrm{~m}^{-2}$ where the lower and the upper limits correspond respectively to the mean flow along and across topographic anisotropy.

Energy conversion estimates computed using an isotropic spectrum and the simpler expression (3.14) fall in between the lower and the upper limits of the wave energy radiation estimate south of Drake Passage and close to the upper limit in the north. The isotropic spectrum calculation and the simplified theory result agree remarkably well in both regions supporting the assumptions made to simplify and 
evaluate the integral expression. Section averaged energy radiation estimated using isotropic spectrum is $25 \mathrm{~mW} \mathrm{~m}^{-2}$ and using the simplified expression (3.14) is $27 \mathrm{~mW}$ $\mathrm{m}^{-2}$.

Variations in the energy radiation estimate for an anisotropic spectrum are due to variation in the direction of the mean flow. We expect the eddy velocity to span all directions over time. Therefore, the best estimate of the time average radiation is the mean between 15 and $31 \mathrm{~mW} \mathrm{~m}^{-2}$, i.e. $23 \mathrm{~mW} \mathrm{~m}^{-2}$.

We make the same three estimates along the section in the Southeast Pacific region (Fig. 3-15). Energy radiation estimates are more than an order of magnitude smaller mostly due to lower energy levels in topography spectrum and, partly, because of lower values of the bottom stratification and velocity. The energy radiation estimated using anisotropic spectrum and averaged along the section is in the range $0.4-3.3 \mathrm{~mW} \mathrm{~m}^{-2}$. The wide range in this estimate reflects the uncertainty in anisotropy, which adds to the mean flow orientation uncertainty discussed in the previous estimate. Estimates based on an isotropic spectrum and the simplified theory (3.14) produce respectively $1.2 \mathrm{~mW} \mathrm{~m}^{-2}$ and $1.4 \mathrm{~mW} \mathrm{~m}^{-2}$ for the section averaged energy radiation. Radiation is increased in the core of the ACC and drops down essentially to zero on its flanks. Once again, the mean value, $1.8 \mathrm{~mW} \mathrm{~m}^{-2}$, is our best estimate of the time average radiation spanning all possible velocity orientations.

Wave energy radiation estimates from the linear theory agree very well with the magnitudes and spatial distribution of turbulent energy dissipation observed directly in the Southern Ocean (Naveira-Garabato et al., 2004). This result suggest that the bulk of enhanced mixing observed in the Southern Ocean can be sustained by geostrophic flow impinging on small-scale topography.

\subsection{Multichromatic topography simulations}

The amount of observed energy dissipation and its spatial variation between the two regions seems to be well captured by the linear energy radiation estimate. However, the energy radiated by internal waves might not be all dissipated locally. Some 
fraction of that energy can radiate away in low-modes and dissipate in remote regions. Idealized numerical simulations with monochromatic bottom topography show that about $30 \%$ of energy radiated by waves generated by geostrophic flows dissipates locally within $1 \mathrm{~km}$ above the bottom. In this section, we show that the result carries over to multichromatic topography.

\subsubsection{Experiment setup}

We use the nonhydrostatic configuration of the MIT general circulation model (MITgcm) (Marshall et al., 1997). The numerical setup is similar to that used in the idealized simulations described in Chapter 2. The domain is 2-D, horizontally periodic with $L_{x} \times H_{z}=10 \mathrm{~km} \times 7 \mathrm{~km}$, somewhat larger than in Chapter 2 to allow for different topographic wavenumbers. We use a resolution of $\Delta x=16.6 \mathrm{~m}$ in the horizontal and $\Delta z=10 \mathrm{~m}$ in the vertical which gradually stretches to $\Delta z=300 \mathrm{~m}$ in the region from $2 \mathrm{~km}$ to $7 \mathrm{~km}$ above the bottom. Boundary conditions are a sponge layer between 2 and $7 \mathrm{~km}$ above the bottom to absorb waves that do not break in the bottom $1 \mathrm{~km}$, and free-slip condition at the bottom. No-slip boundary condition is expected to reduce wave radiation by about $20-30 \%$ based on two-dimensional idealized simulations with no-slip boundary condition discussed in Chapter 2. A depth-independent mean flow $U_{G}=0.1 \mathrm{~m} \mathrm{~s}^{-1}$ is forced by adding a body force $f U_{G}$ to the meridional momentum equation representing a barotropic pressure gradient which balances mean flow geostrophically at all depths.

The bottom topography used in the simulations is randomly generated to have the same spectrum as in the observations, i.e. the 1-D model spectrum in (3.10) with the spectrum amplitude chosen to determine $\mathrm{Fr}^{-1}$ as defined in (3.17). Topography includes horizontal scales in the characteristic lee wave radiation range which spans scales roughly from $600 \mathrm{~m}$ to $6 \mathrm{~km}$ and greatly exceeds spectrum roll-off wavenumber $\kappa_{0}=1.8 \times 10^{-4} \mathrm{~m}^{-1}$ required to use model spectrum (3.10). The model spectra of the observed topography and randomly generated simulation spectra corresponding to the Drake Passage and the Southeast Pacific regions are shown in Fig. 3-16. In

the simulation of the Southeast Pacific region the topography amplitude is factor of 
10 smaller compared to the Drake Passage simulation, as estimated from topographic data.

\subsubsection{Results}

Snapshots of the wave zonal velocity component for the Drake Passage and the Southeast Pacific regions after 5 days of simulations are shown in Fig. 3-17 and Fig. 3-18 respectively. The Drake Passage simulation is characterized by radiation of waves with large amplitude of about $0.1 \mathrm{~m} \mathrm{~s}^{-1}$ which is comparable to the magnitude of the mean flow. As waves radiate away from topography, they break and their amplitude drops by an order of magnitude within the bottom $1 \mathrm{~km}$. The wave fields both close to the topography and in the far field are highly multichromatic and time-dependent. In the Southeast Pacific simulation, where the topography spectrum is an order of magnitude smaller, waves have a lower amplitude and, more importantly, do not decay significantly with height. Waves radiate freely away from topography until they reach the sponge layer at the upper boundary and dissipate. The wave field is dominated by waves with horizontal scale of roughly $3 \mathrm{~km}$ with a weaker multichromatic background field due to different topographic scales. Waves are stationary and have scales consistent with linear lee wave theory.

The time evolution of the zonally averaged velocity is shown in Fig. 3-19 and Fig. 3-20 for the Drake Passage and the Southeast Pacific regions respectively. The Drake Passage simulation is characterized by vigorous inertial oscillations which develop spontaneously and reach a magnitude comparable to the mean flow within the first 3-5 days. In the Southeast Pacific simulation, however, there are no inertial oscillations for the whole period of simulation. This result is consistent with the resonant feedback mechanism described in Chapter 2. Large amplitude internal waves in the Drake Passage simulation, drive a large momentum flux divergence and trigger inertial oscillations at the bottom. Inertial oscillations in turn modify the wave generation process and produce time dependent internal waves which can effectively reinforce inertial oscillations. The combination of large amplitude waves and inertial oscillations leads to a new statistically steady state characterized by time dependent 
waves and bottom intensified inertial oscillations. In the Southeast Pacific, wave radiation is well described by the linear lee wave theory due to the lower inverse Froude number in this region.

Vertical profiles of energy dissipation rate diagnosed from the simulations are shown in Fig. 3-21. Energy dissipation is one to two orders of magnitude larger in Drake Passage than in the Southeast Pacific. Dissipation is significantly enhanced in the bottom several hundred meters in the Drake Passage and nearly uniform in the southeast Pacific. Total energy dissipation integrated in the bottom $1 \mathrm{~km}$ is 18 $\mathrm{mW} \mathrm{m} \mathrm{m}^{-2}$ and $1 \mathrm{~mW} \mathrm{~m}^{-2}$ in the two regions. Linear theory energy radiation values corresponding to these two simulations are $28 \mathrm{~mW} \mathrm{~m}^{-2}$ and $6 \mathrm{~mW} \mathrm{~m}^{-2}$. A greater fraction of radiated energy dissipates locally in the Drake Passage, close to 65\%, than in the Southeast Pacific simulation, close to 15\%. Both the vertical profiles and the total value of energy dissipation are consistent with direct observations (NaveiraGarabato et al., 2004).

\subsection{Conclusions}

Recent estimates from LADCP observations show enhanced turbulent mixing in the Southern Ocean associated with internal wave breaking which is typically concentrated in the bottom $1 \mathrm{~km}$ and has significant spatial variations. In this study we tested the hypothesis that this mixing can be sustained by internal waves generated by geostrophic eddies flowing over small-scale bottom topography. We applied linear wave radiation theory to the bottom topography, velocity and stratification data from the Southeast Pacific and the Drake Passage regions characterized respectively by low and high rates of abyssal mixing. We showed that the estimated energy radiation and its spatial variation are consistent with the observations. Using numerical simulations with topographic characteristics representative of these two regions we

confirmed that significant fraction of energy radiated by internal waves can dissipate locally and sustain the energy dissipation observed in the bottom $1 \mathrm{~km}$.

Section averaged wave energy radiation is estimated to be $0.4-3.3 \mathrm{~mW} \mathrm{~m}^{-2}$ in 
the Southeast Pacific region and $15-31 \mathrm{~mW} \mathrm{~m}^{-2}$ in the Drake Passage region. The lower and the upper limits of these two estimates are determined by the uncertainty in the orientation of the mean flow with respect to anisotropy in topography: mean flows in the direction across small-scale topographic features radiate more energy than flows along. As only 50\% of energy radiation estimated from linear lee wave theory dissipates locally, our best estimate for local energy disipation is less than $1 \mathrm{~mW} \mathrm{~m}^{-2}$ in the Southeast Pacific and $12 \mathrm{~mW} \mathrm{~m}^{-2}$ in the Drake Passage region. These estimates are on the same order as the $1 \mathrm{~mW} \mathrm{~m}^{-2}$ and $10 \mathrm{~mW} \mathrm{~m}^{-2}$ of energy dissipation estimated from observations in these two regions (Naveira-Garabato et al., 2004).

The results show that energy radiation varies substantially across the ACC. In Drake Passage it is strongly dominated by the Polar Front and the South ACC Front of the ACC. The South ACC Front has lower energy radiation, mostly due to the lower values of stratification. In the Southeast Pacific region stratification does not change significantly along the section and the energy radiation tracks variations in the velocity field. It is largest in the core of the ACC and drops down essentially to zero on its flanks.

Our analysis suggests that the substantial difference in energy radiation between these two regions results partly from small differences in velocity and stratification and mostly from differences in topographic roughness. If applied to the same topographic spectrum energy radiation in the two regions would differ by only about a factor of 3 , because velocity and stratification are similar in the two regions. The topographic spectrum, however, is estimated to be an order of magnitude larger in the Drake Passage region, resulting in wave radiation more than 10 times larger than in the Southeast Pacific region.

Inverse Froude numbers are estimated to be in the range 0.05-0.3 and 0.2-1.0 in the Southeast Pacific and the Drake Passage regions respectively. According to idealized numerical simulations wave radiation at inverse Froude numbers $\mathrm{Fr}^{-1}<0.7$ is well described by the linear theory. We ran numerical simulations representative of topography and flows in the two regions. In Drake Passage radiation was strongly 
nonlinear and generated strong inertial shear and wave breaking. About $65 \%$ of the total energy radiated was dissipated within $1 \mathrm{~km}$ of the topography. In the Southeast Pacific region, radiation was in the form of linear lee waves and only $15 \%$ of the radiated energy was actually dissipated.

Linear theory and numerical simulations show that the wave energy radiation is proportional to the bottom value of kinetic energy in geostrophic motions. Ferrari and Wunsch (2008) show that $80-90 \%$ of the KE of the ocean is in geostrophic eddies generated by instabilities of the mean currents. This implies that wave radiation is largely maintained by geostrophic eddies rather than by mean flows. The results further show that wave radiation and subsequent breaking are very sensitive to small-scale topographic roughness and orientation of bottom velocity with respect to anisotropy in topography. Since topographic roughness is spatially variable in the ocean and geostrophic velocities change on the eddy turnover time scale, wave radiation and dissipation at the ocean bottom are predicted to be both spatially and temporally variable.

Our results suggest that there is a direct correlation between small-scale dissipation rates and the magnitude of the geostrophic eddy velocity. Such a correlation could be investigated with mooring data. Another avenue for future research is to study the degree to which wave radiation extracts energy from the geostrophic eddy field. 


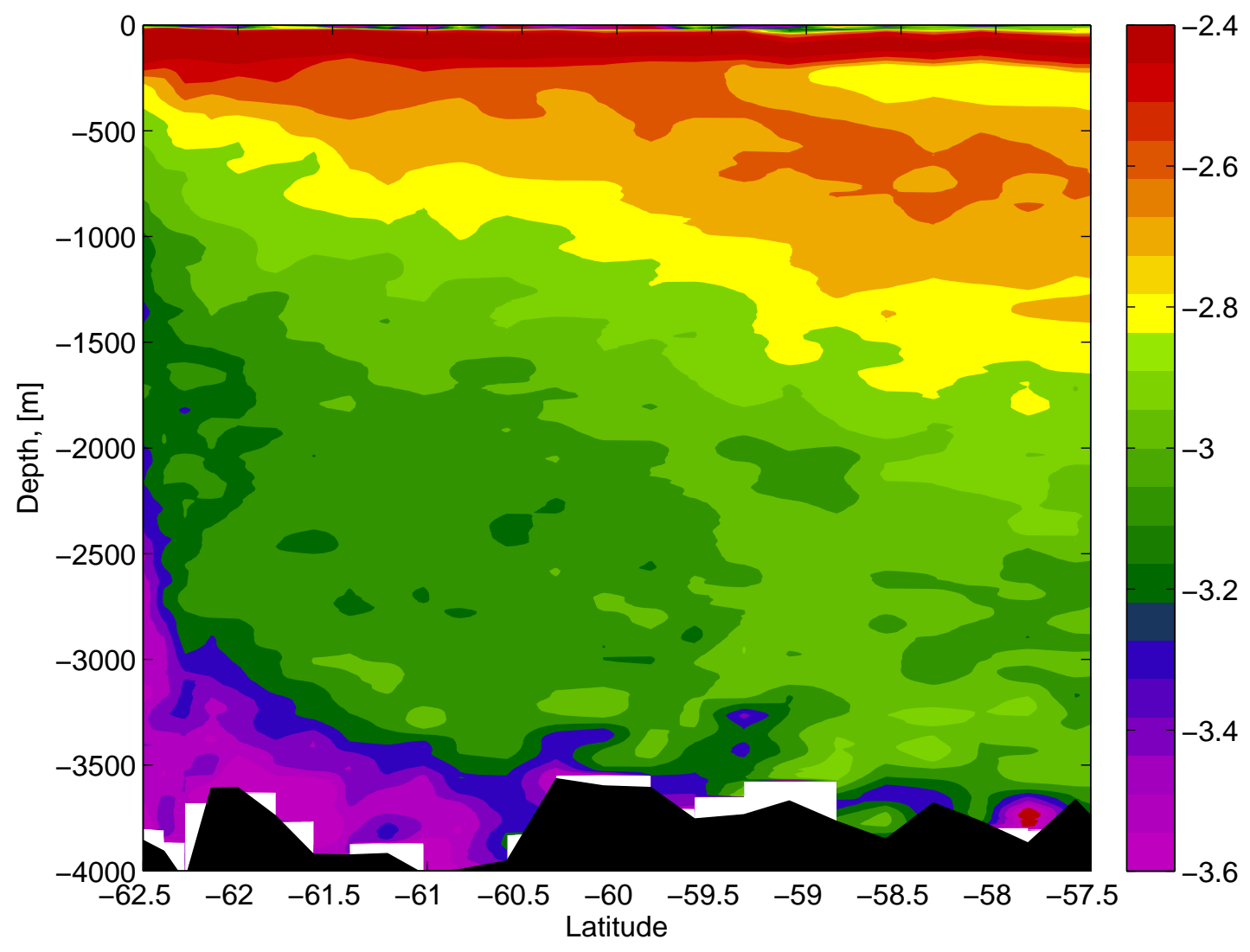

Figure 3-1: Buoyancy frequency $\left(\mathrm{s}^{-1}\right)$ in logarithmic scale from the ALBATROSS section, Drake Passage. 




Figure 3-2: Flow speed $\left(\mathrm{m} \mathrm{s}^{-1}\right)$ from the ALBATROSS section, Drake Passage. 


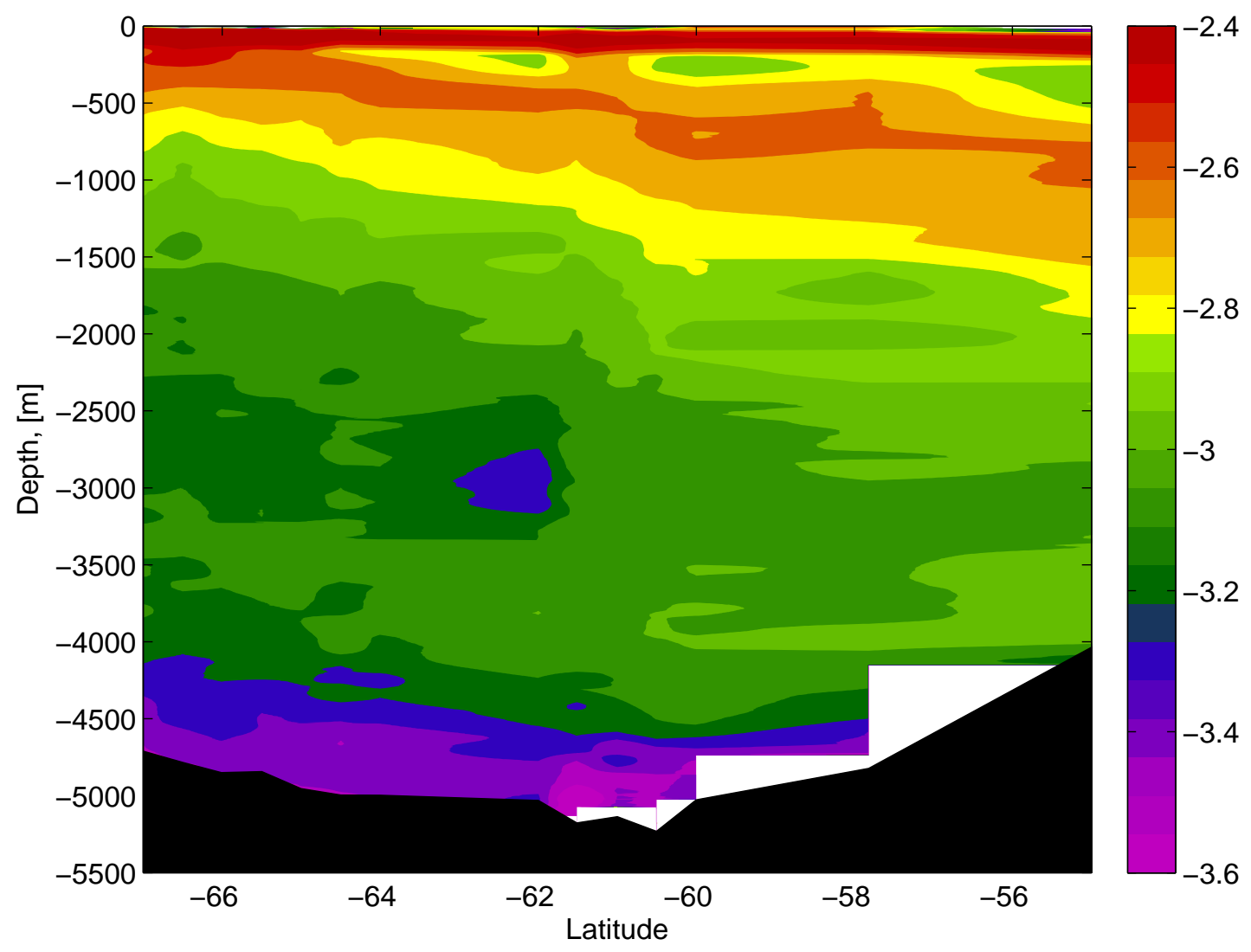

Figure 3-3: Buoyancy frequency $\left(\mathrm{s}^{-1}\right)$ in logarithmic scale from the P18 section, Southeast Pacific. 


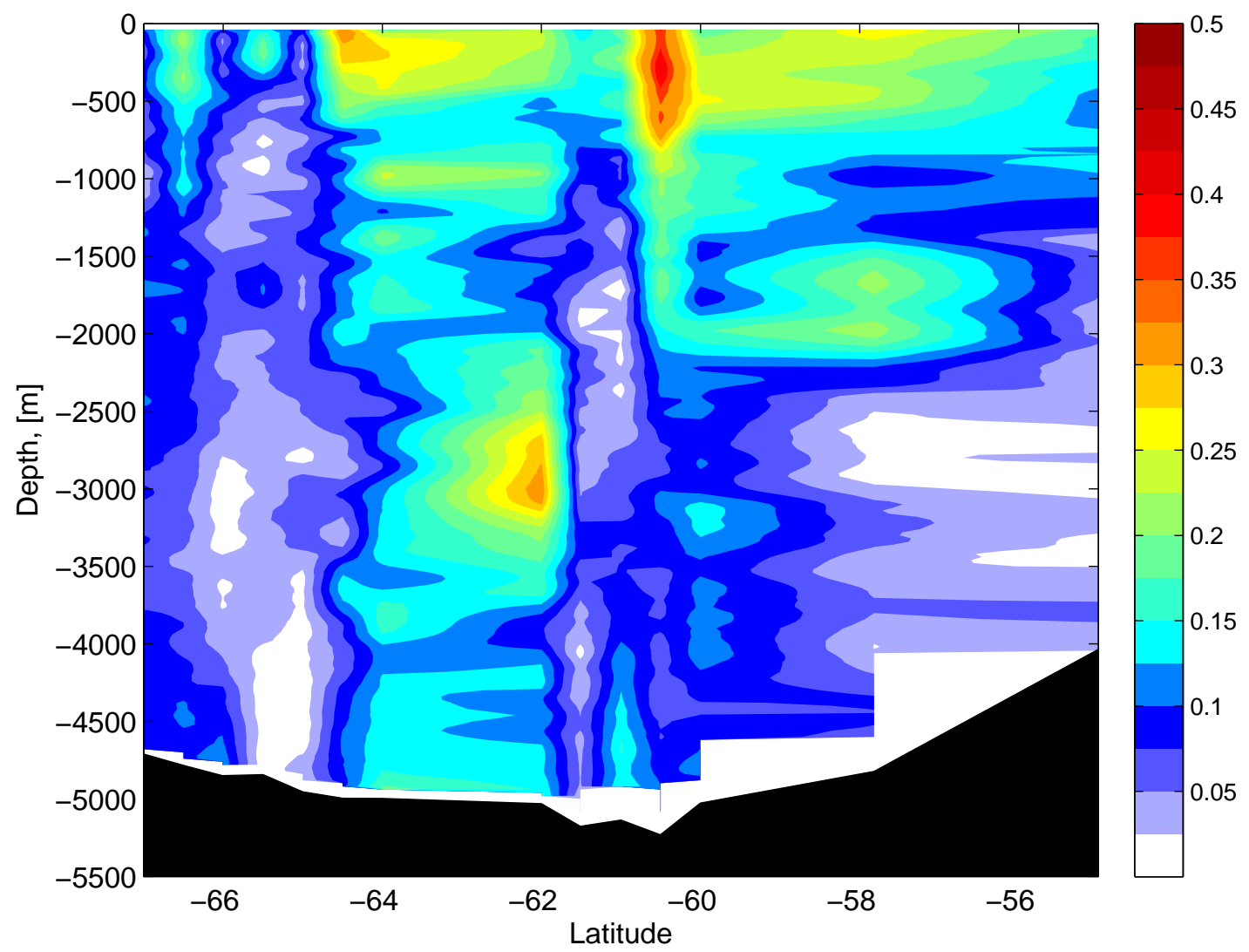

Figure 3-4: Flow speed $\left(\mathrm{m} \mathrm{s}^{-1}\right)$ from the P18 section, Southeast Pacific. 


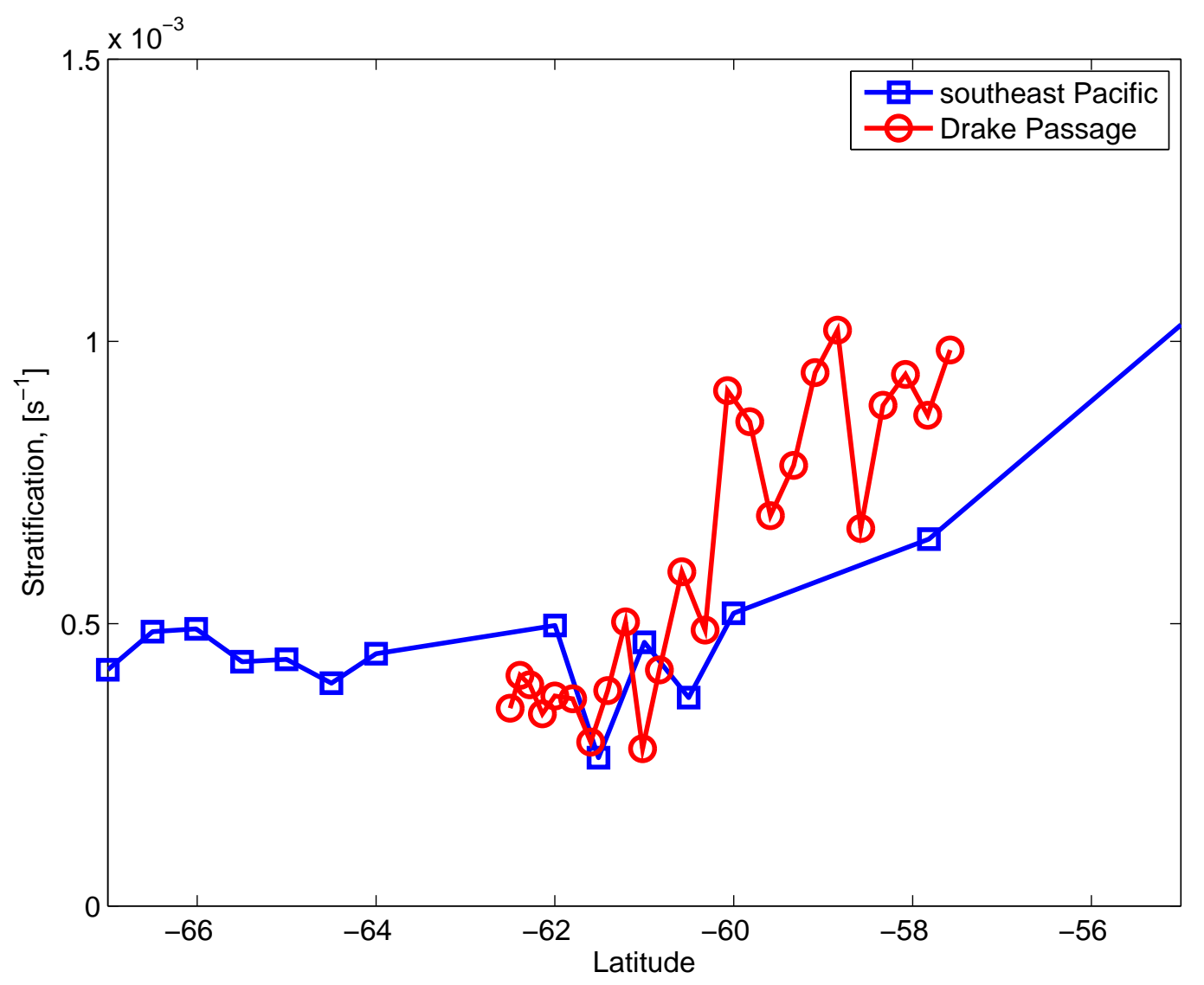

Figure 3-5: Bottom stratification $\left(\mathrm{s}^{-1}\right)$ for the Drake Passage and the Southeast Pacific regions. 


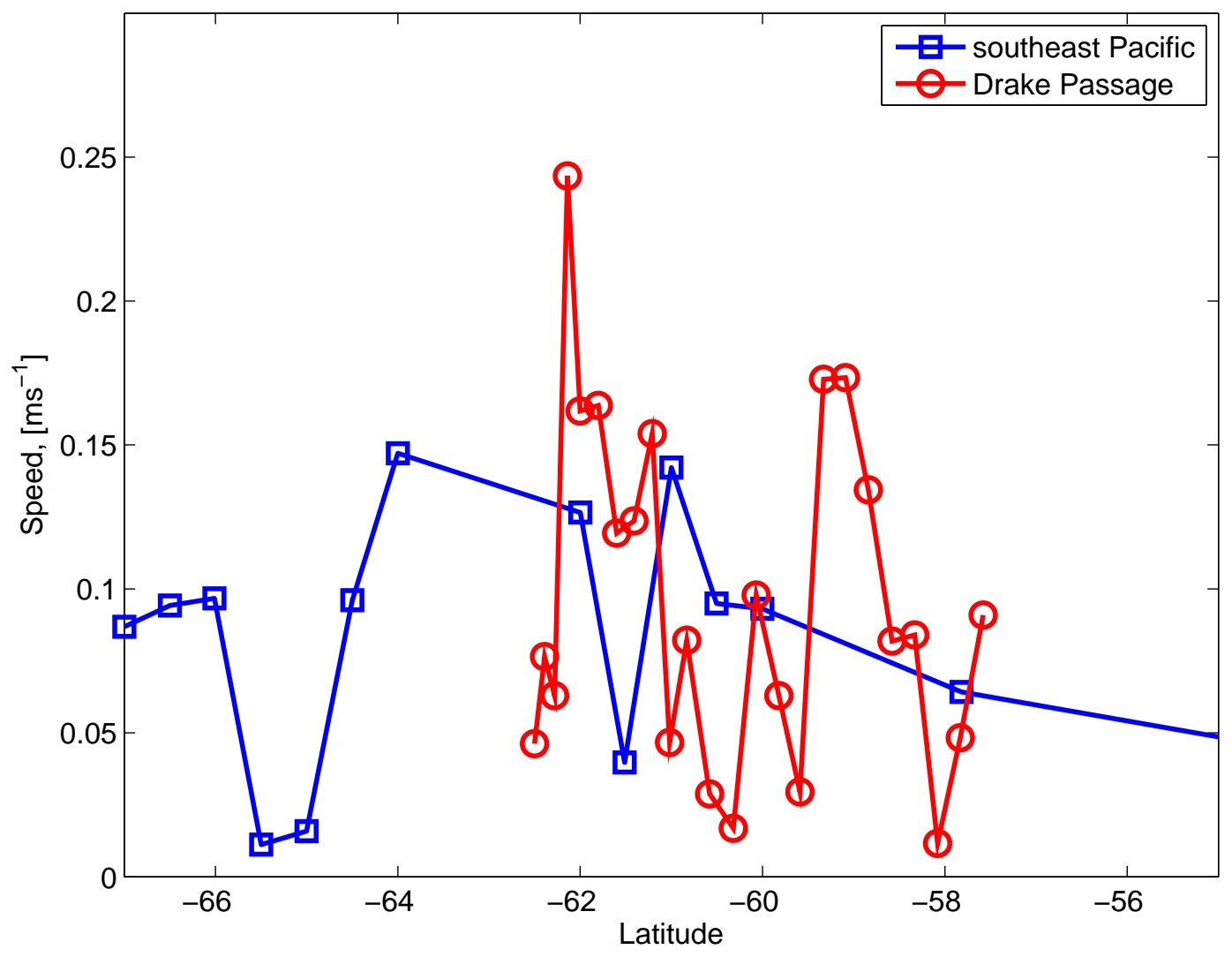

Figure 3-6: Bottom speed $\left(\mathrm{m} \mathrm{s}^{-1}\right)$ for the Drake Passage and the Southeast Pacific regions. 


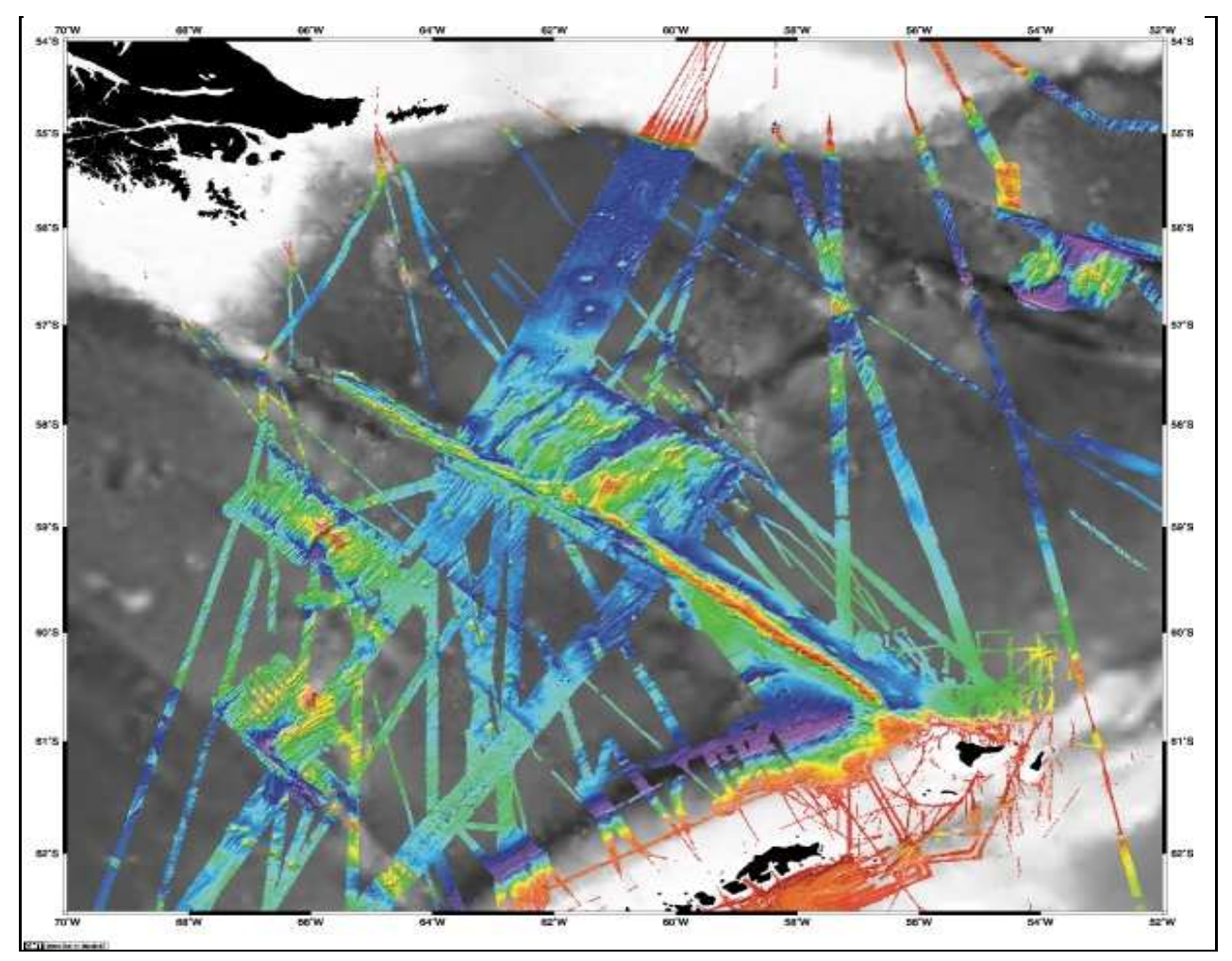

Figure 3-7: Multi-beam topography data (British Antarctic Survey) for the Drake Passage region superimposed on the bathymetry data from satellite altimetry (Smith and Sandwell, 1997). 


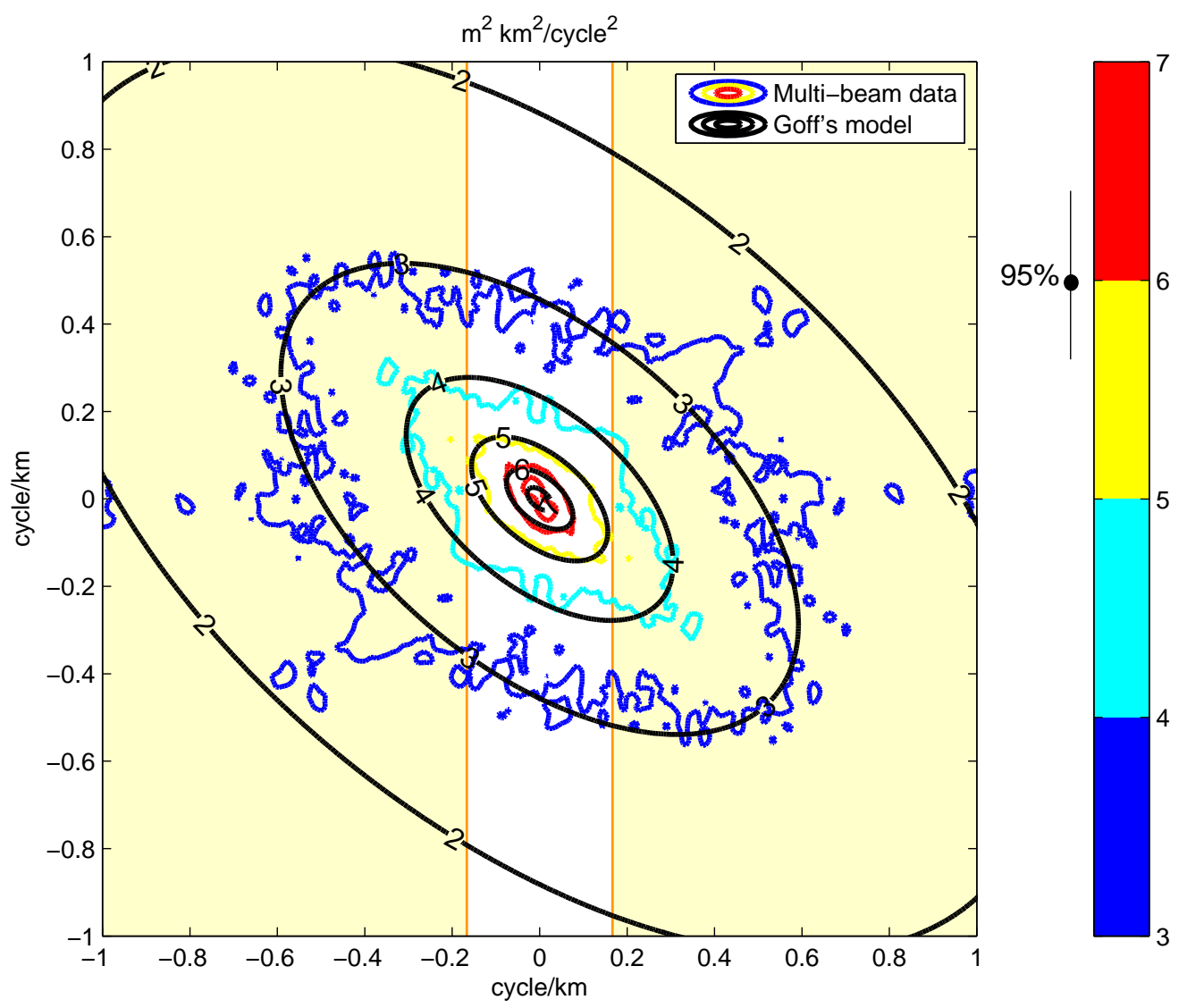

Figure 3-8: Two-dimensional topography spectrum estimated using multi-beam topography data from the Drake Passage (colored lines) and fitted with model spectrum (Goff and Jordan, 1988) (black lines); characteristic wavenumber range for lee wave radiation (yellow). 


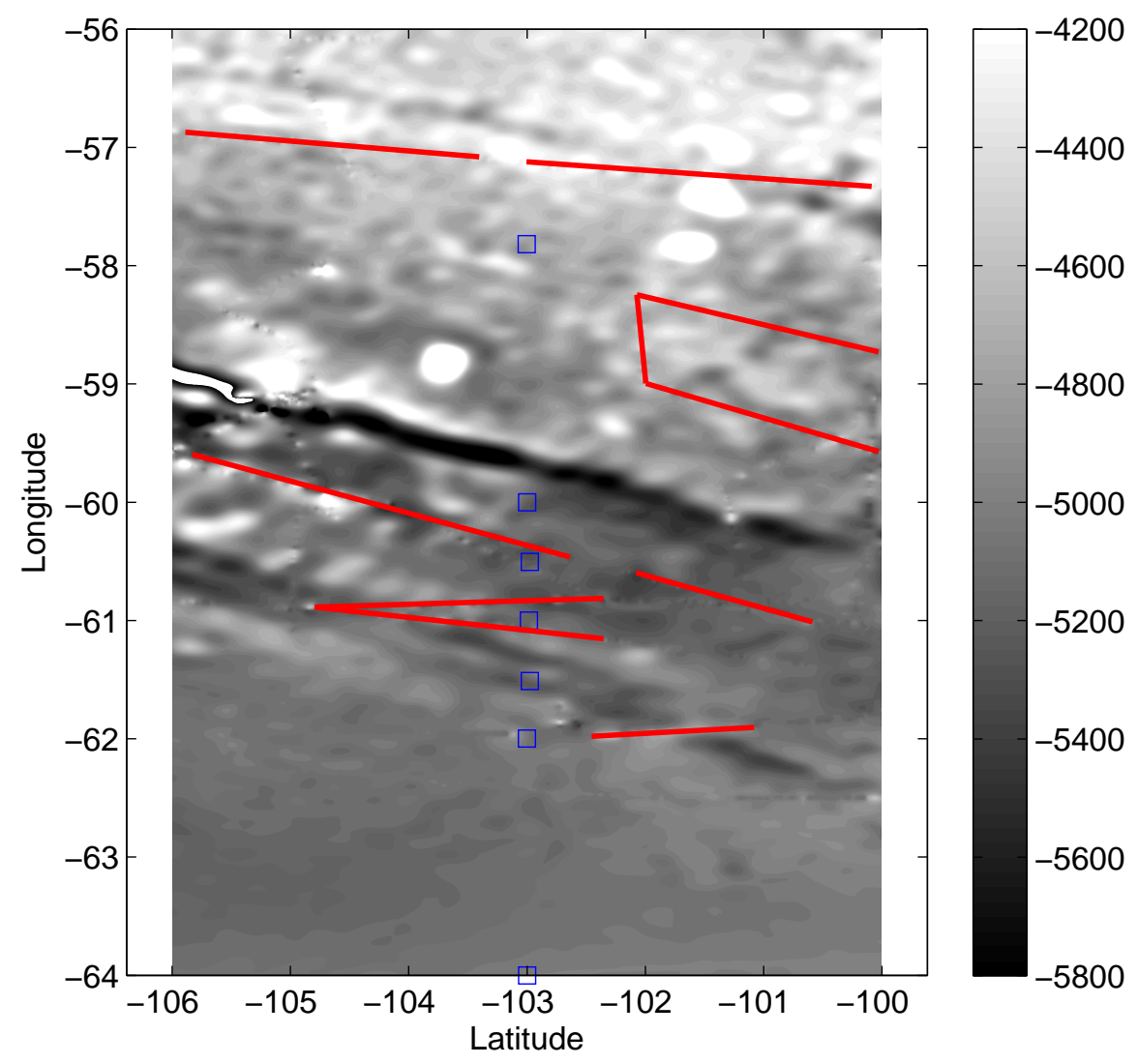

Figure 3-9: Satellite bathymetry of the Southeast Pacific region (m); LADCP stations along P18 section (blue), shipboard topographic data (red). 


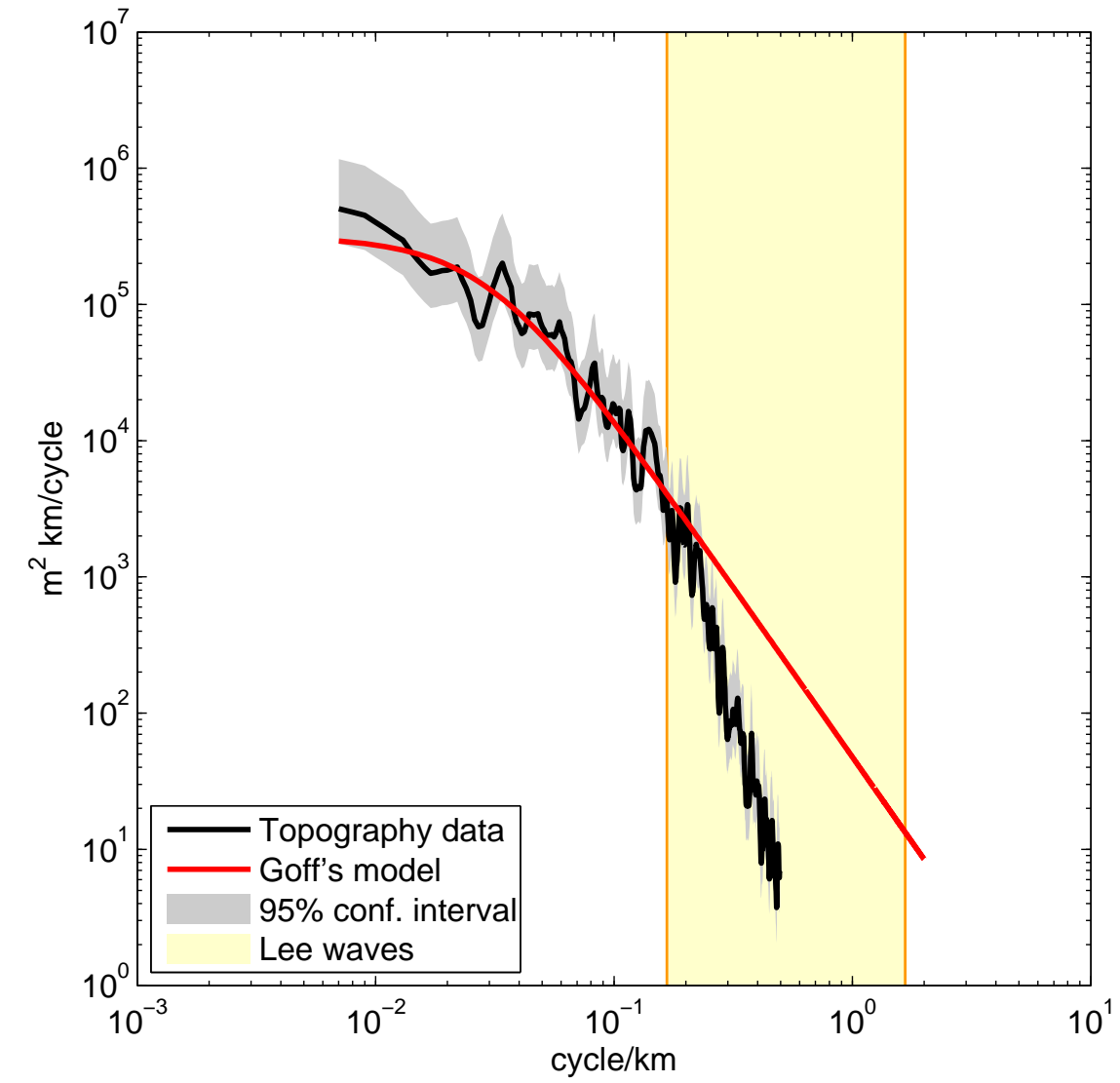

Figure 3-10: One-dimensional topography spectrum estimated along ship tracks from the combination of satellite bathymetry and shipboard topography. 




Figure 3-11: Effective topographic spectra for the Drake Passage region (red) and the Southeast Pacific region (blue) corresponding to the lower (dashed) and the upper (solid) limits of wave energy radiation. 


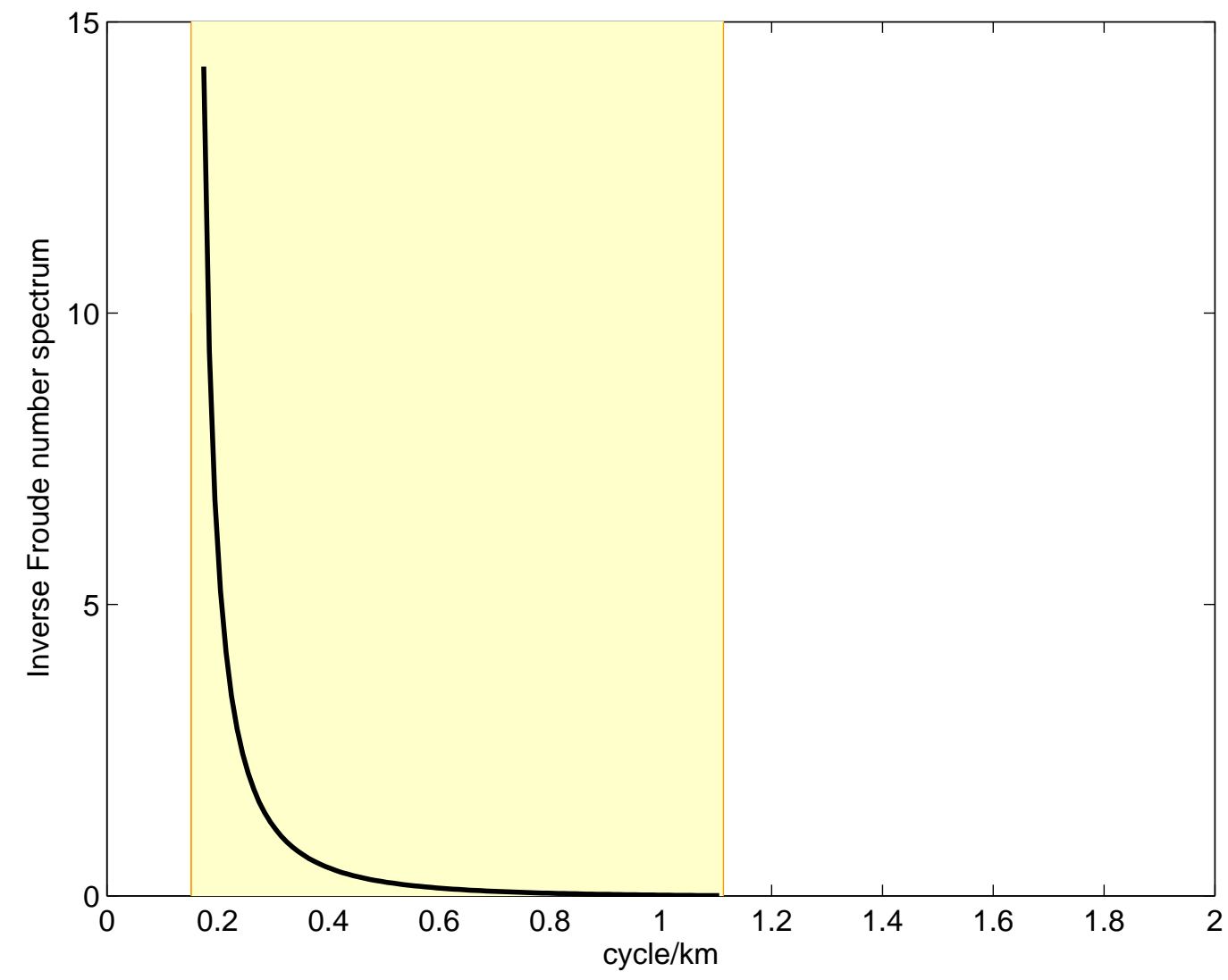

Figure 3-12: Inverse Froude number spectrum (black) for a station in the Polar Front of the ACC in the Drake Passage region, lee wave radiation range (yellow). 


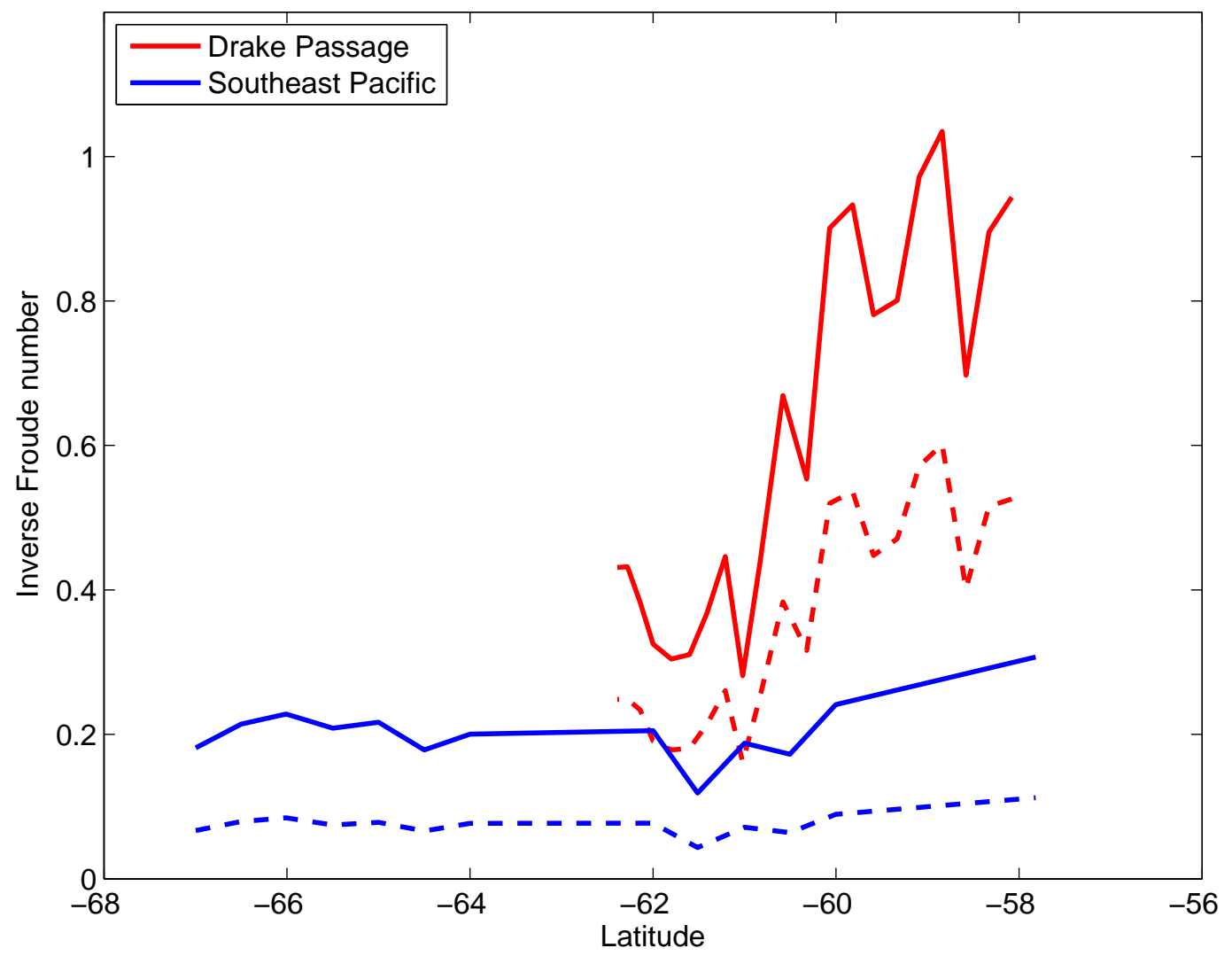

Figure 3-13: Inverse Froude number estimates for the Drake Passage (red) and the Southeast Pacific (blue) regions estimated using the lower (dashed) and the upper (solid) limits of effective topography estimate. 


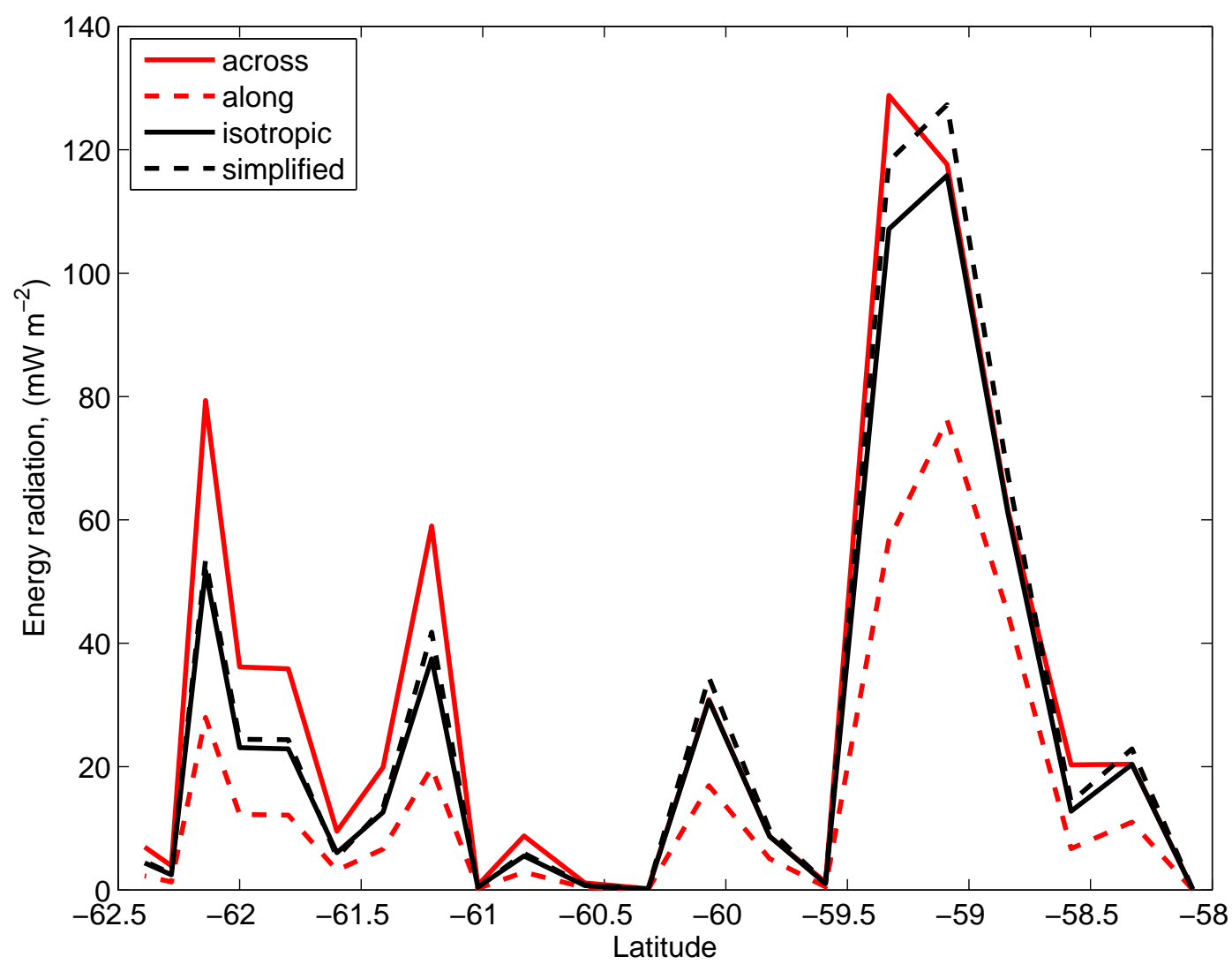

Figure 3-14: Energy radiation estimate $\left(\mathrm{mW} \mathrm{m}^{-2}\right)$ along the ALBATROSS section in the Drake Passage region. 


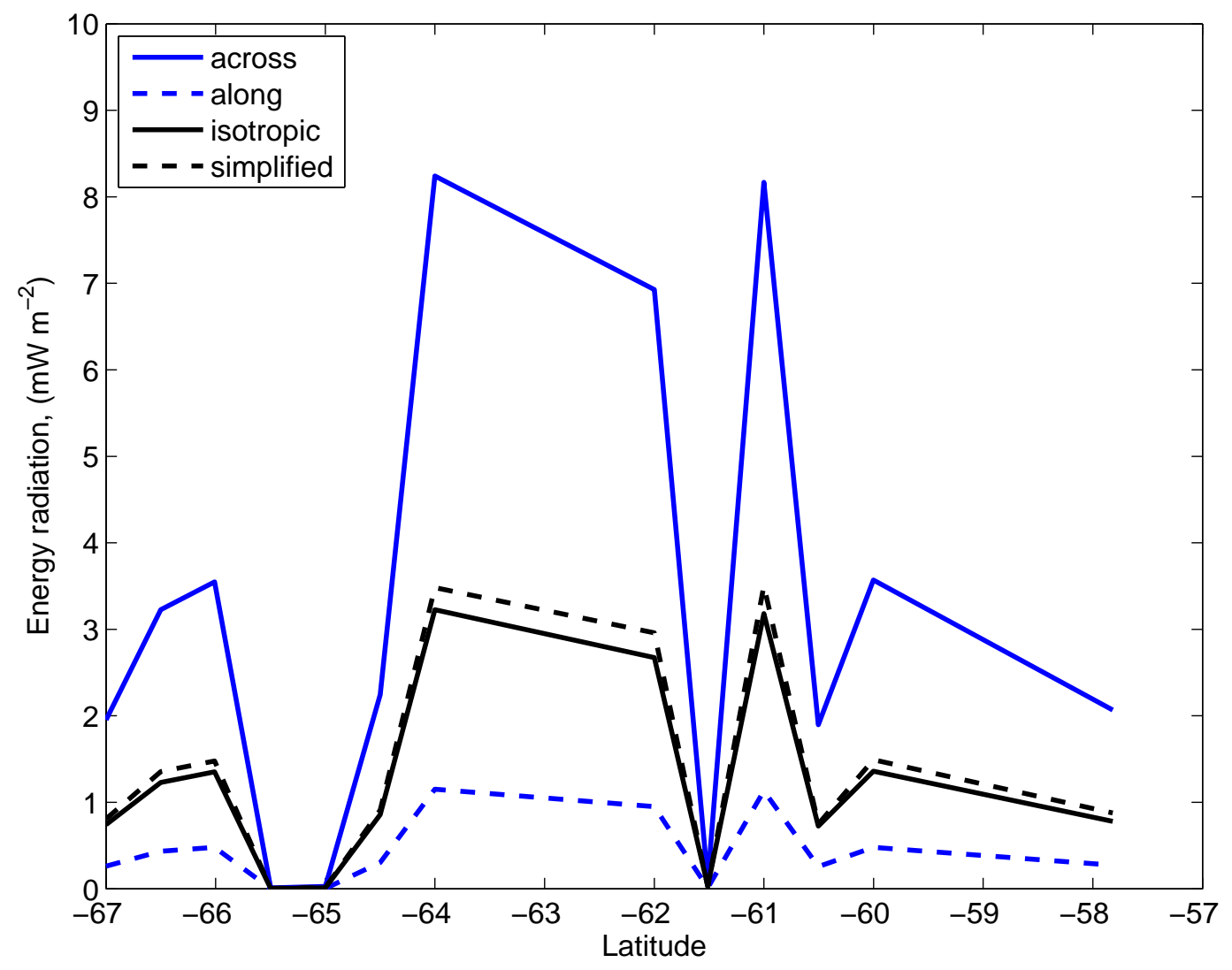

Figure 3-15: Energy radiation estimate $\left(\mathrm{mW} \mathrm{m}^{-2}\right)$ along the $\mathrm{P} 18$ section in the Southeast Pacific region. 


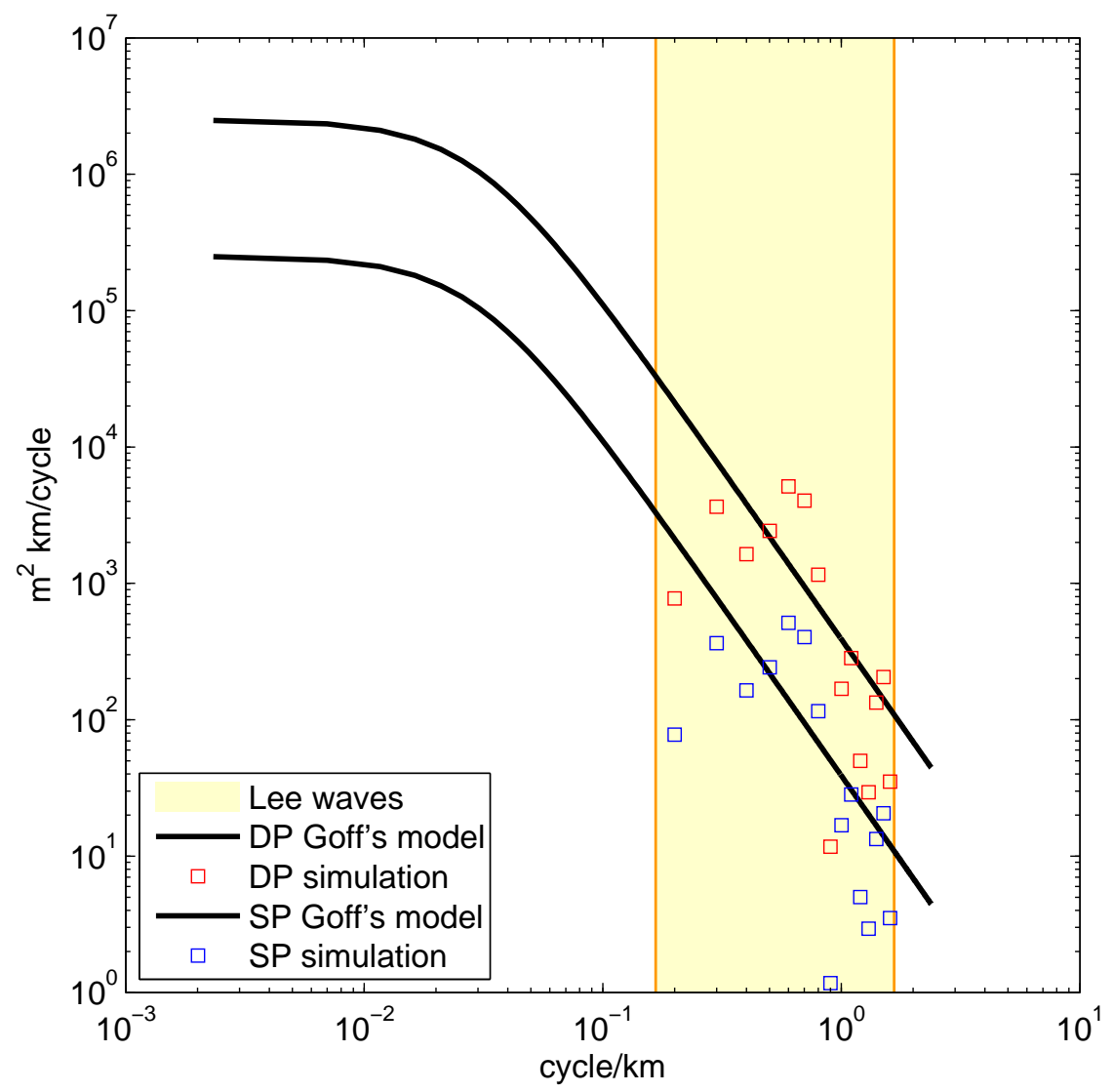

Figure 3-16: Bottom topography spectra used for simulations characteristic of the Drake Passage (red squares) and in the Southeast Pacific (blue squares) regions; corresponding Goff's model spectra are shown as black curves. 


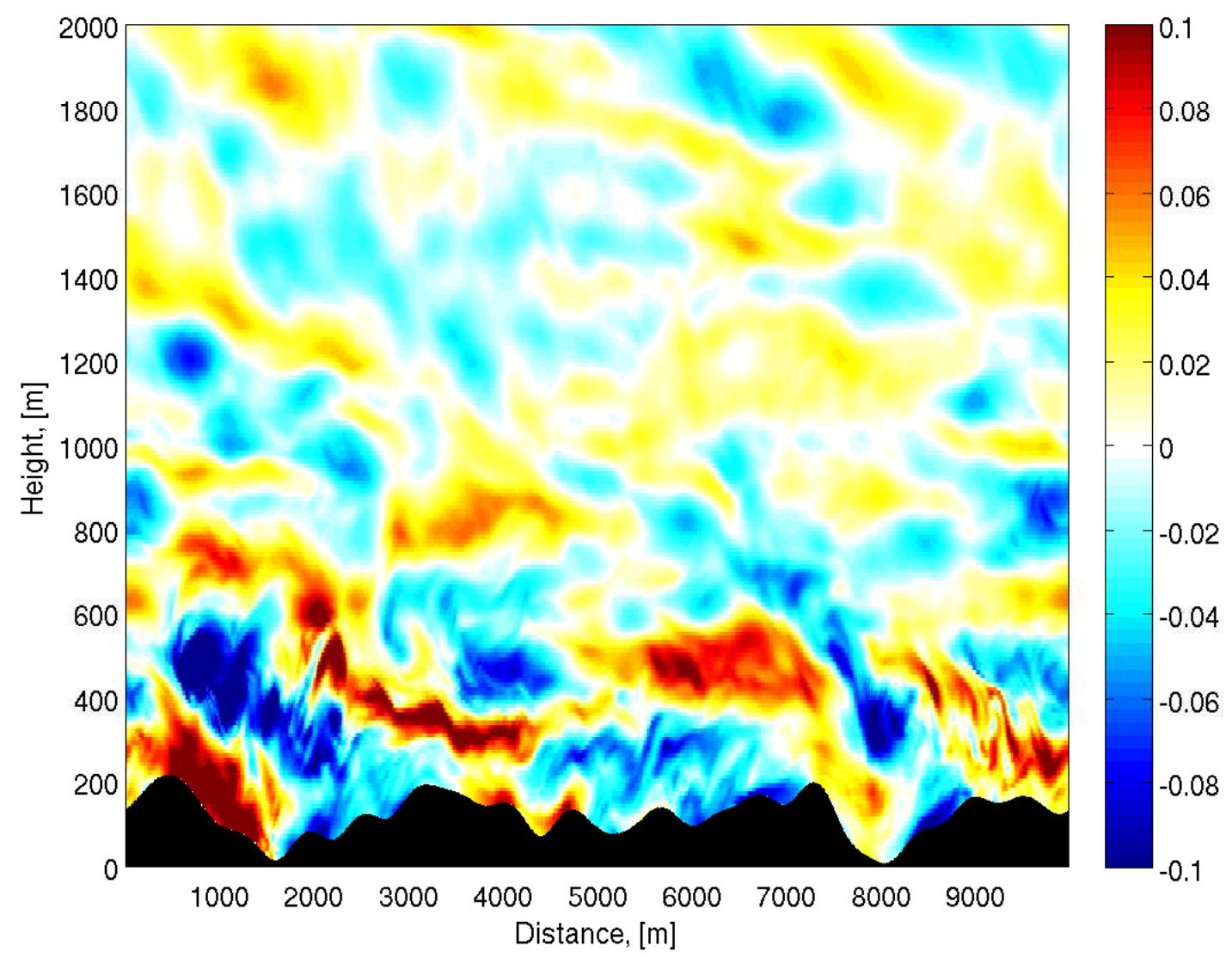

Figure 3-17: Snapshot of the wave zonal velocity $\left(\mathrm{m} \mathrm{s}^{-1}\right)$ from the Drake Passage simulation. 




Figure 3-18: Snapshot of the wave zonal velocity $\left(\mathrm{m} \mathrm{s}^{-1}\right)$ from the Southeast Pacific simulation. 


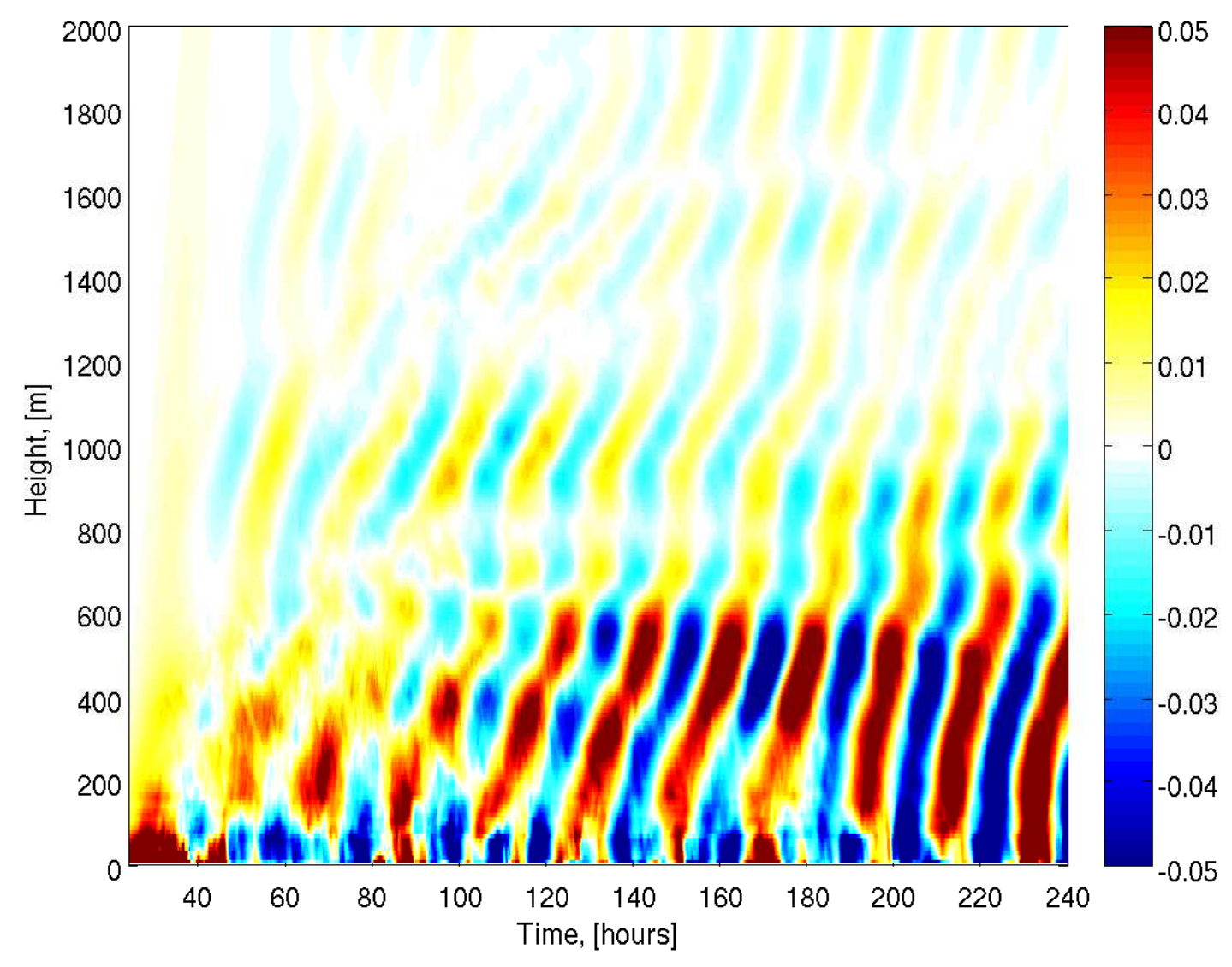

Figure 3-19: Time evolution of the zonally averaged meridional velocity component $\left(\mathrm{m} \mathrm{s}^{-1}\right)$ from the Drake Passage simulation. 


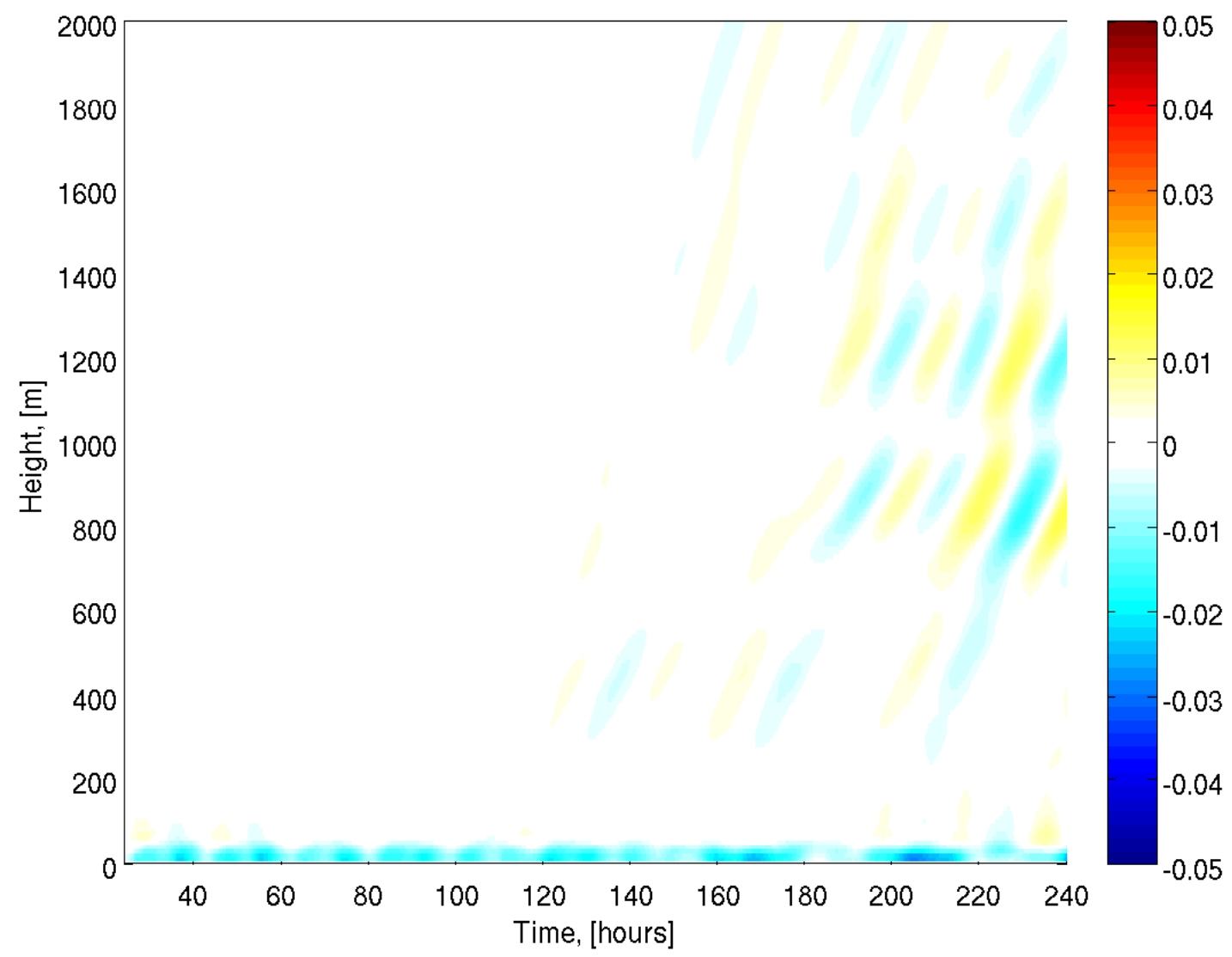

Figure 3-20: Time evolution of the zonally averaged meridional velocity component $\left(\mathrm{m} \mathrm{s}^{-1}\right)$ from the Southeast Pacific simulation. 


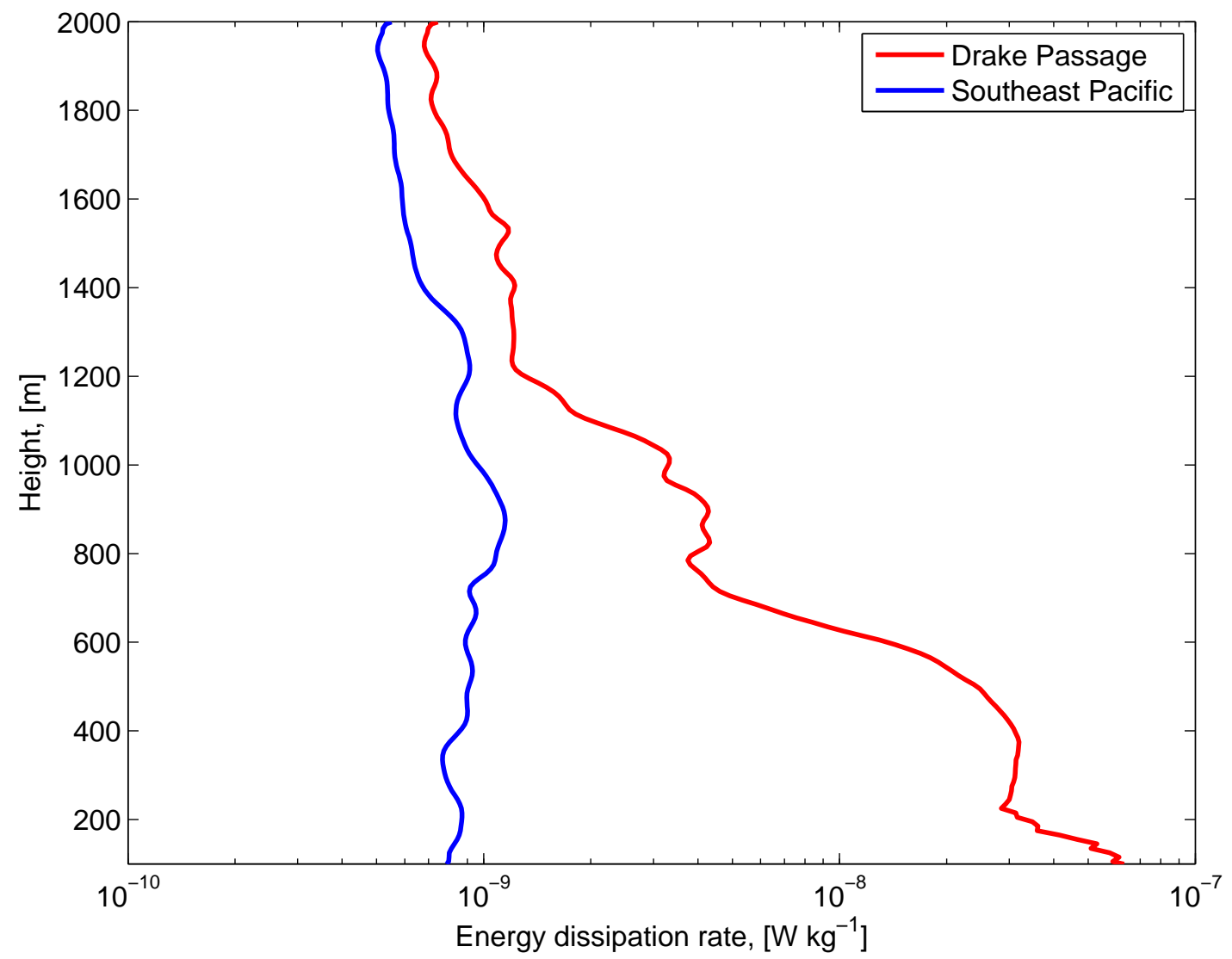

Figure 3-21: Profiles of energy dissipation rate $\left(\mathrm{W} \mathrm{kg}^{-1}\right)$ diagnosed from the Drake Passage (red) and the Southeast Pacific (blue) simulations. 


\section{Chapter 4}

\section{3-D Theory and Simulations}

\subsection{Introduction}

In the previous chapters we showed that radiation of internal waves by mean geostrophic flows depends mostly on topographic scales from about $600 \mathrm{~m}$ to $6 \mathrm{~km}$ for parameters

characteristic of the Southern Ocean. At these scales, ocean bottom topography is characterized by abyssal hills with characteristic scales of less than $10 \mathrm{~km}$ which cover approximately $80 \%$ of the world's seafloor. Abyssal hills are topographic features created by vulcanism and block faulting at the ridge crests. They are weakly anisotropic being elongated perpendicular to the direction of spreading (Goff and Jordan, 1988). The goal of this chapter is to extend the 2-D theory of radiation of internal waves from topography developed in Chapter 2 to the full complexity of 3-D topography.

The nature of mean flow-topography interaction is different in 2-D and 3-D. In $2-\mathrm{D}$, the mean flow is forced to go over topography and radiate internal waves until it becomes blocked at high inverse Froude numbers and the radiation saturates. In 3-D, however, at finite inverse Froude numbers the mean flow can partially split and go around topography rather than over it, and potentially reduce wave radiation.

Idealized 2-D numerical simulations show that wave energy dissipation within the bottom several hundred meters depends heavily on the vertical shear associated with inertial oscillations. Bottom intensified inertial oscillations are driven by the divergence of internal wave momentum fluxes. We wish to investigate whether wave 
breaking and the subsequent wave-mean flow resonant feedback carry over to 3-D, despite the expected changes in the scale and amplitude of generated waves.

In order to test whether the results described in Chapter 2 can be applied to the 3 -D wave radiation problem, we extend both linear theory and numerical simulations to a 3-D flow over a 2-D bottom topography. In section 2, we present 3-D linear wave generation theory and discuss how it compares to 2-D theory. In section 3 , we describe the numerical simulation setup. In section 4, we compare the predictions of 3-D linear theory, with results from numerical simulations. Finally, results are summarized in section 5 .

\subsection{Theory}

We extend the 2-D internal wave generation theory described in section 2.4.3 to a 3-D, $(x, y, z)$, domain with arbitrary bottom topography $h=h(x, y)$. The zonally averaged flow is assumed to be the same as in the 2-D problem described before, i.e the sum of a mean geostrophic flow and a spatially uniform inertial oscillation,

$$
\begin{aligned}
& \bar{u}(t)=U_{G}+U_{I} \cos f\left(t-t_{0}\right), \\
& \bar{v}(t)=-U_{I} \sin f\left(t-t_{0}\right),
\end{aligned}
$$

The dynamics of internal waves is governed by the set of 3-D equations (2.15) through (2.18) with the lower boundary condition (2.19). To simplify the analysis, as in 2-D theory, we use Rayleigh damping to represent wave dissipation, as per eq. (2.26).

In order to compute wave radiation, it is convenient to change reference frame to the coordinate system $(\xi, \eta)$ moving with the time dependent mean flow $\overline{\mathbf{u}}(t)=$ $(\bar{u}(t), \bar{v}(t))$,

$$
\xi=x-\int_{t_{0}}^{t} \bar{u}(t) d t, \quad \eta=y-\int_{t_{0}}^{t} \bar{v}(t) d t
$$

In the moving reference frame, the dynamics in (2.15) through (2.18) can be reduced 
to a single equation for the vertical velocity $w^{(1)}$,

$$
\left(\partial_{t t}+2 \lambda \partial_{t}+\lambda^{2}\right) \nabla^{2} w^{(1)}+N^{2} \nabla_{H}^{2} w^{(1)}+f^{2} w_{z z}^{(1)}=0
$$

where $\nabla^{2}=\partial_{\xi \xi}+\partial_{\eta \eta}+\partial_{z z}$ and $\nabla_{H}^{2}=\partial_{\xi \xi}+\partial_{\eta \eta}$ are the 3-D and the 2-D Laplace operators respectively. Top and bottom boundary conditions are of vanishing vertical velocity as $z \rightarrow \infty$ and zero velocity normal to topography,

$$
\left.w^{(1)}\right|_{z=0}=\overline{\mathbf{u}} \cdot \nabla_{H} h
$$

Periodic topography implies a periodic solution, and we can expand the solution into Fourier modes in the $(\xi, \eta)$-coordinate frame. The bottom boundary condition takes the form,

$$
\left.\tilde{w}^{(1)}\right|_{z=0}=\hat{h} \frac{\partial}{\partial t} e^{i \int_{t_{0}}^{t}(\mathbf{k} \cdot \overline{\mathbf{u}}) d t}
$$

where $\tilde{w}^{(1)}$ is the Fourier transform of the wave vertical velocity in the moving $(\xi, \eta)$ coordinate frame and $\hat{h}$ is the Fourier transform of the bottom topography in the fixed $(x, y)$-coordinate frame. Using the mean flow in (4.1) and (4.2) and applying the Jacobi-Anger expansion, the bottom boundary condition can be written as a superposition of plane waves,

$$
\left.\tilde{w}^{(1)}\right|_{z=0}=i \hat{h} \sum_{m=-\infty}^{\infty} \sum_{n=-\infty}^{\infty} \sigma_{m n} J_{m}\left(\beta_{k}\right) J_{n}\left(\beta_{l}\right) e^{i\left[\sigma_{m n}\left(t-t_{0}\right)+\frac{\pi}{2} n\right]},
$$

where $\sigma_{m n}=(m+n) f+U_{G} k$ is the intrinsic frequency of the wave harmonic $(m, n)$, i.e. the $(m+n)$-th harmonic of inertial frequency $f$, Doppler shifted by the constant zonal geostrophic flow $U_{G}$. The parameters $\beta_{k}=U_{I} k / f$ and $\beta_{l}=U_{I} l / f$ are the zonal and meridional maximum excursions of a water parcel advected by an inertial oscillation of amplitude $U_{I}$, and $J_{m}$ and $J_{n}$ are Bessel functions of the first kind.

The bottom boundary condition (4.7) is analogous to the 2-D bottom boundary condition (2.29). It shows that a superposition of a constant mean flow and an inertial oscillation over bottom topography results in radiation of a set of waves 
which are harmonics of the inertial frequency. The main difference is that, in $3-\mathrm{D}$, the topography can vary in both zonal and meridional directions, and hence there are two independent harmonic numbers $m$ and $n$. The amplitude of each harmonic is controlled by the excursion parameters $\beta_{k}$ and $\beta_{l}$ which can be different depending on the aspect ratio of topography. The phase shift $\frac{\pi}{2} n$ results from the phase shift between the zonal and meridional components of the prescribed inertial oscillation in (2.23) and (2.24). In the absence of topography variations in meridional direction, $l \rightarrow 0$, the bottom boundary condition (4.7) reduces to its $2-\mathrm{D}$ equivalent (2.29).

Solutions for $\tilde{w}^{(1)}$ are obtained by projecting the equation (4.4) onto Fourier modes, and imposing the bottom boundary condition (4.7). The solution is a superposition of modes,

$$
\tilde{w}^{(1)}=\sum_{m=-\infty}^{\infty} \sum_{n=-\infty}^{\infty} \tilde{w}_{m n}^{(1)}
$$

each of which takes the form,

$$
\tilde{w}_{m n}^{(1)}=i \hat{h} \sigma_{m n} J_{m}\left(\beta_{k}\right) J_{n}\left(\beta_{l}\right) e^{i \theta_{m n}}, \quad \theta_{m n}=\mu_{m n} z+\sigma_{m n}\left(t-t_{0}\right)+\frac{\pi}{2} n .
$$

The coefficient $\mu_{m n}$ is a complex number whose real and imaginary parts represent, respectively, the wave vertical wavenumber and an inverse decay scale due to damping,

$$
\mu_{m n}^{2}=\left(k^{2}+l^{2}\right) \frac{N^{2}-\left(\sigma_{m n}-i \lambda\right)^{2}}{\left(\sigma_{m n}-i \lambda\right)^{2}-f^{2}} .
$$

The pressure modes associated with $\tilde{w}_{m n}^{(1)}$ can be obtained by transforming $(2.15)$ through (2.18) into the moving reference,

$$
\tilde{p}_{m n}^{(1)}=\frac{i \hat{h} \sigma_{m n}^{2}}{\sqrt{k^{2}+l^{2}}} \frac{\mu_{m n}}{\sqrt{k^{2}+l^{2}}} \frac{\left(\sigma_{m n}-i \lambda\right)^{2}-f^{2}}{\sigma_{m n}\left(\sigma_{m n}-i \lambda\right)} J_{m}\left(\beta_{k}\right) J_{n}\left(\beta_{l}\right) e^{i \theta_{m n}} .
$$

The $\tilde{w}^{(1)}$ and $\tilde{p}^{(1)}$ solutions in (4.9) and (4.11) can then be used to compute the bottom value of energy radiation averaged over the horizontal plane and over an 
inertial period,

$$
\begin{gathered}
\overline{w^{(1)} p^{(1)}}=\frac{1}{4 \pi^{2}} \int_{-\infty}^{\infty} \int_{-\infty}^{\infty} \frac{\mathcal{P}(\mathbf{k})}{\sqrt{k^{2}+l^{2}}} \sum_{m=-\infty}^{\infty} \sum_{n=-\infty}^{\infty} \sum_{p=-\infty}^{\infty} \sum_{q=-\infty}^{\infty} \sigma_{m n}^{2} \sigma_{p q} \times \\
\times J_{m}\left(\beta_{k}\right) J_{n}\left(\beta_{l}\right) J_{p}\left(\beta_{k}\right) J_{q}\left(\beta_{l}\right) \Re\left(\frac{\mu_{m n}}{\sqrt{k^{2}+l^{2}}} \frac{\left(\sigma_{m n}-i \lambda\right)^{2}-f^{2}}{\sigma_{m n}\left(\sigma_{m n}-i \lambda\right)} e^{i \frac{\pi}{2}(n-q)}\right) d \mathbf{k},
\end{gathered}
$$

where $\mathcal{P}(\mathbf{k})$ is the spectrum of topography.

The energy radiation averaged in space and time is given by the sum over all possible couples of harmonics $(m, n)$ and $(p, q)$ whose frequencies $\sigma_{m n}=\sigma_{p q}$, i.e. $n+m=p+q$. Other combinations of two harmonics generate no energy flux averaged over an inertial period. The expression in (4.12) reduces to the 2-D energy radiation expression in (2.35) in the limit of $l \rightarrow 0$ and for a monochromatic topography. Also (4.12) reduces to the well known expression for lee wave radiation in the limit of a zero inertial oscillation, i.e. $U_{I}=0$ (Bell, 1975a,b).

\subsection{Experiment setup}

We use the nonhydrostatic configuration of the MIT general circulation model (Marshall et al., 1997). The numerical simulation setup is similar to that used in the idealized two-dimensional simulations described in Chapter 2. Here, we increase the model grid to a 3-D, doubly periodic domain with $L_{x} \times L_{y} \times H_{z}=2 \mathrm{~km} \times 2 \mathrm{~km} \times$ $7 \mathrm{~km}$. We use a resolution of $\Delta x=16.6 \mathrm{~m}$ in the horizontal and $\Delta z=10 \mathrm{~m}$ in the vertical which gradually stretches to $\Delta z=300 \mathrm{~m}$ in the region from 2 to $7 \mathrm{~km}$ above the bottom. The bottom topography used in the simulations is shown in Fig. 4-1 and given by,

$$
h(x, y)=h_{T} \cos \left(k_{T} x\right) \cos \left(l_{T} y\right)
$$

where $h_{T}$ is the amplitude of topography, and $k_{T}, l_{T}$ are its zonal and meridional wavenumbers. The topographic wavenumbers $k_{T}$ and $l_{T}$ do not vary between the simulations and are set to $k_{T}=l_{T}=2 \pi / 2 \mathrm{~km}^{-1}$.

There is a sponge layer between 2 and $7 \mathrm{~km}$ above the bottom to absorb waves that 
do not break in the bottom kilometer. At the bottom, we use free-slip boundary conditions. A depth-independent, horizontally uniform zonal mean flow $U_{G}=0.1 \mathrm{~m} \mathrm{~s}^{-1}$ is forced by adding a body force $f U_{G}$ to the meridional momentum equation, representing a barotropic pressure gradient in geostrophic balance with $U_{G}$ at all depths (see Chapter 2 for a discussion of these choices). Because of the large computational cost of 3-D simulations, we run only three different experiments with topography amplitudes $h_{T}=40,60$, and $80 \mathrm{~m}$ which span the range of topographic amplitudes where time-dependent waves are radiated according to the 2-D simulations. All simulations are run for 10 days, which is sufficient to reach a statistically steady state.

\subsection{Results}

In this section we test the predictions of linear theory versus numerical simulations and compare the results to the 2-D problem discussed in Chapter 2. As before, we decompose the model solution into a spatial mean (the mean) and deviations from the mean (the waves). We demonstrate that in 3-D, as in 2-D, at finite inverse Froude numbers the radiation of waves results in bottom intensified inertial oscillations which favor local wave breaking.

\subsubsection{Inverse Froude number}

In order to compare the results of the $2-\mathrm{D}$ and $3-\mathrm{D}$ wave radiation problems, we need to use consistently defined inverse Froude numbers. In 2-D we used inverse Froude number defined as,

$$
F r_{2 D}^{-1}=\frac{N h_{T}}{U_{G}}
$$

where $N$ is the bottom stratification, $U_{G}$ is the bottom geostrophic velocity, and $h_{T}$ is the amplitude of topography in (2.25). This form of the inverse Froude number follows from a more general definition for the multichromatic topography (3.17) applied to 
the spectrum of (2.25) given by,

$$
\mathcal{P}_{2 D}(\mathbf{k})=\pi^{2} h_{T}^{2} \cdot\left[\delta\left(k-k_{T}\right)+\delta\left(k+k_{T}\right)\right] \cdot \delta(l),
$$

where spectrum $\mathcal{P}_{2 D}(\mathbf{k})$ is normalized according to (3.2).

Equivalently, applying the same definition to the spectrum of topography (4.13) used in the 3-D simulations and given by,

$$
\mathcal{P}_{3 D}(\mathbf{k})=\frac{1}{4} \pi^{2} h_{T}^{2} \cdot\left[\delta\left(k-k_{T}\right)+\delta\left(k+k_{T}\right)\right] \cdot\left[\delta\left(l-l_{T}\right)+\delta\left(l+l_{T}\right)\right]
$$

we obtain,

$$
F r_{3 D}^{-1}=\left(\frac{1}{2} \frac{k_{T}}{\sqrt{k_{T}^{2}+l_{T}^{2}}}\right)^{1 / 2} \frac{N h_{T}}{U_{G}}
$$

In the special case of $k_{T}=l_{T}$, considered here, we get,

$$
F r_{3 D}^{-1}=\frac{1}{2^{3 / 4}} \frac{N h_{T}}{U_{G}}
$$

The prefactor $2^{-3 / 4}$ in (4.17) accounts for the different fractions of area occupied by topography in $3-\mathrm{D}$ versus $2-\mathrm{D}$ (infinite meridional ridges in $2-\mathrm{D}$ and axisymetric bumps in 3-D).

\subsubsection{Inertial oscillations}

The time evolution of the zonally averaged velocity for the simulation with $h_{T}=60 \mathrm{~m}$, corresponding to $\mathrm{Fr}^{-1}=0.36$, is shown in Fig. 4-2. It shows strong and coherent inertial oscillations which are not initially prescribed or externally forced in the simulations. They develop spontaneously at the bottom and reach finite amplitude within a few days. These oscillations are depth dependent with a vertical scale of about $1 \mathrm{~km}$ and are significantly intensified in the bottom 500-600 m.

The magnitude of the inertial oscillations at the seafloor as a function of time is shown in Fig. 4-3 for simulations corresponding to $F r^{-1}=0.24,0.36$, and 0.48 . The amplitude of inertial oscillations grows from less than $0.01 \mathrm{~m} \mathrm{~s}^{-1}$ (created supposedly 
by transient wave effect on the mean flow) to about $0.07-0.08 \mathrm{~m} \mathrm{~s}^{-1}$ within $3-4$ days of simulation. The equilibrated value of inertial oscillations is independent of topography amplitude and it is $30 \%$ smaller in $3-\mathrm{D}$ than in $2-\mathrm{D}$ simulations.

We have not extended the wave-mean flow feedback theory to 3-D waves due to the algebraic complexity of the problem. However a few points are clear. The spontaneous emergence and the growth rates of inertial oscillations are very similar in 2-D and 3-D simulations, strongly supporting the inference that the 2-D resonant feedback mechanism described in Chapter 2, section 2.4.4, operates also in 3-D. The inertial oscillations are driven by the wave momentum fluxes, whose amplitude depends on the Bessel function of the excursion parameter, $U_{I} k / f$, and they equilibrate when those fluxes vanish. Although an exact value of equilibrated amplitude depends on all components of the wave momentum flux, we can find its order of magnitude using flux formed by the first two harmonics. This flux vanishes at the first zero of the Bessel function $J_{0}$, when its argument is equal to $\sim 2.4$, implying that the equilibrated amplitude is given by $U_{I} \approx 2.4 f / k \approx 0.08 \mathrm{~m} \mathrm{~s}^{-1}$.

\subsubsection{Wave radiation}

Linear theory is now tested against 3-D simulations and compared to the equivalent 2-D results. We diagnose the energy flux from simulations using deviations from the zonal mean in pressure and vertical velocity and averaging it spatially in both zonal and meridional directions and temporally over several inertial periods. The energy flux is then compared to the 3 -D prediction in (4.12) with the monochromatic spectrum in (4.16) and the 2-D prediction (2.35) corresponding to the monochromatic spectrum in (4.15).

The decay of wave energy flux with height above the bottom is shown in Fig. 4-4 for all three simulations. In general, the vertical structure of wave fluxes is similar to the corresponding 2-D simulations. The magnitude of the energy flux increases with topography amplitude. As waves radiate away from topography, the energy flux drops substantially within the bottom $500 \mathrm{~m}$ where waves break and dissipate.

Fig. 4-5 shows the bottom values of the energy flux from 2-D and 3-D numerical 
simulations compared to the corresponding linear theory predictions. Both the 2-D and 3-D energy radiation estimates scale linearly with $\mathrm{Fr}^{-2}$ until radiation saturates. While 2-D radiation saturates at $F r_{c}^{-1} \approx 0.7,3$-D radiation seems to saturate at lower inverse Froude number, possibly as small as $F r_{c}^{-1} \approx 0.5$, supposedly due to both topographic blocking and mean flow splitting effects. However, the three simulations that we run are not sufficient to unequivocally determine the saturation $F r_{c}^{-1}$.

The 2-D and 3-D linear lee wave radiation estimates are collapsed into a single line when geometrical differences are included in the inverse Froude number definition (4.17). This result is consistent with the discussion in Chapter 3 about the reduction of wave radiation in $3-\mathrm{D}$ compared to $2-\mathrm{D}$ as a result of the fact that the mean flow sees a reduced effective topography in 3-D. Furthermore the estimated radiation from numerical simulations are very close to the predictions of linear theory, suggesting that the theoretical framework used in Chapter 2 and in this chapter are very relevant for the parameter range considered.

At equilibrium inertial oscillations have magnitudes of $0.12 \mathrm{~m} \mathrm{~s}^{-1}$ and $0.08 \mathrm{~m} \mathrm{~s}^{-1}$ in the 2-D and 3-D simulations respectively and increase the energy radiation by about $30 \%$ compared to the prediction of linear lee wave theory with no inertial oscillations. The effect of inertial oscillations on the wave generation is well captured by the linear theory of radiation of inertial frequency harmonics imposing the amplitudes of inertial oscillations as diagnosed from the simulations.

The possible saturation of wave radiation at $F r_{c}^{-1}=0.5$ for the 3 -D case, lower than in the 2-D simulations, would have an implication for the energy radiation estimates presented in Chapter 3. If $\mathrm{Fr}_{c}^{-1}=0.5$ is used instead of 0.7 to limit wave radiation then the radiation estimate will be reduced from $15-31 \mathrm{~mW} \mathrm{~m}^{-2}$ to 13 $22 \mathrm{~mW} \mathrm{~m}^{-2}$ for the Drake Passage region, and remains the same for the Southeast Pacific.

\subsubsection{Wave breaking and dissipation}

As internal waves radiate away from topography they propagate through a strong inertial shear and their vertical wavenumber is squeezed until breaking occurs. Sim- 
ulations show that the bottom several hundred meters are characterized by vigorous turbulence, resulting from enhanced wave breaking and dissipation. Wave breaking is well illustrated in Fig. 4-6 which shows a snapshot of an isopycnal surface roughly $100 \mathrm{~m}$ above the bottom from the $h_{T}=60 \mathrm{~m}$ simulation. The surface undergoes displacements of several tens of meters on the horizontal scales of the underlying topography. At the wave crest, there is a convectively unstable region indicating that this wave is about to overturn and break.

We diagnose the turbulent dissipation rate $\epsilon$ from the simulations. Vertical profiles of time averaged dissipation rate are shown in Fig. 4-7. The turbulent dissipation rates increase with inverse Froude number. They are enhanced by an order of magnitude in the bottom $1 \mathrm{~km}$ where most of the wave breaking occurs and then decay above. Values of dissipation rate integrated over the bottom $1 \mathrm{~km}$ layer are 5, 9 and $11 \mathrm{~mW} \mathrm{~m}^{-2}$, corresponding to simulations with topographic amplitude 40, 60, and $80 \mathrm{~m}$ respectively. As in the $2-\mathrm{D}$ simulations, energy dissipation in the bottom kilometer is roughly $30 \%$ of the bottom value of energy radiation. Energy dissipation of $11 \mathrm{~mW} \mathrm{~m}^{-2}$ diagnosed from the simulation compares well with $10 \mathrm{~mW} \mathrm{~m}^{-2}$ obtained from observations in the Drake Passage region (Naveira-Garabato et al., 2004).

\subsection{Conclusions}

Ocean bottom topography at scales of less than $10 \mathrm{~km}$ is characterized by abyssal hills which are essentially three-dimensional. Wave radiation from 3-D topographic features may differ from 2-D, depending on the value of inverse Froude number characterizing the mean flow - topography interaction. Using linear theory and numerical simulations we showed that, when the difference in geometry between the two problems is included in the definition of inverse Froude number, wave radiation estimates in the 2-D and 3-D problems are similar and well described by linear theory up to $F r_{c}^{-1}=0.5$. We have not studied $F r_{c}^{-1}>0.5$, but we suspect saturation might occur before the value of $\mathrm{Fr}_{c}^{-1}>0.7$ found in 2-D.

Idealized simulations show that similarities overcome a few quantitative differences 
between the 3-D and 2-D problems. In both cases there are vigorous, vertically sheared inertial oscillations in the bottom several hundred meters. These oscillations emerge spontaneously as a result of a wave-mean flow resonant feedback, grow and reach magnitudes comparable to the mean flow. Vertical shear associated with inertial oscillations promotes wave breaking and results in local dissipation of $30 \%$ of the radiated energy.

Although it is not completely certain, we speculate that energy radiation might already saturate at $\mathrm{Fr}_{c}^{-1}=0.5$, lower than value of 0.7 in the 2-D simulations, which might be due partly to topographic blocking as in the 2-D problem and partly to a mean flow splitting effect. As the inverse Froude number exceeds its critical value mean flow splits and goes around topography rather that over it, limiting further increase in the wave energy radiation. The saturation at lower inverse Froude number in 3 -D than in 2-D has an effect on energy radiation estimates presented in Chapter 3. Applying a lower critical inverse Froude number limit we estimate that energy radiation would reduce to $13-22 \mathrm{~mW} \mathrm{~m}^{-2}$ for the Drake Passage region and would remain the same for the Southeast Pacific.

One of the limitations of the 3-D simulations is the use of a free-slip boundary condition. Although 2-D simulations showed that a no-slip boundary condition results in about 20-30\% decrease in wave energy dissipation it is not clear whether the same applies to the 3-D problem. The effect of the boundary condition on the wave radiation and dissipation strongly depends on the low-level dynamics of mean flow-topography interaction which is different in the 2-D and 3-D problems. More study is needed to quantify the effect of the bottom boundary condition on the wave radiation in the $3-\mathrm{D}$ simulations. 


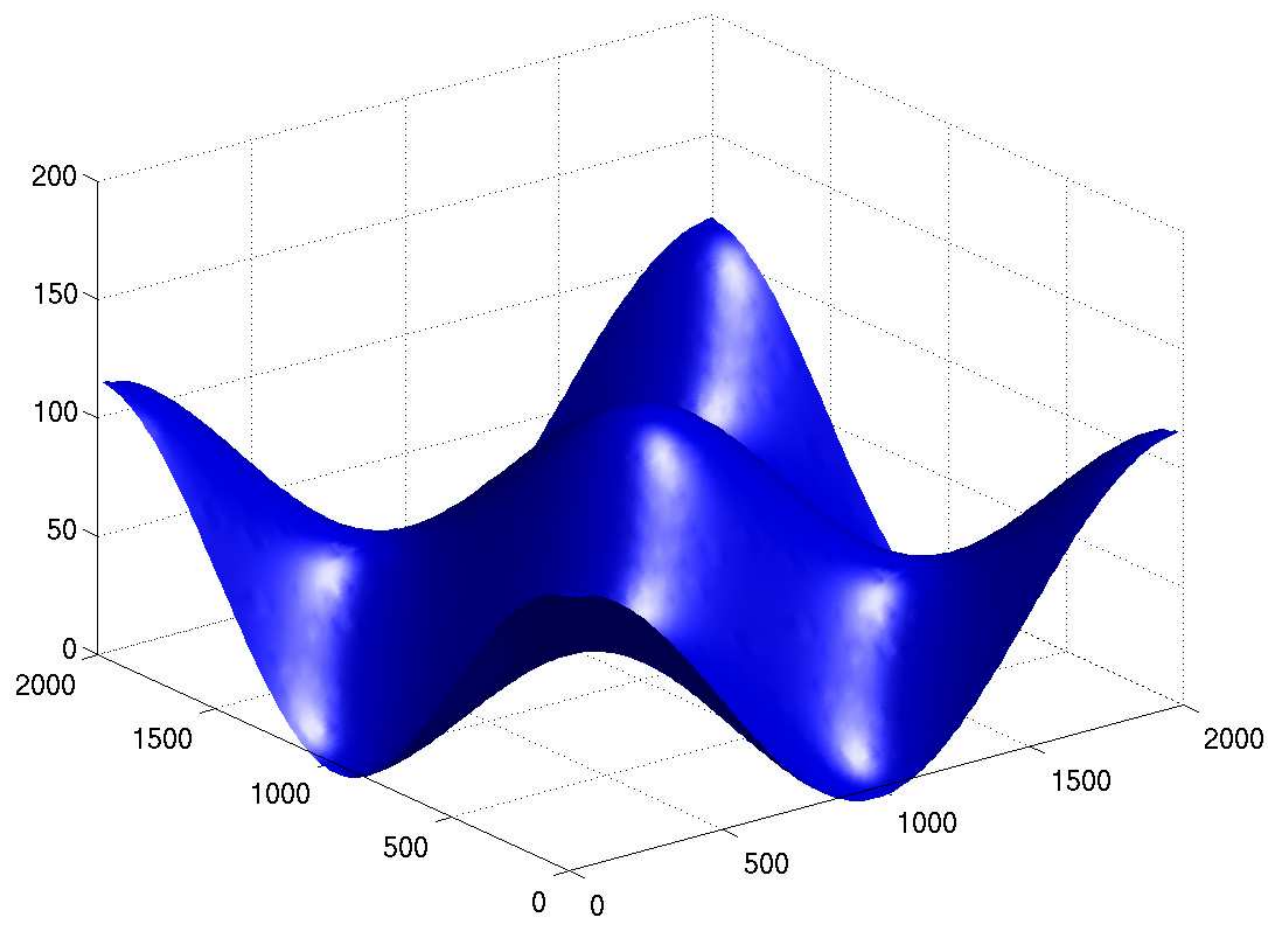

Figure 4-1: Bottom topography used in the 3-D simulations. Horizontal and vertical axes show distance in (m). 



Figure 4-2: Time evolution of zonal and meridional velocity components $\left(\mathrm{m} \mathrm{s}^{-1}\right)$ averaged in both zonal and meridional directions from $h_{T}=60 \mathrm{~m}$ simulation: (upper panel) zonal velocity component, a deviation from an externally prescribed $0.1 \mathrm{~m} \mathrm{~s}^{-1}$ zonal mean flow, (lower panel) meridional velocity component. 


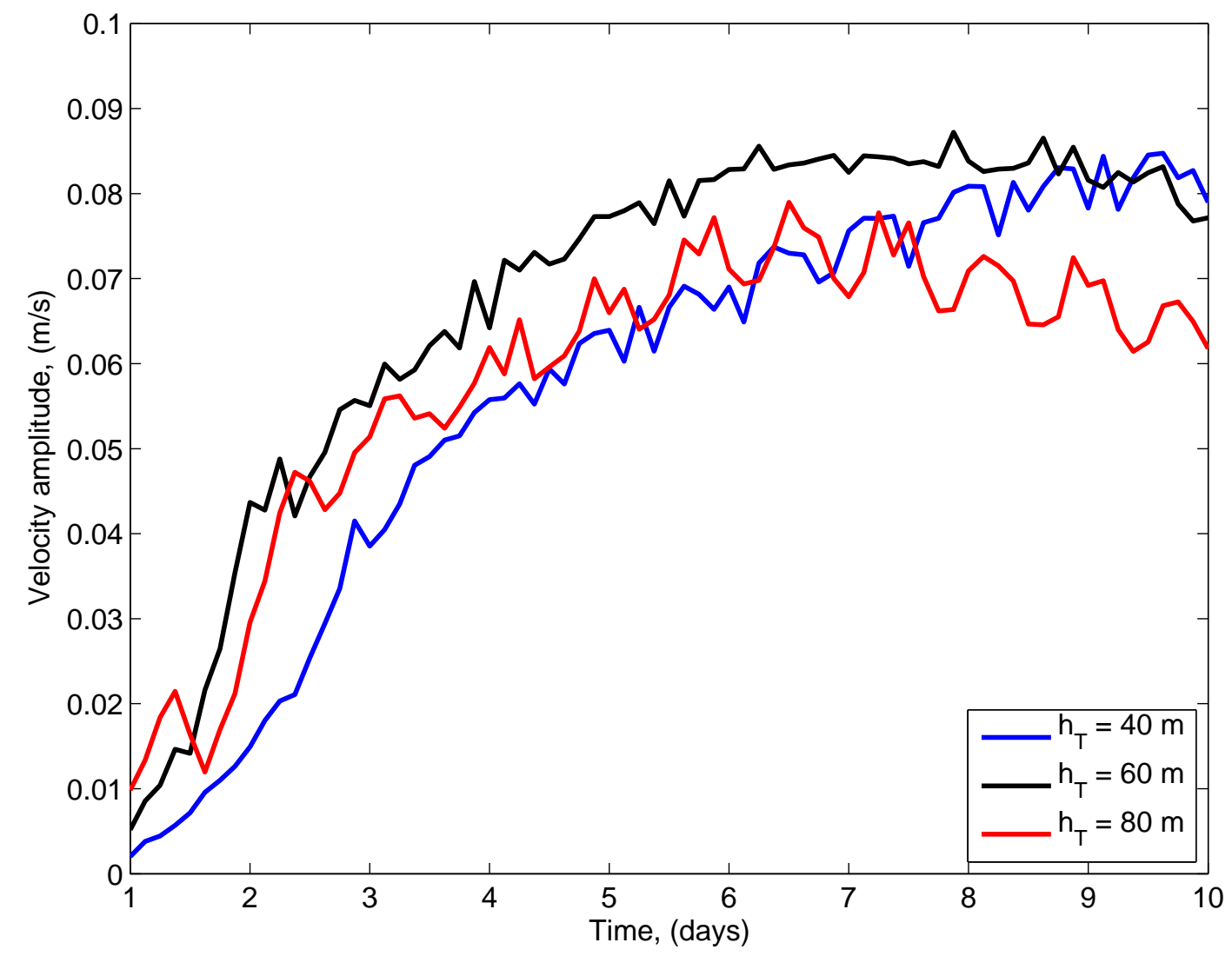

Figure 4-3: Evolution of the bottom value of inertial oscillation amplitude $\left(\mathrm{m} \mathrm{s}^{-1}\right)$ from different simulations. 




Figure 4-4: Profiles of the vertical energy flux $\left(\mathrm{mW} \mathrm{m}^{-2}\right)$ from different simulations. 


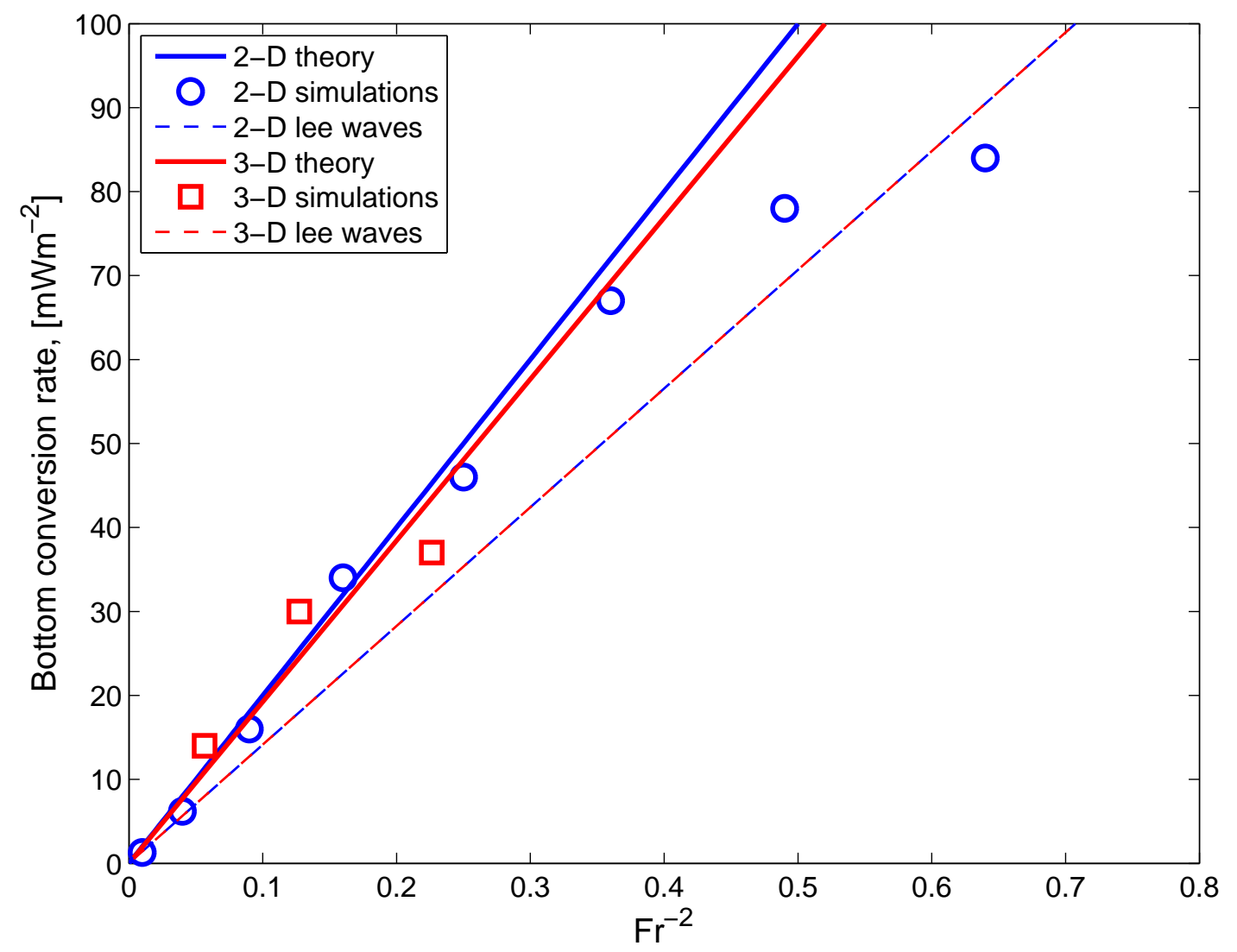

Figure 4-5: Energy conversion rate at the bottom as a function of $\mathrm{Fr}^{-2}$ : diagnosed from 2-D simulations (blue circles) and from 3-D simulations (red squares), predicted from linear lee wave theory (dashed lines), and predicted from linear theory for inertial harmonics (solid lines). 


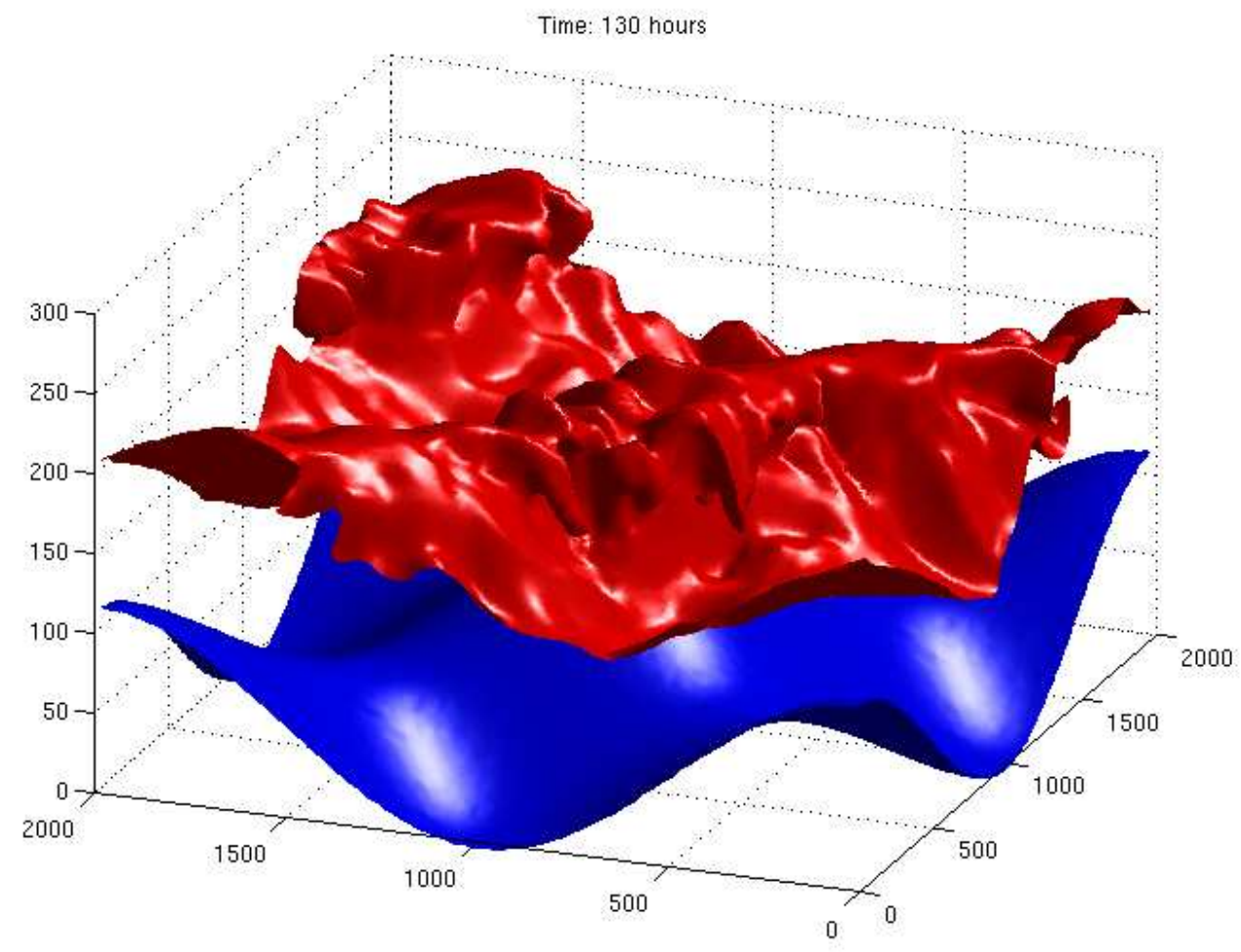

Figure 4-6: Snapshot of an isopycnal surface (red) roughly $100 \mathrm{~m}$ above topography from the $h_{T}=60 \mathrm{~m}$ simulation and underlying bottom topography (blue). 


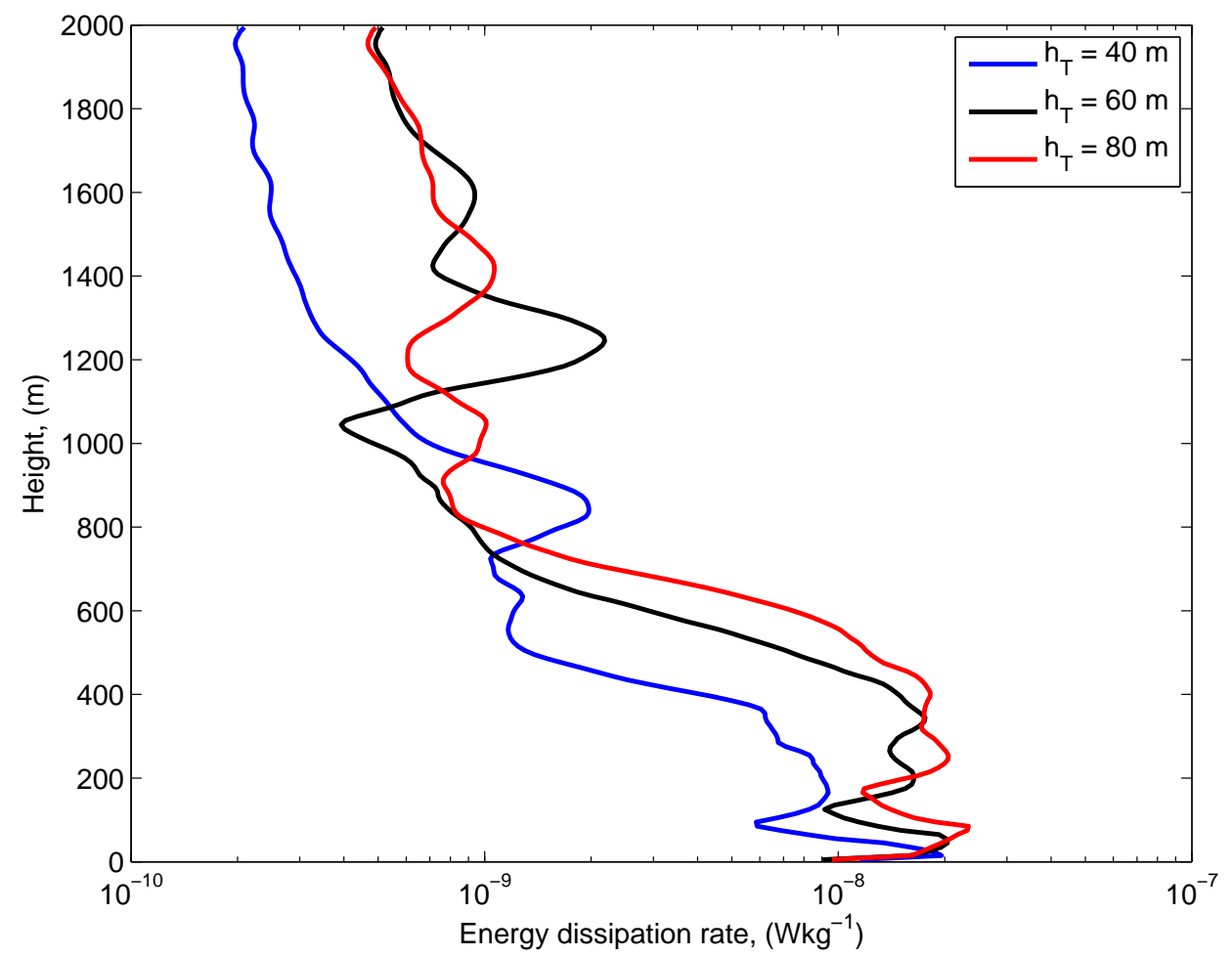

Figure 4-7: Vertical profiles of energy dissipation rate $\left(\mathrm{W} \mathrm{kg}^{-1}\right)$ from different simulation. 


\section{Chapter 5}

\section{Summary and Conclusions}

The central question of this thesis is whether internal waves generated by geostrophic flows interacting with small-scale bottom topography contribute significantly to turbulent mixing in the ocean interior. This work was motivated by recent observational estimates of enhanced mixing within $1 \mathrm{~km}$ of bottom topography in the Southern Ocean (Polzin and Firing, 1997; Naveira-Garabato et al., 2004; Kunze et al., 2006), a region where bottom geostrophic flows are very large. The analysis described in the thesis supports the inference that internal waves generated by geostrophic flows can drive enhanced abyssal mixing at rates inferred from observations. A summary of the novel results obtained in this thesis is presented first. Then the discussion shifts to the implications of this work for our understanding of the circulation of the Southern Ocean.

\subsection{Summary of the thesis}

In Chapter 2, the problem of wave radiation by geostrophic flows is addressed using 2-D linear theory and idealized numerical simulations. The analysis focuses on monochromatic topography and mean flow parameters characteristic of the Drake Passage region in the Southern Ocean. A major finding is that geostrophic flow - topography interaction results in a significant radiation of internal waves. Furthermore, and most importantly, a substantial fraction of the energy radiated into waves dissipates locally 
within a few hundred meters of topography as a result of wave breaking.

The analysis proceeds beyond the estimate of radiation and dissipation (a limit of previous oceanographic studies) and addresses the feedback of the waves on the large-scale flow. At finite inverse Froude numbers, internal waves drive inertial oscillations in the bottom several hundred meters through a resonant feedback mechanism. The inertial oscillations grow to become as large as the geostrophic flow and have a substantial amount of vertical shear on scales of a few hundred meters. This inertial shear modulates the upward propagating packets of short internal waves and promotes wave breaking. The resonant feedback mechanism is explored within a 2-D weakly nonlinear framework and confirmed with numerical simulations.

There are three different wave radiation regimes. At low inverse Froude numbers, lower than roughly 0.3 , wave radiation is characterized by quasi-stationary lee waves. Inertial oscillations grow slowly in time and do not develop enough over 10 days to significantly modify wave generation and radiation. At larger inverse Froude numbers, from 0.3 to 0.7 , inertial oscillations grow rapidly and reach amplitudes comparable to the mean flow within a few days, resulting in the radiation and breaking of time-dependent, multichromatic waves. At inverse Froude numbers greater than 0.7 some fraction of the mean flow becomes blocked by topography and energy radiation saturates, i.e. it does not increase with $\mathrm{Fr}^{-1}$.

The simulation results show that $30 \%$ of the radiated energy dissipates in the bottom kilometer. This result is consistent with observations that suggest a tight spatial correlation between mixing rates and toporgaphic roughness.

In Chapter 3, the results of linear theory and numerical simulations are used to estimate wave radiation from two regions of the Southern Ocean, the Southeast Pacific and Drake Passage, where bottom topography, velocity and stratification data are available. The regions represent a good testbed for the theory, because they are characterized respectively by low and high rates of abyssal mixing. Energy radiation by the geostrophic flows is estimated to be in the range $0.4-3.3 \mathrm{~mW} \mathrm{~m}^{-2}$ in the Southeast Pacific region and in the range $15-31 \mathrm{~mW} \mathrm{~m}^{-2}$ in the Drake Passage region. The uncertainty in these estimates is associated with changes in the orientation of 
the geostrophic flow with respect to topography: abyssal flows radiate more energy if they flow over topographic hills than along them. The difference in energy radiation between these two regions comes primarily from the difference in topographic roughness. The topographic spectral levels differ by an order of magnitude between the two regions, while the bottom velocity and stratification are comparable.

Two multichromatic topography simulations with topographic characteristics corresponding, respectively, to the Southeast Pacific and the Drake Passage regions are used to estimate the fraction of radiated wave energy that dissipates locally. The simulation representative of the Drake Passage region is characterized by strong, bottom intensified inertial oscillations, radiation of time-dependent waves, and enhanced wave breaking in the bottom several hundred meters. In the simulation representative of the Southeast Pacific region, on the other hand, waves are radiated in the form of linear lee waves without significant wave breaking and inertial oscillations above the bottom. Simulations confirm that a greater fraction of energy is dissipated in the time-dependent wave radiation regime. About $65 \%$ of the radiated energy is dissipated in the bottom kilometer in the Drake Passage and only $15 \%$ in the Southeast Pacific simulation.

To compare the energy radiation estimates with dissipation rates inferred from observations, first it is assumed that the geostrophic velocity spans all directions over an eddy turn-over time and, second, the results of numerical simulations are used to relate radiation to dissipation. The best estimate for local energy dissipation from linear theory and simulations is less than $1 \mathrm{~mW} \mathrm{~m}^{-2}$ in the Southeast Pacific and $O(10) \mathrm{mW} \mathrm{m}^{-2}$ in the Drake Passage. These estimates agree with observations and exceed background values of energy dissipation by 1 to 3 orders of magnitude.

In Chapter 4, 2-D wave radiation theory and numerical simulations are extended to 3-D topography. The analysis focuses on a periodic field of monochromatic 3-D bumps. With an appropriate definition of the inverse Froude number that accounts for geometrical differences in topography between the 2-D and 3-D problems (infinite meridional ridges in 2-D and axisymetric bumps in 3-D), wave radiation estimates are similar and well described by linear theory for up to $F r^{-1}=0.5$. Although the 
$\mathrm{Fr}^{-1}>0.5$ limit has not been fully studied, it appears as if saturation might occur before $\mathrm{Fr}^{-1} \approx 0.7$ (the value found in $2-\mathrm{D}$ ). The saturation of energy radiation at a lower inverse Froude number would imply a 30\% reduction of the Drake Passage estimate presented in Chapter 3, well within the uncertainty of the observations.

\subsection{Implications}

\subsubsection{Energetics and overturning circulation of the SO}

Enhanced dissipation rates maintained by geostrophic eddies might be a significant sink of the energy input by the wind into the general circulation of the ocean and an important driver of the lower cell of the Meridional Overturning Circulation. Wunsch (1998) estimated that the wind power input into large-scale geostrophic flows in the ocean is $\simeq 1 \mathrm{TW}$. The estimate has been confirmed by various authors in the following 10 years and the estimate hardly changes if one includes the work on the geostrophic eddy field (von Storch et al., 2007). The energy input is dominated by the work done in the Southern Ocean: 0.6 TW of the work is done south of $40^{\circ} \mathrm{S}$. This work powers the mean ACC system and is eventually converted into a vigorous geostrophic eddy field through baroclinic instability of the mean current.

If all the wind power input were to be converted into internal waves as geostrophic eddies rub over bottom topography and were subsequently dissipated through wave breaking, one should measure a uniform energy dissipation rate of $O(10) \mathrm{mW} \mathrm{m}^{-2}$ throughout the Southern Ocean (whose area has been taken to be $0.6 \times 10^{14} \mathrm{~m}^{2}$ ). Using Osborn's (1980) relationship between energy dissipation $\epsilon$ and turbulent diffusivity $\kappa$,

$$
\kappa=\Gamma \epsilon / N^{2}
$$

one obtains a diapycnal diffusivity of $O\left(2 \times 10^{-3}\right) \mathrm{m}^{2} \mathrm{~s}^{-1}$, using a typical bottom stratification of $N=10^{-3} \mathrm{~s}^{-1}$ and a mixing efficiency of $\Gamma=0.2$. Note that $\epsilon$ and $\kappa$ are expected to be enhanced only within $O(1) \mathrm{km}$ above bottom topography, where wave breaking occurs. Dissipation of $O(10) \mathrm{mW} \mathrm{m}^{-2}$ is only found in a few regions 
like Drake Passage characterized by rough topography (Naveira-Garabato et al., 2004; Kunze et al., 2006). In the Southeast Pacific, for example, characterized by smooth abyssal plains, energy dissipation estimate is an order of magnitude smaller.

Satellite bathymetry data (Smith and Sandwell, 1997) can be used to attempt an approximate estimate of the fraction of the Southern Ocean with topography that is as rough as in Drake Passage. The satellite data have a resolution of $15-20 \mathrm{~km}$ while wave radiation is linked to roughness at scales below $10 \mathrm{~km}$. Goff and Jordan (1988) show that small-scale roughness is proportional to roughness at scales of 10-100 km, which are well resolved by the altimetry. Topographic roughness at scales between $10-100 \mathrm{~km}$ is estimated as the rms height fluctuation in $1^{o} \times 1^{o}$ boxes (the height fluctuations are defined as departures from the best fit plane, in a least-square sense, in the $1^{\circ} \times 1^{\circ}$ box). The resulting map of roughness in the $45^{\circ} \mathrm{S}-65^{\circ} \mathrm{S}$ latitude band is shown in Fig. 5-2.

The above calculation shows that $15 \%$ of the Southern Ocean has topographic roughness in excess of $300 \mathrm{~m}$, the value representative for the Drake Passage region. While topographic roughness varies by an order of magnitude from place to place in the Southern Ocean, the wind power input (Wunsch, 1998) and EKE (Stammer, 1997) are quite uniform in the Southern Ocean (only a factor of 2 to 3 variability along the ACC). Hence, only in 15\% of the area of the Southern Ocean energy dissipation rates due to wave radiation are expected to match the local wind energy input. In the other $85 \%$, dissipation in the bottom boundary layer is a likely candidate to balance wind energy input, because there is not much evidence of energy radiation away from the Southern Ocean to other latitudes (Mazloff, 2008). Indeed, Sen et al. (2008) use a combination of near-bottom velocities from current meters and surface velocities from satellite altimetry and estimate that the global energy dissipation rate of geostrophic flows by quadratic bottom boundary layer drag is in the range of 0.2 to $0.8 \mathrm{TW}$, i.e it can easily account for $85 \%$ of $0.6 \mathrm{TW}$.

The $15 \%$ fraction of the wind energy input converted into internal wave radiation and dissipation has a significant impact on the MOC. If $15 \%$ of the Southern Ocean has rough topography to support wave radiation and breaking, the area- 
averaged energy dissipation rate and diapycnal diffusivity reduce to $O(1) \mathrm{mW} \mathrm{m}^{-2}$ and $O\left(2 \times 10^{-4}\right) \mathrm{m}^{2} \mathrm{~s}^{-1}$ respectively. These values are still an order of magnitude larger than the background values and can support a large cross-isopycnal circulation. If we assume that the depth integrated energy dissipation rate of $E=O(1) \mathrm{mW} \mathrm{m}^{-2}$ maintains a cross isopycnal flow by raising its potential energy in the bottom $H=1 \mathrm{~km}$ uniformly throughout the area of the Southern Ocean, $A=0.6 \times 10^{14} \mathrm{~m}^{2}$, then,

$$
\iint \Delta \rho g w d z d A \approx \int \Gamma E d A
$$

where integral on the left-hand side is the potential energy required to lift the abyssal, dense waters between isopycnals separated by $\Delta \rho, w$ is the vertical velocity, and $g$ is gravity. The integral on the right-hand side is the potential energy generated through wave breaking. Using this expression and parameters typical for the Southern Ocean, we find a cross-isopycnal transport of,

$$
\Psi=w A=\frac{\Gamma E A}{\rho_{0} N^{2} H^{2}} \approx O(10) S v
$$

Thus, the wind energy input converted into internal wave radiation and dissipation is sufficient to power up to $O(10) \mathrm{Sv}$ of overturning circulation in the bottom kilometer uniformly throughout the Southern Ocean. This estimate agrees with the transport of Antarctic Bottom Water (Ganachaud and Wunsch, 2000; Sloyan and Rintoul, 2001) and the rate of overturning circulation of the lower cell of the MOC (Ito and Marshall, 2008) which span the bottom kilometer of the Southern Ocean. That said, it should be clear that these estimates are very uncertain and more work and measurements are needed to better constrain the global budgets.

\subsubsection{Geostrophic flows and abyssal mixing in the global ocean}

The physics of wave radiation by geostrophic flows described in the thesis applies

to the global ocean. The Southern Ocean is special because bottom geostrophic 
velocities are very large and tides are weak. Hence, geostrophic flows are likely to dominate over tides in the radiation of internal waves. The importance of wave radiation by geostrophic flows outside the Southern Ocean depends on whether it is of magnitude comparable to tidal radiation. Tidal radiation is dominated by the largest topographic scales such as mid-ocean ridges, isolated sea mountains, and continental slopes. In particular, tidal radiation has been shown to dominate in regions like the Mid-Atlantic Ridge and Hawaii (Hawaii Ocean Mixing Experiment). Less is known about wave generation by geostrophic flows in the global ocean because wave radiation is dominated by small-scale topography which is not resolved by satellite bathymetry. In addition to topographic roughness, wave radiation by geostrophic flows depends on the bottom velocity magnitude. Because of the smaller bottom velocities in regions outside the Southern Ocean, the radiation due to geostrophic flows is unlikely to be as large as in the Southern Ocean, but it might well match generation by tides. Clearly, the role of geostrophic flows in driving abyssal mixing in the global ocean is an open question and deserves more attention that previously thought.

\subsubsection{Geostrophic eddy energy budget}

The results of this thesis have implications for the overall equilibration of geostrophic eddies in the Southern Ocean. Energy dissipation in the form of internal wave radiation and breaking in regions with rough topography is a significant component of the geostrophic eddy energy budget. As shown in section 1.3.1, breaking internal waves act to spin-down the eddy by extracting both its kinetic, $E K E=\frac{1}{2} \overline{\mathbf{u}} \cdot \overline{\mathbf{u}}$, and available potential energy, $A P E=\frac{1}{2} \bar{b}^{2} / N^{2}$, according to,

$$
\partial_{t}(E K E+A P E)+\nabla \cdot \mathbf{u}(E K E+A P E)+(\bar{v} \bar{p})_{y}+(\bar{w} \bar{p})_{z} \approx-\overline{\mathbf{u}} \cdot \partial_{z} \overline{\bar{u}^{\prime} w^{\prime}}
$$

the effect of the wave buoyancy flux $\overline{b^{\prime} w^{\prime}}$ on the eddy APE is small and has been neglected. Integrating (5.4) over the volume and assuming that the energy extracted 
by the waves from the eddy balances wave energy dissipation $\epsilon$ (Chapter 2), we obtain,

$$
\partial_{t} \int(E K E+A P E) d V \approx \int \epsilon d V
$$

The characteristic time scale of geostrophic eddy spin-down by radiation and dissipation of internal waves can be roughly estimated as,

$$
T=\frac{\int(E K E+A P E) d V}{\int \epsilon d V} .
$$

At large-scales, the partitioning between EKE and APE is given by,

$$
A P E=E K E \cdot\left(1+L^{2} / L_{D}^{2}\right)
$$

where $L$ is the eddy length scale, $L_{D}$ is the Rossby radius of deformation (Pedlosky, 1986). The scale of geostrophic eddies is observed to be larger than $L_{D}$, i.e. (Ferrari and Wunsch, 2008), suggesting that the spin-down time scale must be at least as large as,

$$
T \geq 2 \frac{\int(E K E) d V}{\int \epsilon d V}=\frac{\rho_{0} \int_{0}^{H} U^{2}(z) d z}{\int_{0}^{H} \epsilon d z},
$$

where $\rho_{0}$ is a reference density and $U(z)$ is the vertical profile of geostrophic velocity.

A typical velocity profile in the Polar Front of the ACC (Fig. 3-2) varies from about $0.5 \mathrm{~m} \mathrm{~s}^{-1}$ in the ocean thermocline to $0.1 \mathrm{~m} \mathrm{~s}^{-1}$ in the deep ocean. Similar profiles are found in other ADCP sections of the Southern Ocean. With these values the eddy spin-down time $T \geq 75$ days using $E=\int_{0}^{H} \epsilon d z \approx O(10) \mathrm{mW} \mathrm{m}^{-2}$. This time scale is comparable to the time scale of eddy spin-down by bottom friction (Charney and Flierl, 1981). If the two time scales are similar it means that in regions of rough topography like Drake Passage, wave radiation is as powerful as bottom drag in extracting energy from geostrophic eddies.

The equilibration of EKE is set by a balance between the energy input by the winds and the energy dissipated through bottom friction or wave radiation and breaking (energy transfer to other latitudes is believed to be weak, e.g. Mazloff (2008)) 
Hence, the equilibration of EKE is very sensitive to the relationship between energy dissipation and total EKE. In this thesis it was shown that, for parameters characteristic of the Southern Ocean, the radiated and dissipated energy scales with $(E K E)^{5 / 4}$. This power law is intermediate between $\sim(E K E)$ and $\sim(E K E)^{3 / 2}$ characteristic of monochromatic lee wave radiation and bottom drag dissipation respectively. The power law for multichromatic topography depends on the slope of topographic spectrum at small-scales. The equilibration of the eddy field is therefore likely to depend on the characteristics of local topography, with wave radiation playing a role only in regions with rough topography.

\subsection{Future work}

\subsubsection{Theory}

There are a few limitations in this study that can be addressed in future work. First, the sensitivity of the results to the choice of bottom boundary condition in numerical simulations has not been explored extensively. 2-D simulations with free-slip and no-slip boundary condition show that wave dissipation in the latter case was reduced by about 20-30\%, while results are very similar qualitatively. It is not, however, clear whether the same applies to the 3-D problem. The effect of the boundary condition on the wave radiation and dissipation strongly depends on the low-level dynamics of mean flow-topography interaction, which is different in 2-D and 3-D.

Second, LADCP velocity data show that in the ACC fronts, which dominate wave radiation, the geostrophic flow is weakly varying in the vertical below the thermocline. Thus, as a starting point, this work considered barotropic flows and constant stratification. A natural extension would be to include vertical variations in stratification and geostrophic flow and study their effect on wave radiation and dissipation. Polzin (1999) show some evidence that even weak shears in the bottom kilometer can enhance wave breaking and energy dissipation.

Beside shear in the geostrophic flow, strong wave-wave interactions can also en- 
hance wave breaking and dissipation. In the limit of monochromatic topography, uniform mean flow and constant stratification, the vertical extent of wave dissipation region is determined by the size of upward radiating and breaking internal wave packets. The fraction of energy dissipating in the bottom kilometer is about $50 \%$ of the bottom energy radiation estimated from lee wave theory (but only $30 \%$ of the bottom energy radiation estimated from the full time-dependent wave theory). In multichromatic topography simulations, presented in Chapter 3, radiated waves are multichromatic in both vertical and horizontal wavenumbers. The vertical extent of the region where waves dissipate is the same as in monochromatic topography simulations, but the fraction of dissipated energy increases to $65 \%$ due to, probably, stronger wave-wave interaction and faster transfer of energy to smaller scales. More work needs to be done to fully understand this problem.

In this study, the mean geostrophic flow generating waves was prescribed, and represented an infinite reservoir of energy. Idealized simulations show that breaking internal waves trigger strong inertial oscillations, while the subinertial flow component does not change significantly over the 10 day period of simulations. However, on longer time scales, waves acting against the geostrophic flow and extracting energy to sustain dissipation can significantly affect its dynamics. It would be interesting to study the degree to which wave radiation extracts energy from the geostrophic eddy field and the implications of this feedback.

\subsubsection{Observations}

Besides the two LADCP/CTD sections used in Chapter 3, there are other data sets in the Southern Ocean that could be used to estimate wave radiation and dissipation or to validate results described in the thesis. The Southeast Pacific and Drake Passage sections were chosen because they are in the regions where bottom velocity, stratification, high-resolution topography data, and energy dissipation estimates were readily available, and spun the whole range from high to low mixing rates. Several other WOCE sections in the Southern Ocean (e.g. I8, I9), which include both CTD and LADCP data (Kunze et al., 2006), could be used to characterize bottom geostrophic 
flows and estimate wave radiation. In addition, there are a number of current meter moorings in Drake Passage (Nowlin et al., 1986). However, just a few of them are in the bottom kilometer. Current meter data could be used to characterize both the bottom geostrophic flow to make wave radiation estimate, and the intensity of inertial and super-inertial motions to validate theoretical prediction that inertial waves are generated by geostrophic flows with $\mathrm{Fr}^{-1} \geq 0.3$ at the bottom. In particular, it would be interesting to see whether there is an enhanced inertial frequency response in the bottom several hundred meters in regions of wave breaking and dissipation as predicted by theory, and, whether it is correlated with variations in geostrophic flow.

Finally, this work suggest that wave radiation and dissipation at the bottom of the ocean depend on topographic roughness and the magnitude of geostrophic velocity. Hence, mixing is predicted to be both spatially and temporally variable. Prompted by the results of this thesis, the recently funded Diapycnal and Isopycnal Mixing Experiment (DIMES) will attempt to estimate correlation between small-scale dissipation rates and the magnitude of the geostrophic eddy velocity and the topographic roughness. 


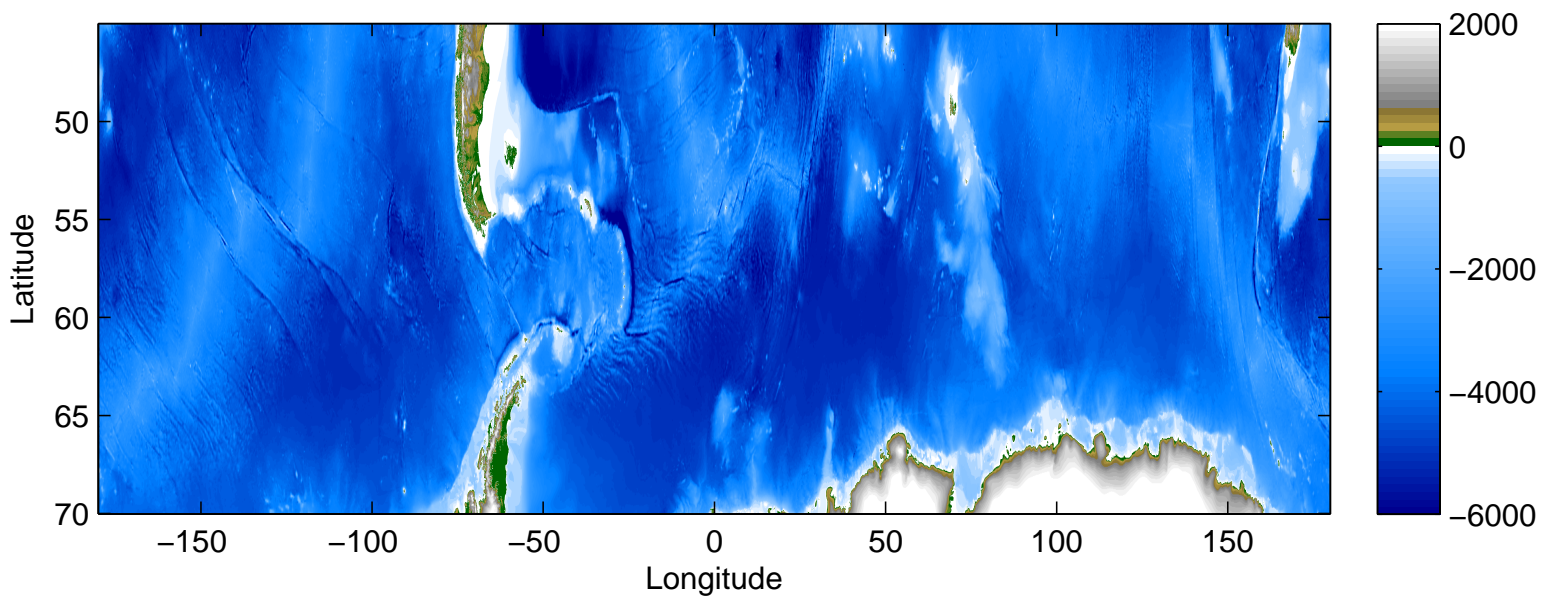

Figure 5-1: Bathymetry of the Southern Ocean (Smith and Sandwell, 1997).

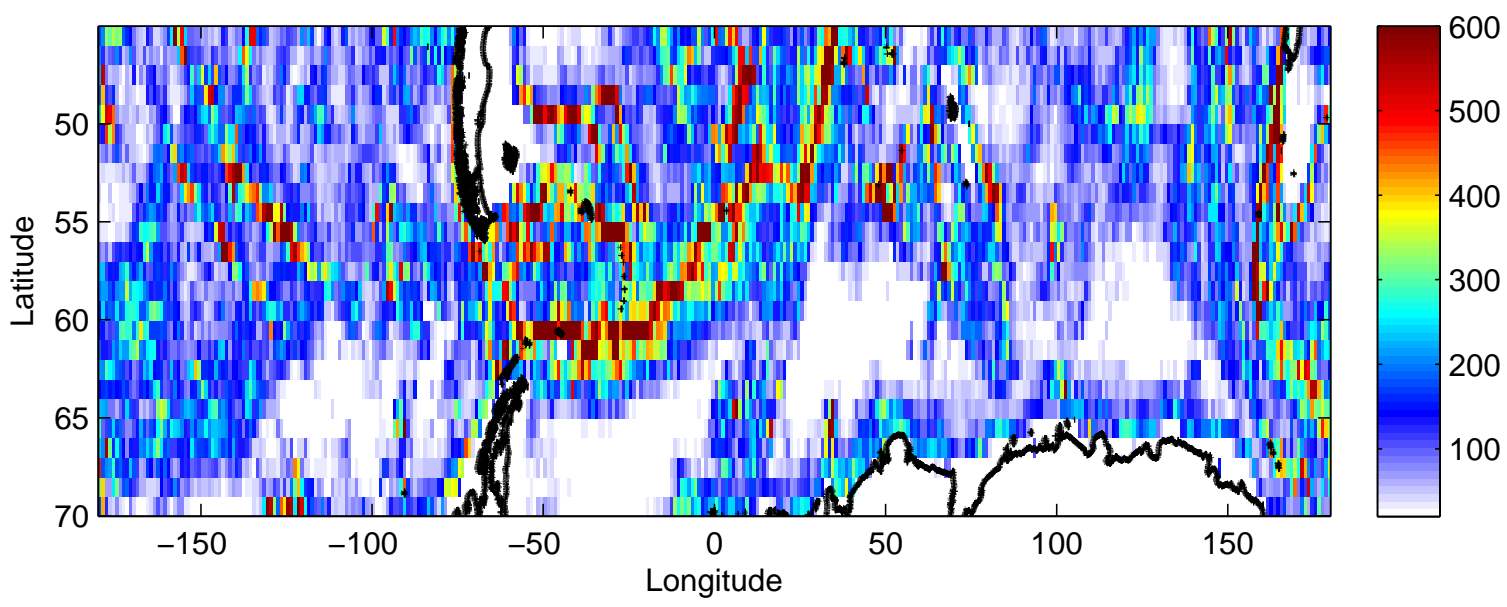

Figure 5-2: Map of the rms topographic height of the Southern Ocean computed over non-overlapping $1^{\circ} \times 1^{\circ}$ bins. 


\section{Appendix A}

\section{The equations of motion: scaling}

The expansion of the equations governing the wave-mean flow interaction is best carried out in nondimensional form. We nondimensionalize the governing equations (2.6)-(2.9) and the boundary condition (2.11) using the following scales,

$$
\begin{aligned}
& t \rightarrow f^{-1} t, \quad \mathbf{x} \rightarrow L_{W} \mathbf{x}, \quad z \rightarrow H_{W} z, \quad h_{T} \rightarrow H_{T} h, \\
& \mathbf{u} \rightarrow U_{G} \mathbf{u}, \quad w \rightarrow U_{G} \frac{H_{W}}{L_{W}} w, \quad p \rightarrow f L_{G} U_{G} p, \quad b \rightarrow \frac{f L_{G} U_{G}}{H_{G}} b,
\end{aligned}
$$

where $L_{W}, H_{W}, L_{G}$, and $H_{G}$ are the horizontal and vertical scales of the waves and subinertial flow respectively, $H_{T}$ is the topography amplitude, and $U_{G}$ is the velocity scale of the subinertial flow. Using these scale the nondimensional form of the equations is,

$$
\begin{aligned}
\mathbf{u}_{t}+R o \frac{L_{G}}{L_{W}}\left[\left(\mathbf{u} \cdot \nabla_{H}\right) \mathbf{u}+w \mathbf{u}_{z}\right]+\hat{\mathbf{z}} \times \mathbf{u} & = \\
-\frac{L_{G}}{L_{W}} \nabla_{H} p & +E k_{\nu} \frac{H_{G}^{2}}{H_{W}^{2}}\left[\delta_{W}^{2} \nabla_{H}^{2} \mathbf{u}+\mathbf{u}_{z z}\right], \\
\delta_{W}^{2} w_{t}+\delta_{W}^{2} R o \frac{L_{G}}{L_{W}}\left[\left(\mathbf{u} \cdot \nabla_{H}\right) w+w w_{z}\right] & = \\
-\frac{L_{G}}{L_{W}} p_{z}+\frac{L_{G}}{L_{W}} \frac{H_{W}}{H_{G}} b & +E k_{\nu} \delta_{W}^{2} \frac{H_{G}^{2}}{H_{W}^{2}}\left[\delta_{W}^{2} \nabla_{H}^{2} w+w_{z z}\right], \\
b_{t}+R o \frac{L_{G}}{L_{W}}\left[\left(\mathbf{u} \cdot \nabla_{H}\right) b+w b_{z}\right]+B u \frac{L_{G}}{L_{W}} \frac{H_{W}}{H_{G}} & =E k_{\kappa} \frac{H_{G}^{2}}{H_{W}^{2}}\left[\delta_{W}^{2} \nabla_{H}^{2} b+b_{z z}\right], \\
\nabla_{H} \cdot \mathbf{u}+w_{z} & =0,
\end{aligned}
$$


and the lower boundary condition becomes $\left.w\right|_{z=\epsilon h(\mathbf{x})}=\epsilon \mathbf{u} \cdot \nabla_{H} h(\mathbf{x})$. The nondimensional numbers that appear in the problem are,

\begin{tabular}{ll}
\hline \hline$R o=\frac{U_{G}}{f L_{G}}$ & Rossby number of subinertial flow \\
$\epsilon=\frac{H_{T}}{H_{W}} \sim \frac{N H_{T}}{U_{G}}$ & topography steepness parameter \\
$\delta_{W}=\frac{H_{W}}{L_{W}}$ & wave aspect ratio \\
$B u=\frac{N^{2} H_{G}^{2}}{f^{2} L_{G}^{2}}$ & Burger number of subinertial flow \\
$E k_{\nu}=\frac{\nu}{f H_{G}^{2}}, E k_{\kappa}=\frac{\kappa}{f H_{G}^{2}}$ & viscous and diffusive Ekman numbers of subinertial flow \\
\hline
\end{tabular}

Subinertial flows in the ocean have small $R o$ and evolve on scales much larger than internal waves. This scale separation is captured by imposing that the ratio between the characteristic scales of subinertial and wave flows is of order Ro,

$$
\frac{L_{W}}{L_{G}}=R o, \quad \frac{H_{W}}{H_{G}}=R o .
$$

Assuming that waves have an aspect ratio $\delta_{W}$ of $O(1)$, the Burger number of the subinertial flow is $O(1)$, and both viscous and diffusive Ekman number are such that,

$$
E k_{\nu} R o^{-2} \sim O(1), \quad E k_{\kappa} R o^{-2} \sim O(1)
$$

With this choice the nondimensional equations can be rewritten as,

$$
\begin{aligned}
\mathbf{u}_{t}+\left(\mathbf{u} \cdot \nabla_{H}\right) \mathbf{u}+w \mathbf{u}_{z}+\hat{\mathbf{z}} \times \mathbf{u} & =-R o^{-1} \nabla_{H} p+\left[\delta_{W}^{2} \nabla_{H}^{2} \mathbf{u}+\mathbf{u}_{z z}\right], \\
\delta_{W}^{2}\left[w_{t}+\left(\mathbf{u} \cdot \nabla_{H}\right) w+w w_{z}\right] & =-R o^{-1} p_{z}+b+\delta_{W}^{2}\left[\delta_{W}^{2} \nabla_{H}^{2} w+w_{z z}\right], \\
b_{t}+\left(\mathbf{u} \cdot \nabla_{H}\right) b+w b_{z}+w & =\left[\delta_{W}^{2} \nabla_{H}^{2} b+b_{z z}\right], \\
\nabla_{H} \cdot \mathbf{u}+w_{z} & =0
\end{aligned}
$$

with boundary condition,

$$
\left.w\right|_{z=\epsilon h(\mathbf{x})}=\epsilon \mathbf{u} \cdot \nabla_{H} h(\mathbf{x})
$$




\section{Appendix B}

\section{The equations of motion: multiple scale expansion}

We expand solution into a series of a small steepness parameter $\epsilon$ imposing that to the leading order there is a superposition of a geostrophic flow and an inertial oscillation,

$$
\begin{array}{r}
\mathbf{u}=\mathbf{u}^{G}\left(T_{G}, \mathbf{X}_{G}, Z_{G}\right)+\mathbf{u}^{I}\left(t ; T_{I}, \mathbf{X}_{I}, Z_{I}\right)+\epsilon \mathbf{u}^{(1)}+\epsilon^{2} \mathbf{u}^{(2)}+\epsilon^{3} \mathbf{u}^{(3)}+\ldots, \\
w=w^{G}\left(T_{G}, \mathbf{X}_{G}, Z_{G}\right)+w^{I}\left(t ; T_{I}, \mathbf{X}_{I}, Z_{I}\right)+\epsilon w^{(1)}+\epsilon^{2} w^{(2)}+\epsilon^{3} w^{(3)}+\ldots, \\
p=p^{G}\left(T_{G}, \mathbf{X}_{G}, Z_{G}\right)+\epsilon^{2} p^{I}\left(t ; T_{I}, \mathbf{X}_{I}, Z_{I}\right)+R o\left[\epsilon p^{(1)}+\epsilon^{2} p^{(2)}+\epsilon^{3} p^{(3)}+\ldots\right], \\
b=b^{G}\left(T_{G}, \mathbf{X}_{G}, Z_{G}\right)+\epsilon^{3} b^{I}\left(t ; T_{I}, \mathbf{X}_{I}, Z_{I}\right)+\epsilon b^{(1)}+\epsilon^{2} b^{(2)}+\epsilon^{3} b^{(3)}+\ldots,
\end{array}
$$

where $\mathbf{u}^{G}$ is the geostrophic flow evolving on a slow time scale $T_{G}$ and large spatial scales $\mathbf{X}_{G}$ and $Z_{G}, \mathbf{u}^{I}$ is an inertial oscillation which oscillates at frequency $f$ on a fast time scale $t$ and can also evolve on a slow time scale $T_{I}$ and large spatial scales $\mathbf{X}_{I}$ and $Z_{I}$, and $\mathbf{u}^{(n)}$ are the higher order motions that depend on all scales of the problem.

We make the following choice of scales for geostrophic flow component which is consistent with the scaling arguments used to nondimensionalize the equations,

$$
T_{G}=\text { Rot }, \quad \mathbf{X}_{G}=\text { Rox }, \quad Z_{G}=\text { Roz } .
$$


Inertial oscillations, in this problem, result from geostrophic adjustment of subinertial flow and are forced by vertical divergence of internal wave momentum flux. Therefore, we assume that the horizontal scale of inertial oscillations is of the same order as the scale of subinertial flow and vertical scale is set by the scale of internal wave momentum flux, which is assumed to be $O\left(\epsilon^{-1}\right)$ larger than the wave scale,

$$
T_{I}=\epsilon^{3} t, \quad \mathbf{X}_{I}=R o \mathbf{x}, \quad Z_{I}=\epsilon z .
$$

This choice of scales allows both time and space scale separation between subinertial flow, inertial oscillations and higher order motions. Rossby number, $R o$, is assumed to be smaller than $\epsilon^{3}$ to separate internal wave dynamics from quasigeostrophic motion dynamics.

Applying flow decomposition to the equations (A.3)-(A.6) with the lower boundary condition (A.7) and collecting $O\left(\epsilon^{0}\right)$ terms, we get a set of equations describing the evolution of the leading order flow,

$$
\begin{aligned}
\mathbf{u}_{t}^{I}+\hat{\mathbf{z}} \times\left(\mathbf{u}^{G}+\mathbf{u}^{I}\right) & =-\nabla_{\mathbf{x}_{G}} p^{G}, \\
\delta_{W}^{2} w_{t}^{I} & =-p_{Z_{G}}^{G}+b^{G}, \\
w^{G}+w^{I} & =0, \\
\left.\left(w^{G}+w^{I}\right)\right|_{z=0} & =0 .
\end{aligned}
$$

Taking an average of this set of equations over the fast time scale $t$, we can separate geostrophic flow evolving on subinertial times $T_{G}=$ Rot only,

$$
\hat{\mathbf{z}} \times \mathbf{u}^{G}=-\nabla_{\mathbf{x}_{G}} p^{G}, \quad 0=-p_{Z_{G}}^{G}+b^{G}, \quad w^{G}=0,
$$

from an inertial oscillation evolving on the fast time scale $t$,

$$
\mathbf{u}_{t}^{I}+\hat{\mathbf{z}} \times \mathbf{u}^{I}=0, \quad w^{I}=0 .
$$


Next, collecting $O\left(\epsilon^{1}\right)$ terms, we obtain,

$$
\begin{aligned}
\mathbf{u}_{t}^{(1)}+\left(\mathbf{u}^{G}+\mathbf{u}^{I}\right) \cdot \nabla_{\mathbf{x}} \mathbf{u}^{(1)}+\hat{\mathbf{z}} \times \mathbf{u}^{(1)} & \left.=-\nabla_{\mathbf{x}} p^{(1)}+\left[\delta_{W}^{2} \nabla_{H}^{2} \mathbf{u}^{(1)}+\mathbf{u}_{z z}^{(1)}\right],\left(\mathbf{u}^{I}\right) \cdot \nabla_{\mathbf{x}} w^{(1)}\right] \\
\delta_{W}^{2}\left[w_{t}^{(1)}+\left(\mathbf{u}^{G}+\mathbf{u}^{(1)}+b^{(1)}\right.\right. & +\delta_{W}^{2}\left[\delta_{W}^{2} \nabla_{H}^{2} w^{(1)}+w_{z z}^{(1)}\right] \\
b_{t}^{(1)}+\left(\mathbf{u}^{G}+\mathbf{u}^{I}\right) \cdot \nabla_{\mathbf{x}} b^{(1)}+w^{(1)} & =\left[\delta_{W}^{2} \nabla_{H}^{2} b^{(1)}+b_{z z}^{(1)}\right] \\
\nabla_{\mathbf{x}} \cdot \mathbf{u}^{(1)}+w_{z}^{(1)} & =0 \\
\left.w^{(1)}\right|_{z=0} & =\left(\mathbf{u}_{G}+\mathbf{u}_{I}\right) \cdot \nabla_{\mathbf{x}} h
\end{aligned}
$$

This set of equations describes the evolution of $O\left(\epsilon^{1}\right)$ motions generated by geostrophic flow $\mathbf{u}_{G}$ with an inertial oscillation $\mathbf{u}_{I}$ interacting with bottom topography. To capture evolution of inertial oscillation on subinertial time scale $T_{I}=\epsilon^{3} t$ we need to expand equations to higher order. Averaging $O\left(\epsilon^{1}\right)$ equations over the small spatial scales filters out internal wave motions and leaves an $O\left(\epsilon^{1}\right)$ inertial ocsillations,

$$
\overline{\mathbf{u}_{t}^{(1)}}+f \hat{\mathbf{z}} \times \overline{\mathbf{u}^{(1)}}=0, \quad \overline{w^{(1)}}=0 .
$$

Now, collecting $O\left(\epsilon^{2}\right)$ terms, we obtain,

$$
\begin{aligned}
\mathbf{u}_{t}^{(2)}+\left(\mathbf{u}^{G}+\mathbf{u}^{I}\right) \cdot \nabla_{\mathbf{x}} \mathbf{u}^{(2)}+f \hat{\mathbf{z}} \times \mathbf{u}^{(2)} & =-\nabla_{\mathbf{x}} p^{(2)} \\
-w^{(1)} \mathbf{u}_{Z_{I}}^{I} & -\mathbf{u}^{(1)} \nabla_{\mathbf{x}} \mathbf{u}^{(1)}-w^{(1)} \mathbf{u}_{z}^{(1)}, \\
w_{t}^{(2)}+\left(\mathbf{u}^{G}+\mathbf{u}^{I}\right) \cdot \nabla_{\mathbf{x}} w^{(2)} & =-p_{z}^{(2)}+b^{(2)}-p_{Z_{I}}^{(1)} \\
& -\mathbf{u}^{(1)} \nabla_{\mathbf{x}} w^{(1)}-w^{(1)} w_{z}^{(1)}, \\
b_{t}^{(2)}+\left(\mathbf{u}^{G}+\mathbf{u}^{I}\right) \cdot \nabla_{\mathbf{x}} b^{(2)}+w^{(2)} N^{2} & =-\mathbf{u}^{(1)} \nabla_{\mathbf{x}} b^{(1)}-w^{(1)} b_{z}^{(1)}, \\
\nabla_{\mathbf{x}} \cdot \mathbf{u}^{(2)}+w_{z}^{(2)} & =-w_{Z_{I}}^{(1)} . \\
\left.w^{(2)}\right|_{z=0} & =\mathbf{u}^{(1)} \cdot \nabla_{\mathbf{x}} h-h w_{z}^{(1)},
\end{aligned}
$$

Equivalently, averaging over small spatial scales we obtain equations for the $O\left(\epsilon^{2}\right)$ 
inertial oscillations,

$$
\overline{\mathbf{u}_{t}^{(2)}}+f \hat{\mathbf{z}} \times \overline{\mathbf{u}^{(2)}}=0, \quad \overline{w^{(2)}}=0 .
$$

Finally, collecting $O\left(\epsilon^{3}\right)$ terms, we get,

$$
\begin{aligned}
\mathbf{u}_{t}^{(3)}+\left(\mathbf{u}^{G}+\mathbf{u}^{I}\right) \cdot \nabla_{\mathbf{x}} \mathbf{u}^{(3)}+f \hat{\mathbf{z}} \times \mathbf{u}^{(3)} & =-\nabla_{\mathbf{x}} p^{(3)}-\mathbf{u}_{T_{I}}^{I}-w^{(2)} \mathbf{u}_{Z_{I}}^{I}- \\
-\mathbf{u}^{(1)} \nabla_{\mathbf{x}} \mathbf{u}^{(2)}-w^{(1)} \mathbf{u}_{z}^{(2)}-\mathbf{u}^{(2)} \nabla_{\mathbf{x}} \mathbf{u}^{(1)} & -w^{(2)} \mathbf{u}_{z}^{(1)}-w^{(1)} \mathbf{u}_{Z_{I}}^{(1)} \\
w_{t}^{(3)}+\left(\mathbf{u}^{G}+\mathbf{u}^{I}\right) \cdot \nabla_{\mathbf{x}} w^{(3)} & =-p_{z}^{(3)}+b^{(3)}-p_{Z_{I}}^{I}+b^{I}-p_{Z_{I}}^{(2)}- \\
-\mathbf{u}^{(1)} \nabla_{\mathbf{x}} w^{(2)}-w^{(1)} w_{z}^{(2)}-\mathbf{u}^{(2)} \nabla_{\mathbf{x}} w^{(1)} & -w^{(2)} w_{z}^{(1)}-w^{(1)} w_{Z_{I}}^{(1)} \\
b_{t}^{(3)}+\left(\mathbf{u}^{G}+\mathbf{u}^{I}\right) \cdot \nabla_{\mathbf{x}} b^{(3)}+w^{(3)} N^{2} & =-b_{t}^{I}-\mathbf{u}^{(1)} \nabla_{\mathbf{x}} b^{(2)}- \\
-w^{(1)} b_{z}^{(2)}-\mathbf{u}^{(2)} \nabla_{\mathbf{x}} b^{(1)} & -w^{(2)} b_{z}^{(1)}-w^{(1)} b_{Z_{I}}^{(1)} \\
\nabla_{\mathbf{x}} \cdot \mathbf{u}^{(3)}+w_{z}^{(3)} & =-w_{Z_{I}}^{(2)} \\
\left.w^{(3)}\right|_{z=0}=\mathbf{u}^{(2)} \cdot \nabla_{\mathbf{x}} h & +h \mathbf{u}_{z}^{(1)} \cdot \nabla_{\mathbf{x}} h+ \\
h \mathbf{u}_{Z_{I}}^{I} \cdot \nabla_{\mathbf{x}} h-h w_{Z_{I}}^{(1)} & -h w_{z}^{(2)}-\frac{1}{2} h^{2} w_{z z}^{(1)}
\end{aligned}
$$

Averaging over the small spatial scales, we obtain,

$$
\begin{aligned}
\overline{\mathbf{u}_{t}^{(3)}}+f \hat{\mathbf{z}} \times \overline{\mathbf{u}^{(3)}} & =-\mathbf{u}_{T_{I}}^{I}-\partial_{Z_{I}} \overline{w^{(1)} \mathbf{u}^{(1)}}, \\
0 & =-\partial_{Z_{I}} \overline{w^{(1)} w^{(1)}}-P_{Z_{I}}^{I}+b^{I}, \\
b_{t}^{I} & =-\partial_{Z_{I}} \overline{w^{(1)} b^{(1)}}
\end{aligned}
$$

where over-bar is an average over $\mathbf{x}$, and $z$ scales. 


\section{Bibliography}

Adcroft, A., C. Hill, and J. Marshall, 1997: Representation of topography by shaved cells in a height coordinate ocean model. Mon. Wea. Rev., 125, 2293-2315.

Balmforth, N., G. Ierley, and W. Young, 2002: Tidal conversion by nearly critical topography. J. Phys. Oceanogr., 32, 2900-2914.

Bell, T., 1975a: Lee waves in stratified flows with simple harmonic time dependence. J. Fluid Mech., 67, 705-722.

Bell, T., 1975b: Topographically generated internal waves in the open ocean. $J$. Geophys. Res., 80, 320-327.

Bretherton, F., 1969: Momentum transport by gravity waves. Quart. J. Roy. Meteor. Soc., 95, 213-243.

Broutman, D. and W. Young, 1986: On the interaction of small-scale oceanic internal waves with near-inertial waves. J. Fluid Mech., 166, 341-358.

Charney, J. and G. Flierl, 1981: Oceanic analogues of large-scale atmospheric motions. Evolution of Physical Oceanography, B. A. Warren and C. Wunsch, Eds., MIT Press, 504-548.

Dickson, R., 1990: Flow statistics from long-term current- meter moorings. the global data-set in january 1989. World Meteorological Office Technical Document WMO/TD - N337.

Egbert, G., A. Bennett, and M. Foreman, 1994: Topex/poseidon tides estimated using global inverse model. J. Geophys. Res., 99, 24821-24852.

Egbert, G. and S. Erofeeva, 2002: Efficient inverse modeling of barotropic ocean tides. J. Atmos. Oceanic Technol., 19, 183-206.

Ferrari, R. and C. Wunsch, 2008: Ocean circulation kinetic energy - reservoirs, sources and sinks. AFM in press.

Ganachaud, A. and C. Wunsch, 2000: Improved estimates of global ocean circulation, heat transport and mixing from hydrographic data. Nature, 408, 453-456.

Garrett, C. and E. Kunze, 2007: Internal tide generation in the deep ocean. Annu. Rev. Fluid Mech., 39, 57-87. 
Garrett, C. and L. St.-Laurent, 2002: Aspects of deep ocean mixing. J. Oceanogr., $58,11-24$.

Goff, J. and T. Jordan, 1988: Stochastic modeling of seafloor morphology: Inversion of sea beam data for second-order statistics. J. Geophys. Res., 93, 13 589-13608.

Gregg, M., 1989: Scaling turbulent dissipation in the thermocline. J. Geophys. Res., 94, 9686-9698.

Heywood, K. and D. Stevens, 2000: Albatross cruise report. UEA Cruise Report series 6, UEA publications, UEA, Norwich, UK.

Ito, T. and J. Marshall, 2008: Control of lower limb circulation in the southern ocean by diapycnal mixing and mesoscale eddy transfer. submitted to JPO.

Khatiwala, S., 2003: Generation of internal tides in an ocean of finite depth: analytical and numerical calculations. Deep-Sea Research I, 50, 3-21.

Kunze, E., E. Firing, J. Hummon, T. Chereskin, and A. M. Thurnherr, 2006: Global abyssal mixing inferred from lowered adcp shear and ctd strain profiles. J. Phys. Oceanogr., 36, 1553-1576.

Ledwell, J., E. Montgomery, K. Polzin, L. St.-Laurent, R. Schmitt, and J. Toole, 2000: Evidence for enhanced mixing over rough topography. Nature, 403, 179-182.

Ledwell, J., A. Watson, and C. Law, 1998: Mixing of a tracer released in the pycnocline. J. Geophys. Res., 103, 21 499-21529.

Legg, S. and K. Huijts, 2006: Preliminary simulations of internal waves and mixing generated by finite amplitude tidal flow over isolated topography. Deep-Sea Research II, 53, 140-156.

Llewellyn-Smith, S. and W. Young, 2002: Conversion of the barotropic tide. J. Phys. Oceanogr., 32, 1554-1566.

Llewellyn-Smith, S. and W. Young, 2003: Tidal conversion at a very steep ridge. $J$. Fluid Mech., 495, 175-191.

Long, R., 1953: Some aspects of the flow of stratified fluids. Tellus, 5, 42-58.

Marshall, J., A. Adcroft, C. Hill, L. Perelman, and C. Heisey, 1997: A finite-volume, incompressible navier-stokes model for studies of the ocean on parallel computers. J. Geophys. Res., 102, 5753-5766.

Marshall, J. and T. Radko, 2003: Residual mean solutions for the antarctic circumpolar current and its associated overturning circulation. J. Phys. Oceanogr., 33, 2341-2354. 
Mazloff, M., 2008: The southern ocean meridional overturning circulation as diagnosed from an eddy permitting state estimate. Ph.D. thesis, Massachusetts Institute of Technology, 108 pp.

McComas, C. and F. Bretherton, 1977: Resonant interaction of oceanic internal waves. J. Geophys. Res., 82, 1397-1412.

Munk, W., 1981: Internal waves and small-scale processes. Evolution of physical oceanography, B. A. Warren and C. Wunsch, Eds., MIT Press, Cambridge, 264291.

Naveira-Garabato, A., K. Heywood, and D. Stevens, 2002: Modification and pathways of southern ocean deep waters in the scotia sea. Deep-Sea Research, 49, 681-705.

Naveira-Garabato, A., K. Polzin, B. King, K. Heywood, and M. Visbeck, 2004: Widespread intense turbulent mixing in the southern ocean. Science, 303, 210213.

Naveira-Garabato, A., D. Stevens, and K. Heywood, 2003: Water mass conversion, fluxes, and mixing in the scotia sea diagnosed by an inverse model. J. Phys. Oceanogr., 33, 2565-2587.

Nowlin, W., J. Bottero, and R. Pillsbury, 1986: Observations of internal and nearinertial oscillations at drake passage. J. Phys. Oceanogr., 16, 87-108.

Nycander, J., 2005: Generation of internal waves in the deep ocean by tides. J. Geophys. Res., 110, C10028, doi:10.1029/2004JC002487.

Olbers, D., D. Borowski, C. Volker, and J. Wolff, 2004: The dynamical balance, transport and circulation of the antarctic circumpolar current. Antarctic Science, 16, 439-470.

Osborn, T., 1980: Estimates of the local rate of vertical diffusion from dissipation measurements. J. Phys. Oceanogr., 10, 83-89.

Padman, L., S. Howard, and R. Muench, 2006: Internal tide generation along the south scotia ridge. Deep-Sea Research II, 53, 157-171.

Pedlosky, J., 1986: Geophysical fluid dynamics. Springer-Verlag.

Polzin, K., J. Toole, J. Ledwell, and R. Schmitt, 1997: Spatial variability of turbulent mixing in the abyssal ocean. Science, 276, 93-96.

Polzin, K. L., 2004: Idealized solutions for the energy balance of the finescale internal wavefield. J. Phys. Oceanogr., 34, 231-246.

Polzin, K. L., 2008: Internal wave generation and scattering from rough topography. submitted. 
Polzin, K. L. and E. Firing, 1997: Estimates of diapycnal mixing using ladep and ctd data from i8s. WOCE International Newsletter, 29, 29-42.

Polzin, K. L., J. M. Toole, and R. W. Schmitt, 1995: Finescale parameterizations of turbulent dissipation. J. Phys. Oceanogr., 25, 306-328.

Rintoul, S., C. Hughes, and D. Olbers, 2001: The antarctic circumpolar current system. Ocean Circulation and Climate, J. C. G. Siedler and J. Gould, Eds., Academic Press, 271-302.

Sen, A., R. Scott, and B. K. Arbic, 2008: Global energy dissipation rate of deep-ocean low-frequency flows by quadratic bottom boundary layer drag: Computations from current-meter data. Geophys. Res. Lett., 35, L09606, doi:10.1029/2008GL033407.

Sloyan, B. and S. Rintoul, 2001: The southern ocean limb of the global deep overturning circulation. J. Phys. Oceanogr., 31, 143-173.

Smith, W. and D. Sandwell, 1997: Global seafloor topography from satellite altimetry and ship depth soundings. Science, 277, 1957-1962.

Speer, K., S. Rintoul, and B. Sloyan, 2000: The diabatic deacon cell. J. Phys. Oceanogr., 30, 3212-3222.

St.-Laurent, L. and C. Garrett, 2002: The role of internal tides in mixing the deep ocean. J. Phys. Oceanogr., 32, 2882-2899.

St.-Laurent, L., J. Toole, and R. Schmitt, 2001: Buoyancy forcing by turbulence above rough topography in the abyssal brazil basin. J. Phys. Oceanogr., 31, 3476-3495.

Stammer, D., 1997: Global characteristics of ocean variability estimated from regional topex/poseidon altimeter measurements. J. Phys. Oceanogr., 27, 1743-1769.

von Storch, J., H. Sasaki, and J. Marotzke, 2007: Wind-driven power input to the deep ocean: An estimate using a $1 / 10^{\circ}$ general circulation model. J. Phys. Oceanogr., 37, 657-672.

Wunsch, C., 1997: The vertical partition of oceanic horizontal kinetic energy. J. Phys. Oceanogr., 27, 1770-1794.

Wunsch, C., 1998: The work done by the wind on the oceanic general circulation. J. Phys. Oceanogr., 28, 2331-2339.

Wunsch, C. and R. Ferrari, 2004: Vertical mixing, energy, and the general circulation of the ocean. Agric. For. Meteor., 36, 281-314.

Wurtele, M., R. Sharman, and A. Datta, 1996: Atmospheric lee waves. Annu. Rev. Fluid Mech., 28, 429-476. 